\title{
ESTIMATED USE OF WATER IN THE UNITED STATES IN 2000
}

By Susan S. Hutson, Nancy L. Barber, Joan F. Kenny, Kristin S. Linsey, Deborah S. Lumia, and Molly A. Maupin

U.S. Geological Survey Circular 1268 


\section{U.S. Department of the Interior \\ Gale A. Norton, Secretary}

\section{U.S. Geological Survey \\ Charles G. Groat, Director}

\section{U.S. Geological Survey, Reston, Virginia: 2004}

For sale by U.S. Geological Survey, Information Services

Box 25286, Denver Federal Center

Denver, CO 80225

For more information about the USGS and its products:

Telephone: 1-888-ASK-USGS

World Wide Web: http://www.usgs.gov/

Any use of trade, product, or firm names in this publication is for descriptive purposes only and does not imply endorsement by the U.S. Government.

Although this report is in the public domain, permission must be secured from the individual copyright owners to reproduce any copyrighted materials contained within this report.

Suggested citation:

Hutson, S.S., Barber, N.L., Kenny, J.F., Linsey, K.S., Lumia, D.S., and Maupin, M.A., 2004, Estimated use

of water in the United States in 2000: Reston, Va., U.S. Geological Survey Circular 1268, 46 p.

\section{Library of Congress Cataloging-in-Publication Data}

Estimated use of water in the United States in 2000 / by Susan S. Hutson ... [et al.].

p. cm. -- (U.S. Geological Survey circular ; 1268)

Includes bibliographic references.

ISBN 0-607-97818-X (pbk.)

1. Water consumption--United States. I. Hutson, Susan S. II. Series.

TD223.E835 2004

$333.91^{\prime} 13^{\prime} 0973090511--d c 22$ 


\section{Foreword}

ince 1950, the U.S. Geological Survey (USGS) has compiled data at five-year intervals on amounts of water used in homes, businesses, industries, and on farms throughout the United States, and has described how that use has changed with time. Water-use data, combined with other USGS information, have facilitated a unique understanding of the effects of human activity on the Nation's water resources. As water availability continues to emerge as an important issue in the 21 st century, the need for consistent, long-term wateruse data will increase to support wise use of this essential natural resource.

This Circular documents water use in 2000 and identifies important changes in water use that have occurred over the past 50 years. The early part of this history (1950 to 1980) showed a steady increase in water use. During this time, the expectation was that as population increased, so would water use. Contrary to expectation, reported water withdrawals declined in 1985 and have remained relatively stable since then. Changes in technology, in State and Federal laws, and in economic factors, along with increased awareness of the need for water conservation, have resulted in more efficient use of the water from the Nation's rivers, lakes, reservoirs, and aquifers.
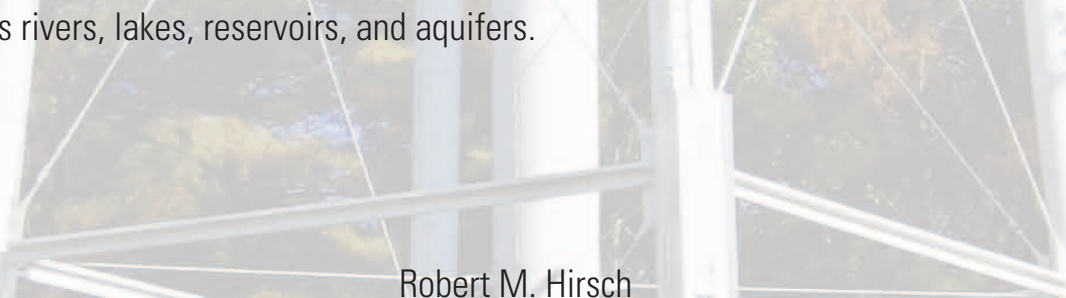

Associate Director for Water

More detailed water-use information is available on the Internet at http://water.usgs.gov/watuse 


\section{Contents}

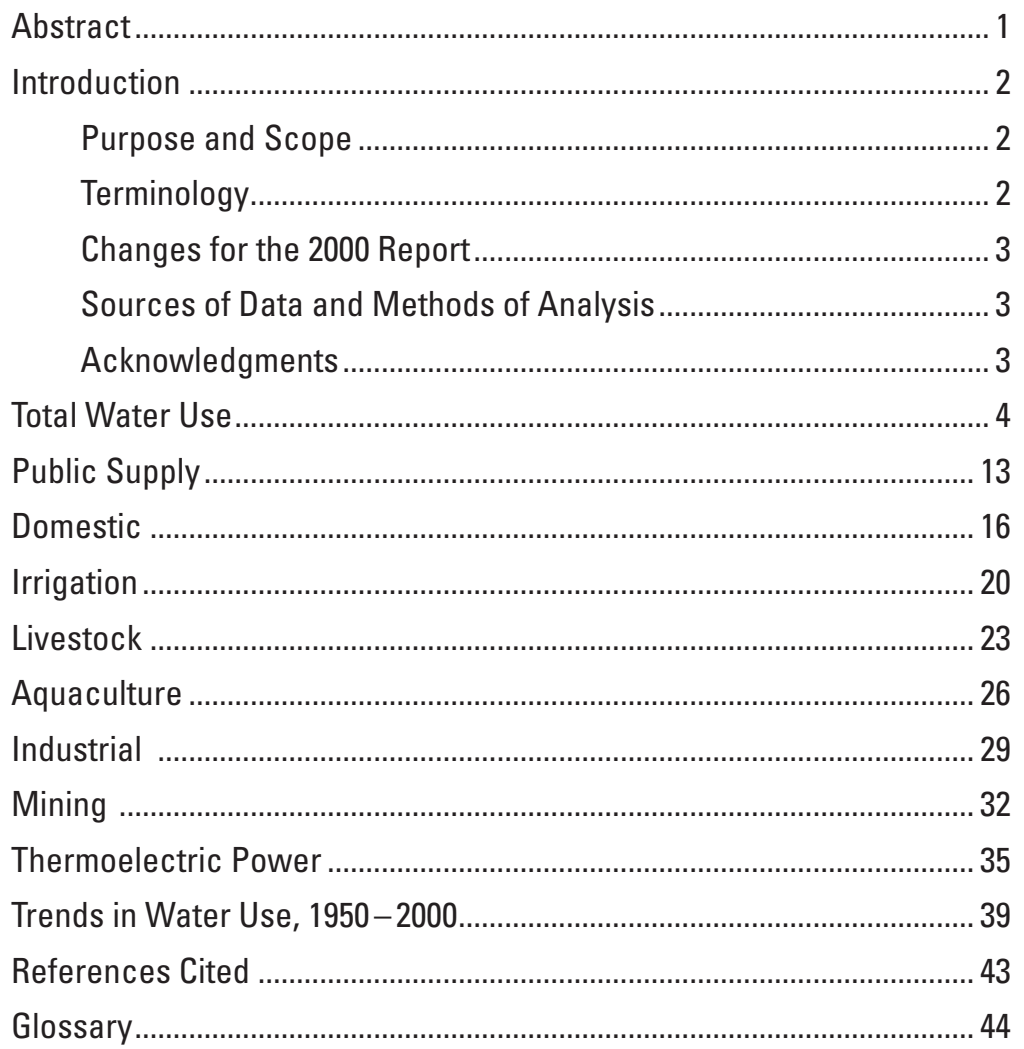




\section{Conversion Factors}

\begin{tabular}{lll} 
Multiply & By & To obtain \\
\hline acre & Area & \\
\hline & 43,560 & square foot $\left(\mathrm{ft}^{2}\right)$ \\
& 4,047 & square meter $\left(\mathrm{m}^{2}\right)$ \\
\hline gallon per day (gal/d) & 0.001562 & square mile $\left(\mathrm{mi}^{2}\right)$ \\
\hline million gallons per day (Mgal/d) & 3.785 & liter per day \\
& 1.121 & thousand acre-feet per year \\
& 0.001547 & thousand cubic feet per second \\
& 0.6944 & thousand gallons per minute \\
billion gallons per day (Bgal/d) & 0.003785 & million cubic meters per day \\
thousand acre-feet day per year & 1.3815 & million cubic meters per year \\
& 1.3815 & billion cubic meters per year \\
& 0.8921 & million gallons per day \\
& 0.001380 & thousand cubic feet per second \\
& 0.6195 & thousand gallons per minute \\
& 0.003377 & million cubic meters per day
\end{tabular}

Some water relations in inch-pound units are shown below

1 gallon (gal)

1 million gallons (Mgal)

1 cubic foot $\left(\mathrm{ft}^{3}\right)$

1 acre-foot (acre-ft)

1 inch of rain
8.34 pounds

3.07 acre-feet

62.4 pounds

7.48 gallons

325,851 gallons

43,450 cubic feet

17.4 million gallons per square mile

27,200 gallons per acre

100 tons per acre 


\title{
ESTIMATED USE OF WATER IN THE UNITED STATES IN 2000
}

\author{
By Susan S. Hutson, Nancy L. Barber, Joan F. Kenny, Kristin S. Linsey, \\ Deborah S. Lumia, and Molly A. Maupin
}

\begin{abstract}
Estimates of water use in the United States indicate that about 408 billion gallons per day (one thousand million gallons per day, abbreviated Bgal/d) were withdrawn for all uses during 2000. This total has varied less than 3 percent since 1985 as withdrawals have stabilized for the two largest uses - thermoelectric power and irrigation. Fresh groundwater withdrawals $(83.3 \mathrm{Bgal} / \mathrm{d})$ during 2000 were 14 percent more than during 1985 . Fresh surface-water withdrawals for 2000 were $262 \mathrm{Bgal} / \mathrm{d}$, varying less than 2 percent since 1985 .

About $195 \mathrm{Bgal} / \mathrm{d}$, or 48 percent of all freshwater and saline-water withdrawals for 2000 , were used for thermoelectric power. Most of this water was derived from surface water and used for once-through cooling at power plants. About 52 percent of fresh surface-water withdrawals and about 96 percent of saline-water withdrawals were for thermoelectric-power use. Withdrawals for thermoelectric power have been relatively stable since 1985 .

Irrigation remained the largest use of freshwater in the United States and totaled $137 \mathrm{Bgal} / \mathrm{d}$ for 2000. Since 1950, irrigation has accounted for about 65 percent of total water withdrawals, excluding those for thermoelectric power. Historically, more surface water than ground water has been used for irrigation. However, the percentage of total irrigation withdrawals from ground water has continued to increase, from 23 percent in 1950 to 42 percent in 2000. Total irrigation withdrawals were 2 percent more for 2000 than for 1995 , because of a 16 percent increase in ground-water withdrawals and a small decrease in surface-water withdrawals. Irrigated acreage more than doubled between 1950 and 1980, then
\end{abstract}

remained constant before increasing nearly 7 percent between 1995 and 2000. The number of acres irrigated with sprinkler and microirrigation systems has continued to increase and now comprises more than one-half the total irrigated acreage.

Public-supply withdrawals were more than $43 \mathrm{Bgal} / \mathrm{d}$ for 2000. Public-supply withdrawals during 1950 were $14 \mathrm{Bgal} / \mathrm{d}$. During 2000, about 85 percent of the population in the United States obtained drinking water from public suppliers, compared to 62 percent during 1950. Surface water provided 63 percent of the total during 2000, whereas surface water provided 74 percent during 1950.

Self-supplied industrial withdrawals totaled nearly $20 \mathrm{Bgal} / \mathrm{d}$ in 2000, or 12 percent less than in 1995. Compared to 1985 , industrial self-supplied withdrawals declined by 24 percent. Estimates of industrial water use in the United States were largest during the years from 1965 to 1980, but during 2000, estimates were at the lowest level since reporting began in 1950. Combined withdrawals for self-supplied domestic, livestock, aquaculture, and mining were less than $13 \mathrm{Bgal} / \mathrm{d}$ for 2000 , and represented about 3 percent of total withdrawals.

California, Texas, and Florida accounted for one-fourth of all water withdrawals for 2000. States with the largest surface-water withdrawals were California, which had large withdrawals for irrigation and thermoelectric power, and Texas, which had large withdrawals for thermoelectric power. States with the largest ground-water withdrawals were California, Texas, and Nebraska, all of which had large withdrawals for irrigation. 


\section{INTRODUCTION}

This report, "Estimated use of water in the United States in 2000," marks 50 years of water-use data compiled by the U.S. Geological Survey (USGS). Data on water withdrawals by State, source of water, and category of use have been compiled at 5-year intervals since 1950. Data from this Circular and other USGS water-use Circulars can be used along with information on the availability of ground water and surface water to assess water-resource management needs in the face of changing demands for water. Reliable water-use data are essential to many organizations and individuals in support of research and policy decisions.

Since 1950, water supplies and their uses have been affected by population growth, economic trends, legal decisions, and periodic droughts. In response to constraints on water supplies, communities have expanded their watersupply infrastructures or instituted water-conservation measures, farmers have changed crops or agricultural practices, and industries have reused or reclaimed process water. Population changes affecting water use during the time period from 1950 to 2000 include an overall growth of 90 percent, with a shift in the population of the United States from rural areas to urban areas and a continuing shift of the mean geographic center of population west and south (Hobbs and Stoops, 2002). In some geographic areas, the availability of water and improved technology have resulted in increases in irrigated acreage and irrigation water use. In other areas, increased costs and reduced water availability have led to more efficient irrigation practices and a reduction in irrigation water use. Changes in production, technology, and economic conditions have affected industrial water use. Periodic droughts have drawn attention to limits in the reliability of local and regional water supplies and influenced short-term water use for all users.

Climatic fluctuations affect water withdrawals, particularly for irrigation, power generation, public supply, and self-supplied domestic water use. However, effects of extremes in temperature and precipitation often are difficult to isolate from other factors that affect water use; thus, climatic effects cannot be identified readily based on the aggregated data contained in this report.

The year 2000 was one of climatic extremes. Weather in the Midwest and Northeast was characterized by prolonged periods of cooler and wetter than normal conditions. During the summer months (June-August), precipitation was above average in 15 States throughout this region. The South and West experienced severe drought as a result of belownormal precipitation and above-normal temperatures. Alabama, Florida, Georgia, Louisiana, and Mississippi had the driest May-October period on record during 2000. Streams and reservoirs dropped to record low levels, and some cities imposed drought restrictions. The driest July-September period was recorded in Arkansas, Kansas, Louisiana, Mississippi, Oklahoma, and Texas. Much of the western United
States also was in severe drought-including Arizona, Colorado, Idaho, Montana, New Mexico, Utah, and Wyoming. By August 2000, 36 percent of the United States was in severe to extreme drought, leading to widespread wildfires and other drought-related damages (National Oceanic and Atmospheric Administration, 2001; U.S. Department of Agriculture, 2000).

\section{Purpose and Scope}

This report presents consistent and current water-use estimates by source and by State for the States, the District of Columbia, Puerto Rico, and the U.S. Virgin Islands (referred to in the text as States or United States for brevity). The USGS has compiled similar national estimates every 5 years since 1950 (MacKichan, 1951, 1957; MacKichan and Kammerer, 1961; Murray, 1968; Murray and Reeves, 1972, 1977; and Solley and others, 1983, 1988, 1993, 1998). This series of water-use reports serves as one of the few sources of information about regional or national trends in water withdrawals. The report provides information on eight categories of water use - public supply, domestic, irrigation, livestock, aquaculture, industrial, mining, and thermoelectric power. This report contains a section on total water use for 2000 , followed by more detailed discussions for each category. The final section presents a discussion on trends in water use from 1950 to 2000.

\section{Terminology}

The terms and units used in this report are similar to those used in previous USGS water-use Circulars and are defined in the Glossary (at the end of the report). For 2000, water use was defined as water withdrawals rather than as water withdrawals plus deliveries from public supplies. Saline water, defined as water that contains 1,000 milligrams per liter or more of dissolved solids, was tabulated for the industrial, mining, and thermoelectric-power categories. All publicsupply, self-supplied domestic, irrigation, and livestock withdrawals were considered freshwater in this report. For aquaculture, only freshwater withdrawals were reported for 2000.

Annual water-use data are expressed in terms of million gallons per day (abbreviated as Mgal/d) and thousand acrefeet per year in this report. The term billion gallons per day (one-thousand million gallons per day, abbreviated as Bgal/d) is used in the Abstract and Trends sections of this report to more simply express large numbers. Units of million gallons per day or billion gallons per day are used to indicate an average daily rate of usage, and do not represent actual daily rates. For example, irrigation water is applied only part of each year and at variable rates; therefore, the actual rate of application at any given time during the growing season would be more than the average daily rate expressed as million gallons per day or as billion gallons per day.

The water-use data in this report are rounded to three significant figures. All values are rounded independently; therefore, the sums of individual rounded numbers may not equal 
the totals. The percentage changes discussed in the text are calculated from the unrounded data and expressed as integers. All population data are rounded to three significant figures.

\section{Changes for the 2000 Report}

The number of reported categories and the data elements collected were reduced for 2000, and some States collected data for only certain core categories. Emphasis was placed on ensuring the quality of data that were collected, rather than attempting to provide all categories and data elements on a national scale. Additional water-use data were collected for some States as part of a broader water-use data-collection program.

For 2000, self-supplied water withdrawals for the core categories of public supply, domestic, irrigation, industrial, and thermoelectric power were compiled for all States. Selfsupplied water withdrawals for livestock, aquaculture, and mining were compiled for selected States that represented most of the total water withdrawals for these categories during 1995. Data not reported for 2000 included self-supplied commercial withdrawals, deliveries from public supply for domestic, commercial, industrial, and thermoelectric-power purposes, and instream use for hydroelectric power. Also not reported were consumptive use, irrigation conveyance loss, reclaimed wastewater, number of wastewater facilities, and wastewater returns. The amount of power generated by thermoelectricor hydroelectric-power plants was not reported for 2000.

Although some States did not compile data for some categories for 2000, most of the water withdrawals are likely to be accounted for in the water-use estimates in this report. During 1995, 97 percent of total water withdrawals were used for public supply, domestic, irrigation, industrial, and thermoelectric power. All States collected and reported data for these categories for 2000 . The remaining 3 percent was used for livestock, animal specialties, mining, and commercial purposes. States that accounted for the majority of the water withdrawals for livestock, aquaculture, and mining uses for 1995 collected and reported data for 2000 .

Other changes for 2000 involved the reclassification of data within the thermoelectric-power and livestock categories. Thermoelectric-power water use was subdivided by cooling type (once-through or closed-loop) rather than by fuel type as in previous reports. Cooling-system type is the primary determinant of consumptive use relative to withdrawals at power plants. Most of the water withdrawn for once-through cooling is returned to the source and, therefore, generally results in a relatively smaller consumptive use. Water withdrawn for closedloop cooling is recirculated with a large part lost to evaporation, resulting in a larger consumptive use relative to withdrawals.

For 2000, the livestock category was no longer subdivided into livestock and animal specialties. Withdrawals for some animals that formerly were reported in the animal-specialties subcategory were included in the livestock category. The aquaculture category, new for 2000 , combined withdrawals for fish farms, formerly reported in the animal-specialties category, and fish hatcheries, formerly reported as self-supplied withdrawals in the commercial category.

\section{Sources of Data and Methods of Analysis}

The USGS, in cooperation with State and local agencies, compiled water-use estimates for 2000 for each county in the United States, the District of Columbia, Puerto Rico, and the U.S. Virgin Islands. The USGS National Water Use Information Program-implemented in 1978 to provide uniform, current, and reliable information on water use-coordinated the compilation effort. Water-use estimates and ancillary data were entered into a State aggregate water-use database in each USGS State office, and reviewed within the USGS and cooperating agencies. All water-use data compiled for this report are stored in the USGS Aggregate Water-Use Data System. Data collected for this report are available by county and State on the Internet at http://water.usgs.gov/watuse/.

Sources of information vary and are discussed for each category in subsequent parts of this report. Guidelines for preparing USGS water-use estimates for 2000 were developed and distributed to water-use study chiefs in each State office, and are available on the Internet site identified above. The following national data were available to each State: U.S. Environmental Protection Agency (USEPA) Safe Drinking Water Information System (SDWIS); U.S. Census Bureau, population; U.S. Department of Agriculture (USDA) Farm and Ranch Irrigation Survey; USDA Census of Agriculture; USDA, national agricultural statistics; and U.S. Department of Energy-Energy Information Administration (USDOEEIA), steam-electric plant statistics. Each USGS study chief was responsible for determining the most reliable sources of information available for estimating water use for their State.

Each USGS study chief compiled and analyzed information from various sources, made estimates of missing data, and prepared documentation that identified the sources of water-use information and methods used to determine water use for their State. Many States published reports on water use as part of the National Water Use Information Program. A list of these publications is available on the Internet at http://water.usgs.gov/watuse/.

\section{Acknowledgments}

The authors gratefully acknowledge the USGS study chiefs in each State who compiled the data for this report and the assistance provided by the many State and local agencies that shared data and expertise with the USGS. In many States, personnel from cooperating agencies worked as full partners with the USGS in this compilation effort. Cooperators include State agencies that manage water resources, operate data-collection programs, and administer regulations on water use and natural resources. The USGS water-use points of contact for each State are identified on the Internet at http://water.usgs.gov/watuse/. 


\section{TOtAL WATER Use}

Total water use in the United States for 2000 was determined from estimates of water withdrawals for the eight categories of public supply, domestic, irrigation, livestock, aquaculture, industrial, mining, and thermoelectric power (fig 1). Total freshwater and saline-water withdrawals for 2000 were estimated to be $408,000 \mathrm{Mgal} / \mathrm{d}$, or 457,000 thousand acre-feet per year (table 1). Freshwater withdrawals were 85 percent of the total, and the remaining 15 percent was saline water. Estimates of withdrawals by source indicate that for 2000, total surface-water withdrawals were $323,000 \mathrm{Mgal} / \mathrm{d}$, or 79 percent of the total withdrawals for all categories of use. About 81 percent of surface water withdrawn was freshwater. Total ground-water withdrawals were $84,500 \mathrm{Mgal} / \mathrm{d}$, of which 99 percent was freshwater. Nearly all (98 percent) saline-water withdrawals were from surface water.

Total withdrawals by category and State are listed in table 2. For 2000, the largest water withdrawals were for thermoelectric power and irrigation. Most water (195,000 Mgal/d) was withdrawn for thermoelectric power, of which 30 percent (59,500 Mgal/d) was saline. Illinois used the largest amount of freshwater for thermoelectric power (8 percent of the freshwater withdrawals for thermoelectric power). The largest saline withdrawals for thermoelectric power (41 percent) were in California and Florida. The largest freshwater withdrawals were for irrigation (40 percent of the total freshwater, or 137,000 Mgal/d). California used the largest amount of irrigation water and accounted for nearly one-quarter of the total irrigation withdrawals.

For 2000, more surface water than ground water was used in all categories except domestic, livestock, and mining (considering saline water only) (table 3). About 52 percent of the fresh surface-water withdrawals were for thermoelectric power and 30 percent were for irrigation. The largest fresh and saline surface-water withdrawals were in California. California accounted for the largest fresh surface-water withdrawals for public-supply, self-supplied domestic, irrigation, and livestock uses and for the largest saline surface-water withdrawals for thermoelectric-power use.
For 2000, most of the fresh ground-water withdrawals, 68 percent, were for irrigation (table 4). About 52 percent of the fresh ground-water use nationwide was in California, Texas, Nebraska, Arkansas, and Florida. About three-fourths of ground-water withdrawals in California and Texas were for irrigation; in Nebraska and Arkansas, 94 percent of the ground-water withdrawals were for irrigation. Ground-water withdrawals for public supply and irrigation in Florida were nearly identical. Nationwide, ground-water withdrawals for irrigation were about 3.5 times larger than the withdrawals for public supply. Only 1 percent of ground-water withdrawals were saline. Nearly all of the saline ground-water withdrawals were for mining, of which 40 percent were in Texas.

Figure 1 shows that the largest category of water withdrawals for 2000 was thermoelectric power (48 percent of total withdrawals). Irrigation accounted for 34 percent of the total withdrawals; public supply, 11 percent of the total; selfsupplied industrial, 5 percent of the total; and self-supplied domestic, livestock, aquaculture, and mining combined accounted for around 2 percent of the total withdrawals.

The geographic distribution of total, total surface-water, and total ground-water withdrawals is shown in figure 2. California, Texas, and Florida accounted for 25 percent of total withdrawals. California and Texas accounted for 17 percent of total surface-water withdrawals, and California accounted for 18 percent of total groundwater withdrawals. The geographic distribution of freshwater and saline-water withdrawals by State is shown in figure 3. California and Texas accounted for 18 percent of the total freshwater withdrawals, and California and Florida accounted for 40 percent of the total saline-water withdrawals.

A comparison of the intensity of freshwater withdrawals by State, in gallons per day per square mile, shows that some smaller- and medium-sized States in the eastern United States have a greater intensity of freshwater withdrawals per square mile compared to some larger-sized western States (fig. 4). California and Idaho also are high in water-use intensity; however, Texas, the State with the second largest total withdrawals, is relatively low in water-use intensity. 
Public Supply, 11 percent

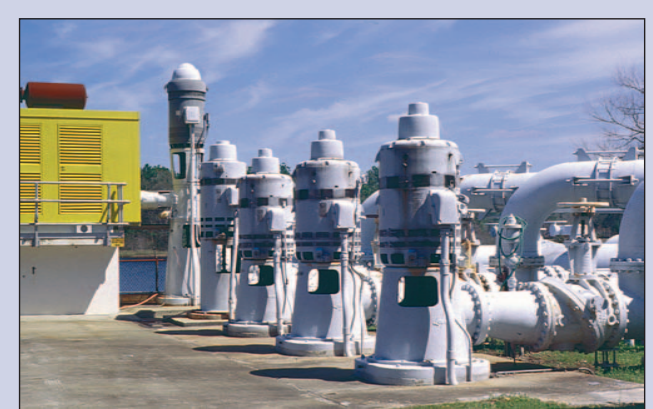

Public supply water intake, Bay County, Florida

Irrigation, 34 percent

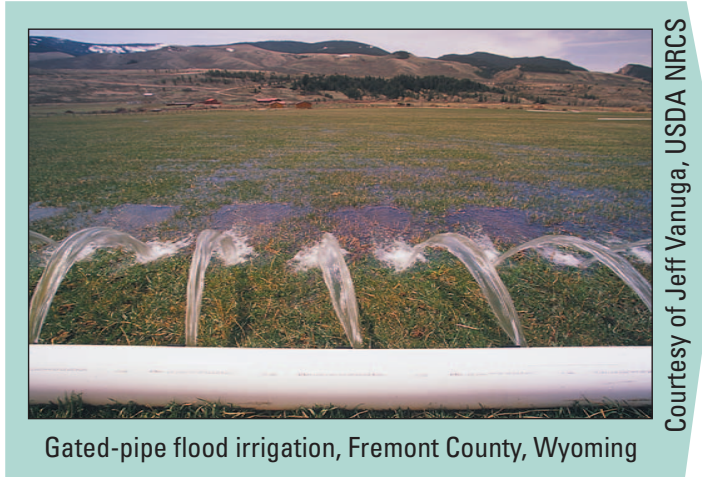

Aquaculture, less than 1 percent

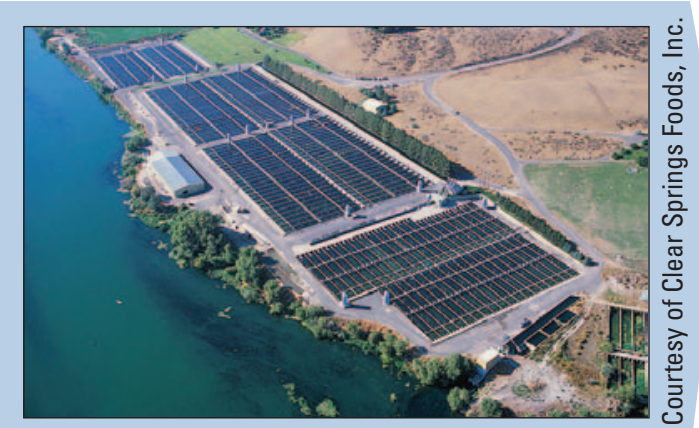

World's largest trout farm, Buhl, Idaho

Mining, less than 1 percent

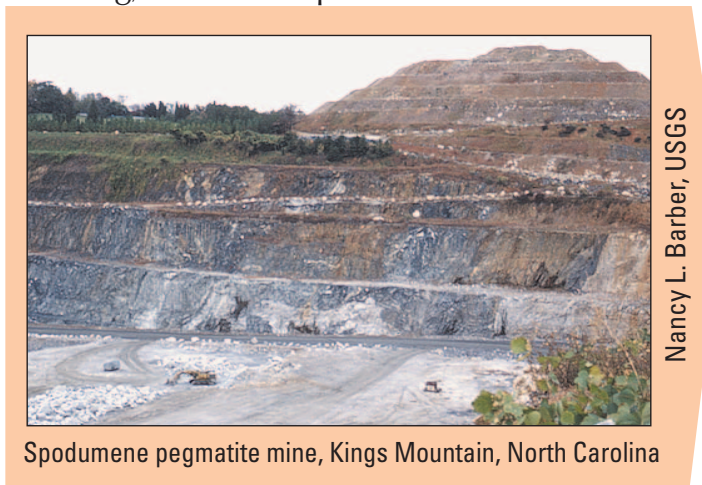

Domestic, less than 1 percent

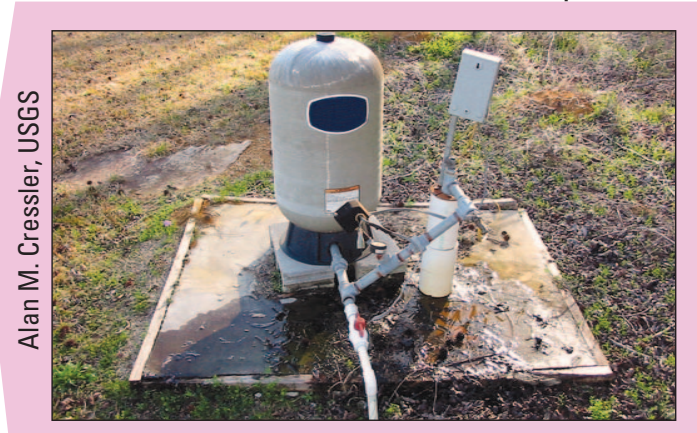

Domestic well, Early County, Georgia

Livestock, less than 1 percent

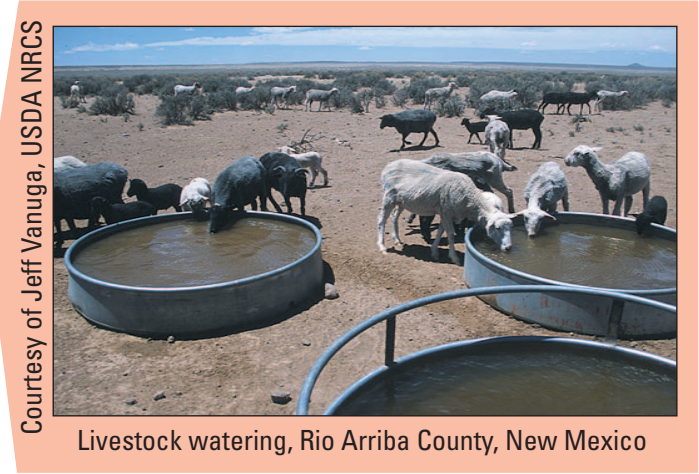

Industrial, 5 percent

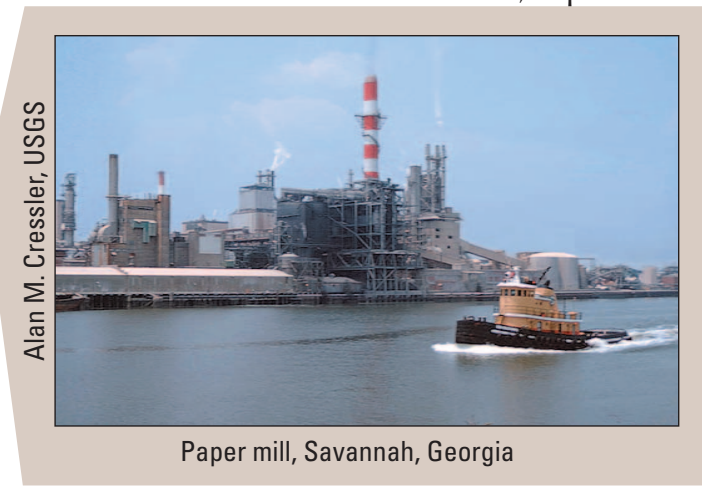

Thermoelectric Power, 48 percent

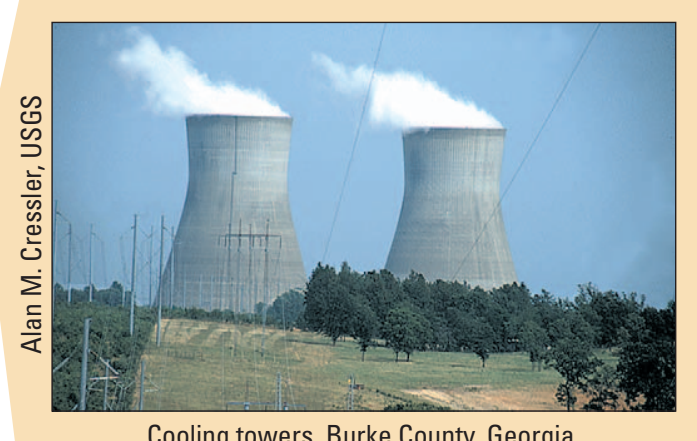

Figure 1. Total water withdrawals by category, 2000. 
Table 1. Total water withdrawals by source and State, 2000.

[Figures may not sum to totals because of independent rounding]

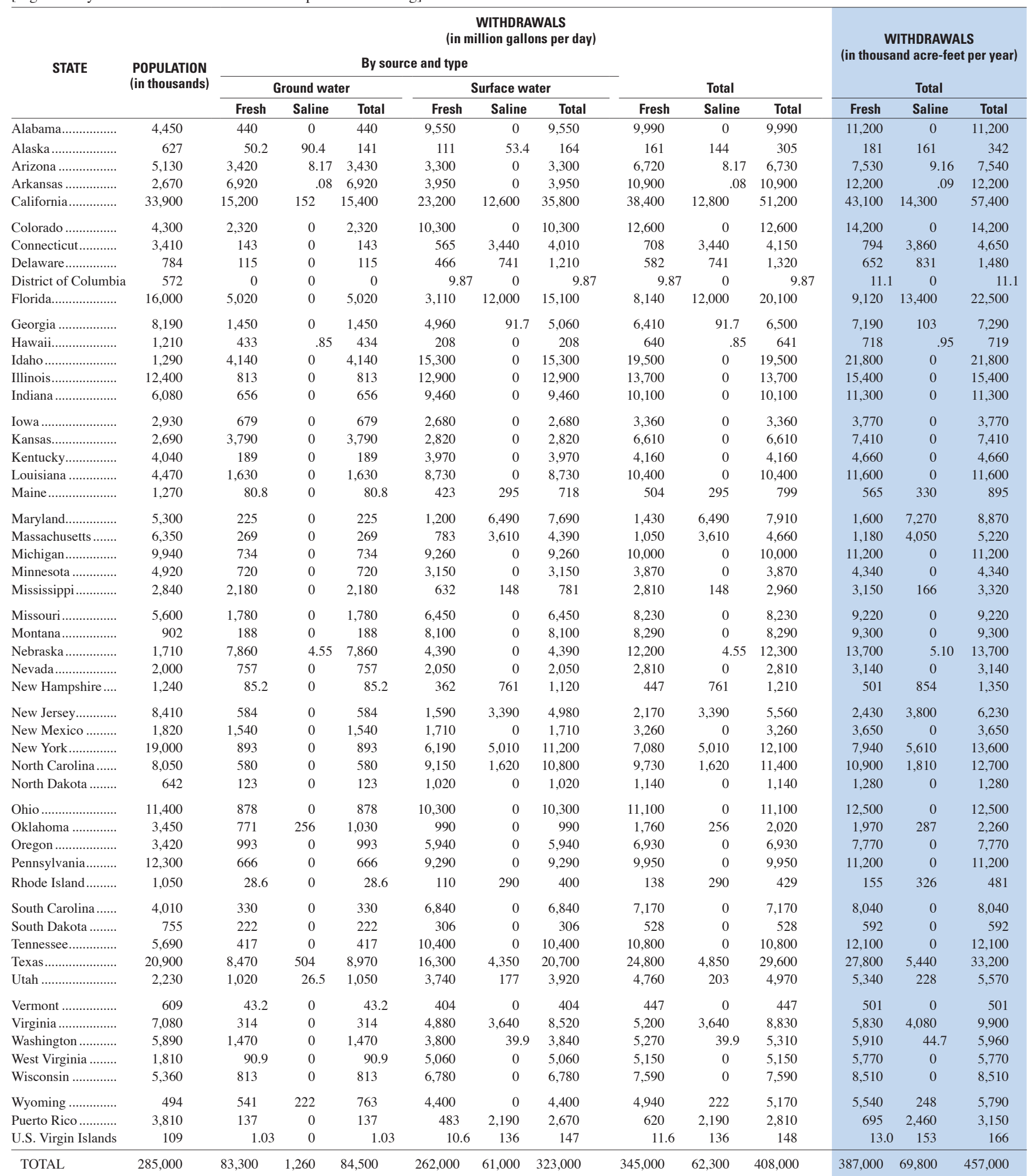


Table 2. Total water withdrawals by water-use category, 2000.

[Figures may not sum to totals because of independent rounding. All values are in million gallons per day. - , data not collected]

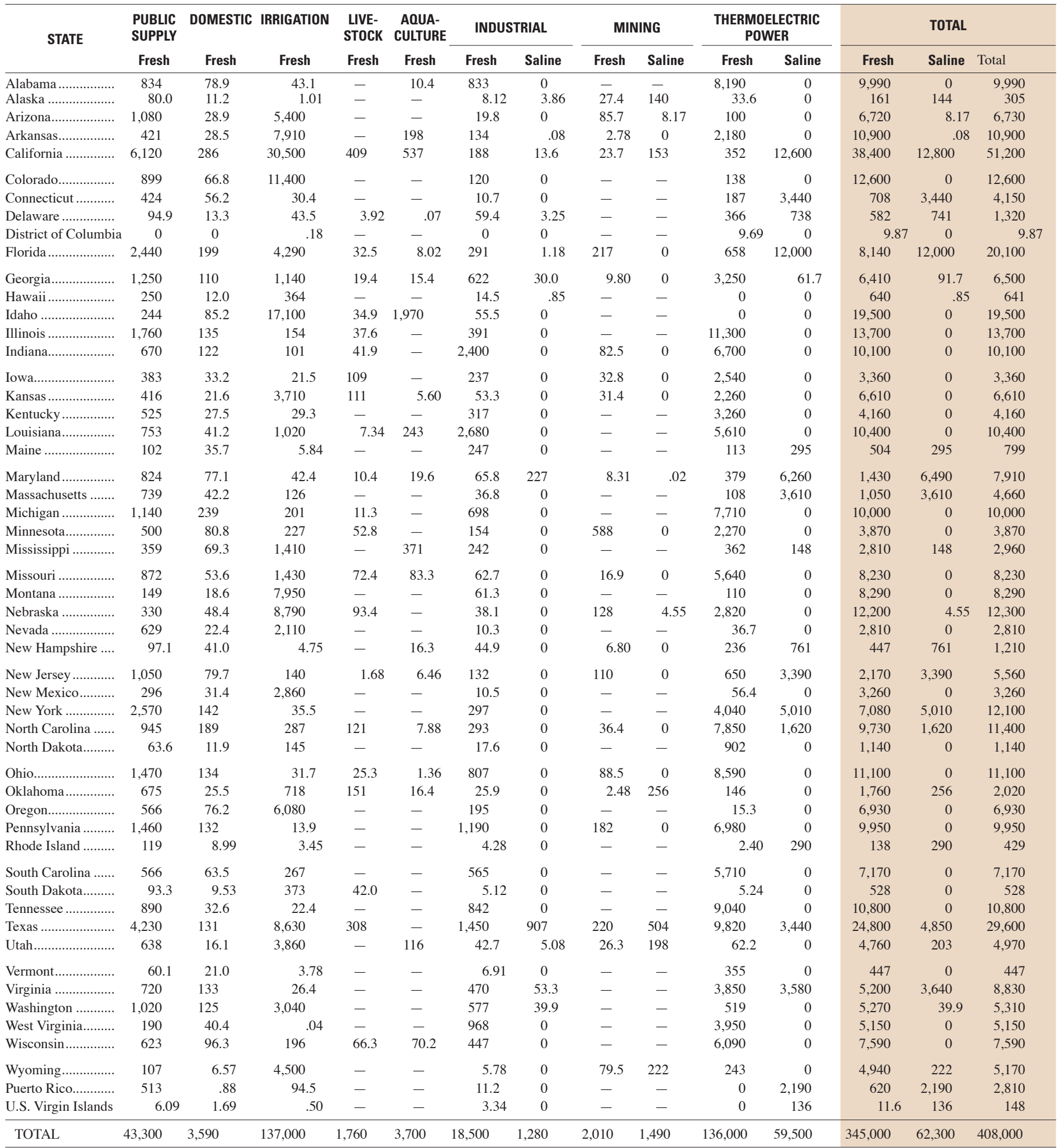


Table 3. Surface-water withdrawals by water-use category, 2000.

[Figures may not sum to totals because of independent rounding. All values are in million gallons per day. -, data not collected]

\begin{tabular}{|c|c|c|c|c|c|c|c|c|c|c|c|c|c|c|}
\hline \multirow{2}{*}{ STATE } & \multirow{2}{*}{$\begin{array}{c}\text { PUBLIC } \\
\text { SUPPLY } \\
\text { Fresh }\end{array}$} & \multirow{2}{*}{$\begin{array}{c}\text { DOMESTIC } \\
\text { Fresh }\end{array}$} & \multirow{2}{*}{$\begin{array}{l}\text { IRRIGATION } \\
\text { Fresh }\end{array}$} & \multirow{2}{*}{$\begin{array}{l}\text { LIVE- } \\
\text { STOCK } \\
\text { Fresh }\end{array}$} & \multirow{2}{*}{$\begin{array}{c}\text { AQUA- } \\
\text { CULTURE } \\
\text { Fresh }\end{array}$} & \multicolumn{2}{|c|}{ INDUSTRIAL } & \multicolumn{2}{|c|}{ MINING } & \multicolumn{2}{|c|}{$\begin{array}{c}\text { THERMOELECTRIC } \\
\text { POWER } \\
\end{array}$} & \multicolumn{3}{|c|}{ TOTAL } \\
\hline & & & & & & Fresh & Saline & Fresh & Saline & Fresh & Saline & Fresh & Saline & Total \\
\hline Alabama ........................ & 553 & 0 & 28.7 & - & 1.44 & 777 & 0 & - & - & 8,190 & 0 & 9,550 & 0 & 9,550 \\
\hline Alaska........................... & 50.7 & .25 & .02 & - & - & 3.80 & 3.86 & 27.4 & 49.5 & 28.9 & 0 & 111 & 53.4 & 164 \\
\hline Arizona ........................ & 613 & 0 & 2,660 & - & - & 0 & 0 & 4.43 & 0 & 26.2 & 0 & 3,300 & 0 & 3,300 \\
\hline Arkansas ...................... & 289 & 0 & 1,410 & - & 10.4 & 66.8 & 0 & 2.57 & 0 & 2,170 & 0 & 3,950 & 0 & 3,950 \\
\hline California...................... & 3,320 & 28.6 & 18,900 & 227 & 380 & 5.65 & 13.6 & 2.71 & .46 & 349 & 12,600 & 23,200 & 12,600 & 35,800 \\
\hline Colorado .......................... & 846 & 0 & 9,260 & - & - & 96.4 & 0 & - & - & 122 & 0 & 10,300 & 0 & 10,300 \\
\hline Connecticut.................. & 358 & 0 & 13.4 & - & - & 6.61 & 0 & - & - & 186 & 3,440 & 565 & 3,440 & 4,010 \\
\hline Delaware...................... & 49.8 & 0 & 7.89 & .22 & 0 & 42.5 & 3.25 & - & - & 366 & 738 & 466 & 741 & 1,210 \\
\hline District of Columbia & 0 & 0 & .18 & - & - & 0 & 0 & - & - & 9.69 & 0 & 9.87 & 0 & 9.87 \\
\hline 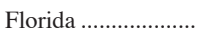 & 237 & 0 & 2,110 & 1.51 & .21 & 74.7 & 1.18 & 57.8 & 0 & 629 & 12,000 & 3,110 & 12,000 & 15,100 \\
\hline 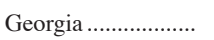 & 968 & 0 & 392 & 17.7 & 7.72 & 333 & 30.0 & 2.05 & 0 & 3,240 & 61.7 & 4,960 & 91.7 & 5,060 \\
\hline Hawaii ......................... & 7.60 & 7.22 & 193 & - & - & 0 & 0 & - & - & 0 & 0 & 208 & 0 & 208 \\
\hline Idaho ....................... & 25.3 & 0 & 13,300 & 7.20 & 1,920 & 19.7 & 0 & - & - & 0 & 0 & 15,300 & 0 & 15,300 \\
\hline Illinois.......................... & 1,410 & 0 & 4.25 & 0 & - & 259 & 0 & - & - & 11,300 & 0 & 12,900 & 0 & 12,900 \\
\hline 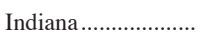 & 326 & 0 & 45.4 & 14.6 & - & 2,300 & 0 & 78.3 & 0 & 6,700 & 0 & 9,460 & 0 & 9,460 \\
\hline 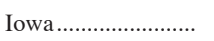 & 79.8 & 0 & 1.08 & 27.1 & - & 11.7 & 0 & 30.3 & 0 & 2,530 & 0 & 2,680 & 0 & 2,680 \\
\hline Kansas ........................ & 244 & 0 & 288 & 23.5 & 2.27 & 6.74 & 0 & 17.4 & 0 & 2,240 & 0 & 2,820 & 0 & 2,820 \\
\hline Kentucky .................... & 455 & 8.00 & 28.2 & - & - & 222 & 0 & - & - & 3,250 & 0 & 3,970 & 0 & 3,970 \\
\hline Louisiana ...................... & 404 & 0 & 232 & 3.31 & 115 & 2,400 & 0 & - & - & 5,580 & 0 & 8,730 & 0 & 8,730 \\
\hline 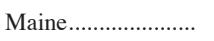 & 72.5 & 0 & 5.23 & - & - & 237 & 0 & - & - & 108 & 295 & 423 & 295 & 718 \\
\hline 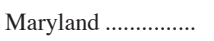 & 740 & 0 & 12.6 & 3.18 & 14.8 & 49.9 & 227 & 4.10 & .02 & 377 & 6,260 & 1,200 & 6,490 & 7,690 \\
\hline Massachusetts......... & 542 & 0 & 106 & - & - & 26.2 & 0 & - & - & 108 & 3,610 & 783 & 3,610 & 4,390 \\
\hline Michigan....................... & 896 & 0 & 73.2 & 1.15 & - & 589 & 0 & - & - & 7,710 & 0 & 9,260 & 0 & 9,260 \\
\hline Minnesota ...................... & 171 & 0 & 36.6 & 0 & - & 97.8 & 0 & 581 & 0 & 2,260 & 0 & 3,150 & 0 & 3,150 \\
\hline Mississippi................ & 40.4 & 0 & 99.1 & - & 49.8 & 124 & 0 & - & - & 318 & 148 & 632 & 148 & 781 \\
\hline Missouri.................... & 594 & 0 & 48.1 & 54.1 & 81.3 & 33.5 & 0 & 12.8 & 0 & 5,620 & 0 & 6,450 & 0 & 6,450 \\
\hline Montana ........................ & 92.4 & 1.29 & 7,870 & - & - & 29.3 & 0 & - & - & 110 & 0 & 8,100 & 0 & 8,100 \\
\hline Nebraska................................ & 63.8 & 0 & 1,370 & 17.4 & - & 2.60 & 0 & 122 & 0 & 2,810 & 0 & 4,390 & 0 & 4,390 \\
\hline Nevada......................... & 478 & 0 & 1,540 & - & - & 5.00 & 0 & - & - & 24.7 & 0 & 2,050 & 0 & 2,050 \\
\hline New Hampshire...... & 64.1 & .16 & 4.25 & - & 13.1 & 37.9 & 0 & 6.72 & 0 & 235 & 761 & 362 & 761 & 1,120 \\
\hline New Jersey .............. & 650 & 0 & 117 & 0 & 0 & 66.2 & 0 & 104 & 0 & 648 & 3,390 & 1,590 & 3,390 & 4,980 \\
\hline New Mexico ................ & 33.8 & 0 & 1,630 & - & - & 1.67 & 0 & - & - & 45.0 & 0 & 1,710 & 0 & 1,710 \\
\hline New York.................. & 1,980 & 0 & 12.1 & - & - & 152 & 0 & - & - & 4,040 & 5,010 & 6,190 & 5,010 & 11,200 \\
\hline North Carolina........ & 779 & 0 & 221 & 32.3 & 0 & 267 & 0 & 0 & 0 & 7,850 & 1,620 & 9,150 & 1,620 & 10,800 \\
\hline North Dakota ............ & 31.2 & 0 & 73.2 & - & - & 10.7 & 0 & - & - & 902 & 0 & 1,020 & 0 & 1,020 \\
\hline Ohio ............................. & 966 & 2.71 & 17.8 & 17.1 & 0 & 645 & 0 & 35.5 & 0 & 8,590 & 0 & 10,300 & 0 & 10,300 \\
\hline Oklahoma ................... & 562 & 0 & 151 & 97.2 & 16.1 & 19.1 & 0 & .23 & 0 & 143 & 0 & 990 & 0 & 990 \\
\hline Oregon ............................ & 447 & 7.97 & 5,290 & - & - & 183 & 0 & - & - & 12.8 & 0 & 5,940 & 0 & 5,940 \\
\hline Pennsylvania............ & 1,250 & 0 & 12.5 & - & - & 1,030 & 0 & 20.9 & 0 & 6,970 & 0 & 9,290 & 0 & 9,290 \\
\hline Rhode Island............. & 102 & 0 & 2.99 & - & - & 2.09 & 0 & - & - & 2.40 & 290 & 110 & 290 & 400 \\
\hline South Carolina........ & 462 & 0 & 162 & - & - & 514 & 0 & - & - & 5,700 & 0 & 6,840 & 0 & 6,840 \\
\hline South Dakota ........... & 39.1 & .01 & 236 & 25.2 & - & 1.96 & 0 & - & - & 4.01 & 0 & 306 & 0 & 306 \\
\hline Tennessee................... & 569 & 0 & 15.1 & - & - & 785 & 0 & - & - & 9,040 & 0 & 10,400 & 0 & 10,400 \\
\hline Texas.................................. & 2,970 & 0 & 2,130 & 172 & - & 1,200 & 906 & 91.5 & 0 & 9,760 & 3,440 & 16,300 & 4,350 & 20,700 \\
\hline Utah ............................. & 274 & 0 & 3,390 & - & 0 & 8.38 & 0 & 17.7 & 177 & 49.2 & 0 & 3,740 & 177 & 3,920 \\
\hline Vermont ........................ & 40.6 & .25 & 3.45 & - & - & 4.86 & 0 & - & - & 355 & 0 & 404 & 0 & 404 \\
\hline Virginia ........................ & 650 & 0 & 22.8 & - & - & 365 & 53.3 & - & - & 3,850 & 3,580 & 4,880 & 3,640 & 8,520 \\
\hline Washington ............... & 552 & .02 & 2,290 & - & - & 439 & 39.9 & - & - & 518 & 0 & 3,800 & 39.9 & 3,840 \\
\hline West Virginia ........... & 149 & .81 & .02 & - & - & 958 & 0 & - & - & 3,950 & 0 & 5,060 & 0 & 5,060 \\
\hline 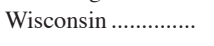 & 293 & 0 & 1.57 & 6.02 & 30.4 & 364 & 0 & - & - & 6,090 & 0 & 6,780 & 0 & 6,780 \\
\hline 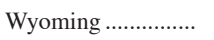 & 49.4 & 0 & 4,090 & - & - & 1.47 & 0 & 20.7 & 0 & 242 & 0 & 4,400 & 0 & 4,400 \\
\hline Puerto Rico.............. & 425 & 0 & 57.5 & - & - & 0 & 0 & - & - & 0 & 2,190 & 483 & 2,190 & 2,670 \\
\hline U.S. Virgin Islands & 5.57 & 1.69 & .21 & - & - & 3.12 & 0 & - & - & 0 & 136 & 10.6 & 136 & 147 \\
\hline TOTAL & 27,300 & 58.9 & 80,000 & 747 & 2,640 & 14,900 & 1,280 & 1,240 & 227 & 135,000 & 59,500 & 262,000 & 61,000 & 323,000 \\
\hline
\end{tabular}


Table 4. Ground-water withdrawals by water-use category, 2000.

[Figures may not sum to totals because of independent rounding. All values are in million gallons per day. - , data not collected]

\begin{tabular}{|c|c|c|c|c|c|c|c|c|c|c|c|c|c|}
\hline \multirow{2}{*}{ STATE } & \multirow{2}{*}{$\begin{array}{c}\text { PUBLIC } \\
\text { SUPPLY } \\
\text { Fresh }\end{array}$} & \multirow{2}{*}{$\begin{array}{c}\text { DOMESTIC } \\
\text { Fresh }\end{array}$} & \multirow{2}{*}{$\begin{array}{l}\text { IRRIGATION } \\
\text { Fresh }\end{array}$} & \multirow{2}{*}{$\begin{array}{l}\text { LIVE- } \\
\text { STOCK } \\
\text { Fresh }\end{array}$} & \multirow{2}{*}{$\begin{array}{l}\text { AQUA- } \\
\text { CULTURE } \\
\text { Fresh }\end{array}$} & \multicolumn{2}{|c|}{ INDUSTRIAL } & \multicolumn{2}{|c|}{ MINING } & \multirow{2}{*}{$\begin{array}{c}\text { THERMOELECTRIC } \\
\text { POWER }\end{array}$} & \multicolumn{3}{|c|}{ TOTAL } \\
\hline & & & & & & Fresh & Saline & Fresh & Saline & & Fresh & Saline & Total \\
\hline Alabama .................... & 281 & 78.9 & 14.5 & - & 8.93 & 56.0 & 0 & - & - & 0 & 440 & 0 & 440 \\
\hline Alaska ............................ & 29.3 & 10.9 & .99 & - & - & 4.32 & 0 & .01 & 90.4 & 4.65 & 50.2 & 90.4 & 141 \\
\hline 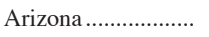 & 469 & 28.9 & 2,750 & - & - & 19.8 & 0 & 81.2 & 8.17 & 74.3 & 3,420 & 8.17 & 3,430 \\
\hline Arkansas ..................... & 132 & 28.5 & 6,510 & - & 187 & 67.0 & .08 & .21 & 0 & 2.92 & 6,920 & .08 & 6,920 \\
\hline California.................... & 2,800 & 257 & 11,600 & 182 & 158 & 183 & 0 & 21.0 & 152 & 3.23 & 15,200 & 152 & 15,400 \\
\hline 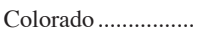 & 53.7 & 66.8 & 2,160 & - & - & 23.6 & 0 & - & - & 16.1 & 2,320 & 0 & 2,320 \\
\hline Connecticut.............. & 66.0 & 56.2 & 17.0 & - & - & 4.13 & 0 & - & - & .08 & 143 & 0 & 143 \\
\hline Delaware ..................... & 45.0 & 13.3 & 35.6 & 3.70 & .07 & 17.0 & 0 & - & - & .47 & 115 & 0 & 115 \\
\hline District of Columbia & 0 & 0 & 0 & - & - & 0 & 0 & - & - & 0 & 0 & 0 & 0 \\
\hline Florida .......................... & 2,200 & 199 & 2,180 & 31.0 & 7.81 & 216 & 0 & 160 & 0 & 29.5 & 5,020 & 0 & 5,020 \\
\hline 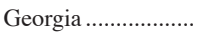 & 278 & 110 & 750 & 1.66 & 7.70 & 290 & 0 & 7.75 & 0 & 1.03 & 1,450 & 0 & 1,450 \\
\hline 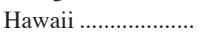 & 243 & 4.82 & 171 & - & - & 14.5 & .85 & - & - & 0 & 433 & .85 & 434 \\
\hline Idaho ............................. & 219 & 85.2 & 3,720 & 27.7 & 51.5 & 35.8 & 0 & - & - & 0 & 4,140 & 0 & 4,140 \\
\hline 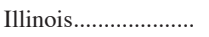 & 353 & 135 & 150 & 37.6 & - & 132 & 0 & - & - & 5.75 & 813 & 0 & 813 \\
\hline 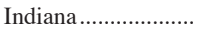 & 345 & 122 & 55.5 & 27.3 & - & 99.7 & 0 & 4.20 & 0 & 2.58 & 656 & 0 & 656 \\
\hline 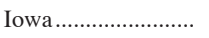 & 303 & 33.2 & 20.4 & 81.8 & - & 226 & 0 & 2.49 & 0 & 11.9 & 679 & 0 & 679 \\
\hline 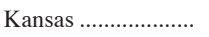 & 172 & 21.6 & 3,430 & 87.2 & 3.33 & 46.6 & 0 & 14.0 & 0 & 14.9 & 3,790 & 0 & 3,790 \\
\hline Kentucky ................... & 71.0 & 19.5 & 1.14 & - & - & 95.2 & 0 & - & - & 2.71 & 189 & 0 & 189 \\
\hline Louisiana ..................... & 349 & 41.2 & 791 & 4.03 & 128 & 285 & 0 & - & - & 28.4 & 1,630 & 0 & 1,630 \\
\hline Maine............................. & 29.6 & 35.7 & .61 & - & - & 9.90 & 0 & - & - & 4.92 & 80.8 & 0 & 80.8 \\
\hline Maryland .................... & 84.6 & 77.1 & 29.8 & 7.18 & 4.81 & 15.9 & 0 & 4.21 & 0 & 1.80 & 225 & 0 & 225 \\
\hline Massachusetts......... & 197 & 42.2 & 19.7 & - & - & 10.7 & 0 & - & - & 0 & 269 & 0 & 269 \\
\hline Michigan.................... & 247 & 239 & 128 & 10.2 & - & 110 & 0 & - & - & 0 & 734 & 0 & 734 \\
\hline Minnesota .................. & 329 & 80.8 & 190 & 52.8 & - & 56.3 & 0 & 6.90 & 0 & 4.17 & 720 & 0 & 720 \\
\hline Mississippi..................... & 319 & 69.3 & 1,310 & - & 321 & 118 & 0 & - & - & 43.5 & 2,180 & 0 & 2,180 \\
\hline Missouri.................... & 278 & 53.6 & 1,380 & 18.3 & 2.01 & 29.2 & 0 & 4.10 & 0 & 12.2 & 1,780 & 0 & 1,780 \\
\hline Montana...................... & 56.1 & 17.3 & 83.0 & - & - & 31.9 & 0 & - & - & 0 & 188 & 0 & 188 \\
\hline Nebraska..................... & 266 & 48.4 & 7,420 & 76.0 & - & 35.5 & 0 & 5.64 & 4.55 & 6.87 & 7,860 & 4.55 & 7,860 \\
\hline 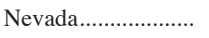 & 151 & 22.4 & 567 & - & - & 5.29 & 0 & - & - & 12.0 & 757 & 0 & 757 \\
\hline New Hampshire..... & 33.0 & 40.9 & .50 & - & 3.12 & 6.95 & 0 & .08 & 0 & .71 & 85.2 & 0 & 85.2 \\
\hline New Jersey ............. & 400 & 79.7 & 22.8 & 1.68 & 6.46 & 65.3 & 0 & 6.12 & 0 & 2.24 & 584 & 0 & 584 \\
\hline New Mexico ........... & 262 & 31.4 & 1,230 & - & - & 8.80 & 0 & - & - & 11.4 & 1,540 & 0 & 1,540 \\
\hline New York..................... & 583 & 142 & 23.3 & - & - & 145 & 0 & - & - & 0 & 893 & 0 & 893 \\
\hline North Carolina........ & 166 & 189 & 65.8 & 89.1 & 7.88 & 25.6 & 0 & 36.4 & 0 & .09 & 580 & 0 & 580 \\
\hline North Dakota .......... & 32.4 & 11.9 & 72.2 & - & - & 6.88 & 0 & - & - & 0 & 123 & 0 & 123 \\
\hline 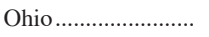 & 500 & 132 & 13.9 & 8.20 & 1.36 & 162 & 0 & 53.1 & 0 & 7.57 & 878 & 0 & 878 \\
\hline Oklahoma .................. & 113 & 25.5 & 566 & 53.6 & .29 & 6.83 & 0 & 2.25 & 256 & 3.27 & 771 & 256 & 1,030 \\
\hline Oregon ........................ & 118 & 68.3 & 792 & - & - & 12.1 & 0 & - & - & 2.47 & 993 & 0 & 993 \\
\hline Pennsylvania........... & 212 & 132 & 1.38 & - & - & 155 & 0 & 162 & 0 & 3.98 & 666 & 0 & 666 \\
\hline Rhode Island........... & 16.9 & 8.99 & .46 & - & - & 2.19 & 0 & - & - & 0 & 28.6 & 0 & 28.6 \\
\hline South Carolina........ & 105 & 63.5 & 106 & - & - & 50.9 & 0 & - & - & 5.83 & 330 & 0 & 330 \\
\hline South Dakota .......... & 54.2 & 9.52 & 137 & 16.9 & - & 3.16 & 0 & - & - & 1.23 & 222 & 0 & 222 \\
\hline Tennessee................... & 321 & 32.6 & 7.33 & - & - & 56.3 & 0 & - & - & 0 & 417 & 0 & 417 \\
\hline Texas............................ & 1,260 & 131 & 6,500 & 137 & - & 244 & .50 & 129 & 504 & 60.2 & 8,470 & 504 & 8,970 \\
\hline 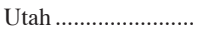 & 364 & 16.1 & 469 & - & 116 & 34.3 & 5.08 & 8.60 & 21.5 & 13.1 & 1,020 & 26.5 & 1,050 \\
\hline 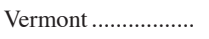 & 19.5 & 20.7 & .33 & - & - & 2.05 & 0 & - & - & .66 & 43.2 & 0 & 43.2 \\
\hline Virginia ...................... & 70.7 & 133 & 3.57 & - & - & 104 & 0 & - & - & 1.50 & 314 & 0 & 314 \\
\hline Washington ............. & 464 & 125 & 747 & - & - & 138 & 0 & - & - & .92 & 1,470 & 0 & 1,470 \\
\hline West Virginia ........... & 41.6 & 39.6 & .02 & - & - & 9.70 & 0 & - & - & 0 & 90.9 & 0 & 90.9 \\
\hline Wisconsin .................. & 330 & 96.3 & 195 & 60.3 & 39.8 & 83.0 & 0 & - & - & 8.99 & 813 & 0 & 813 \\
\hline 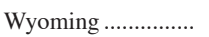 & 57.2 & 6.57 & 413 & - & - & 4.31 & 0 & 58.8 & 222 & 1.13 & 541 & 222 & 763 \\
\hline Puerto Rico ............. & 88.5 & .88 & 36.9 & - & - & 11.2 & 0 & - & - & 0 & 137 & 0 & 137 \\
\hline U.S. Virgin Islands & .52 & 0 & .29 & - & - & .22 & 0 & - & - & 0 & 1.03 & 0 & 1.03 \\
\hline TOTAL & 16,000 & 3,530 & 56,900 & 1,010 & 1,060 & 3,570 & 6.51 & 767 & 1,260 & 409 & 83,300 & 1,260 & 84,500 \\
\hline
\end{tabular}



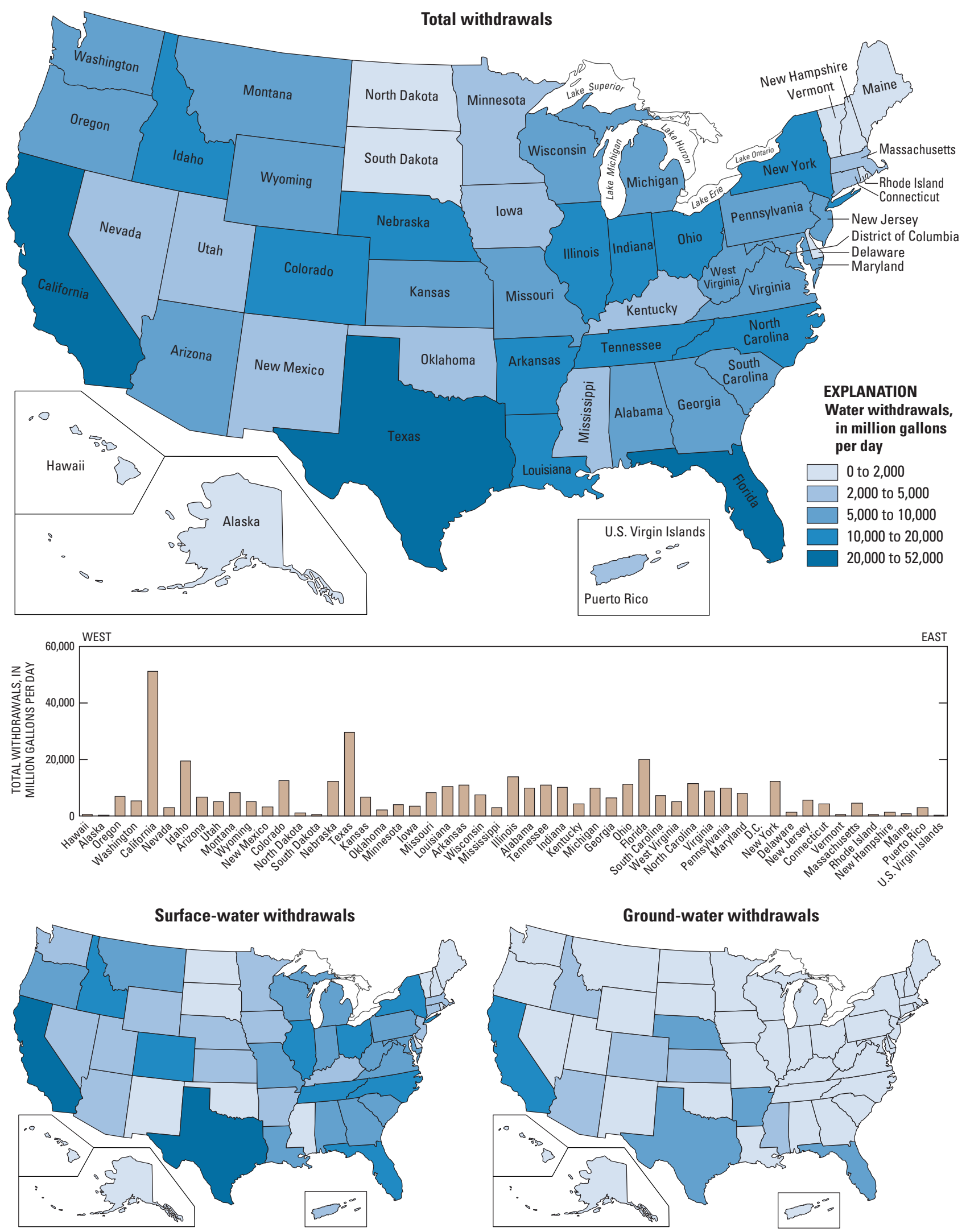

Figure 2. Total, surface-water, and ground-water withdrawals, 2000. 

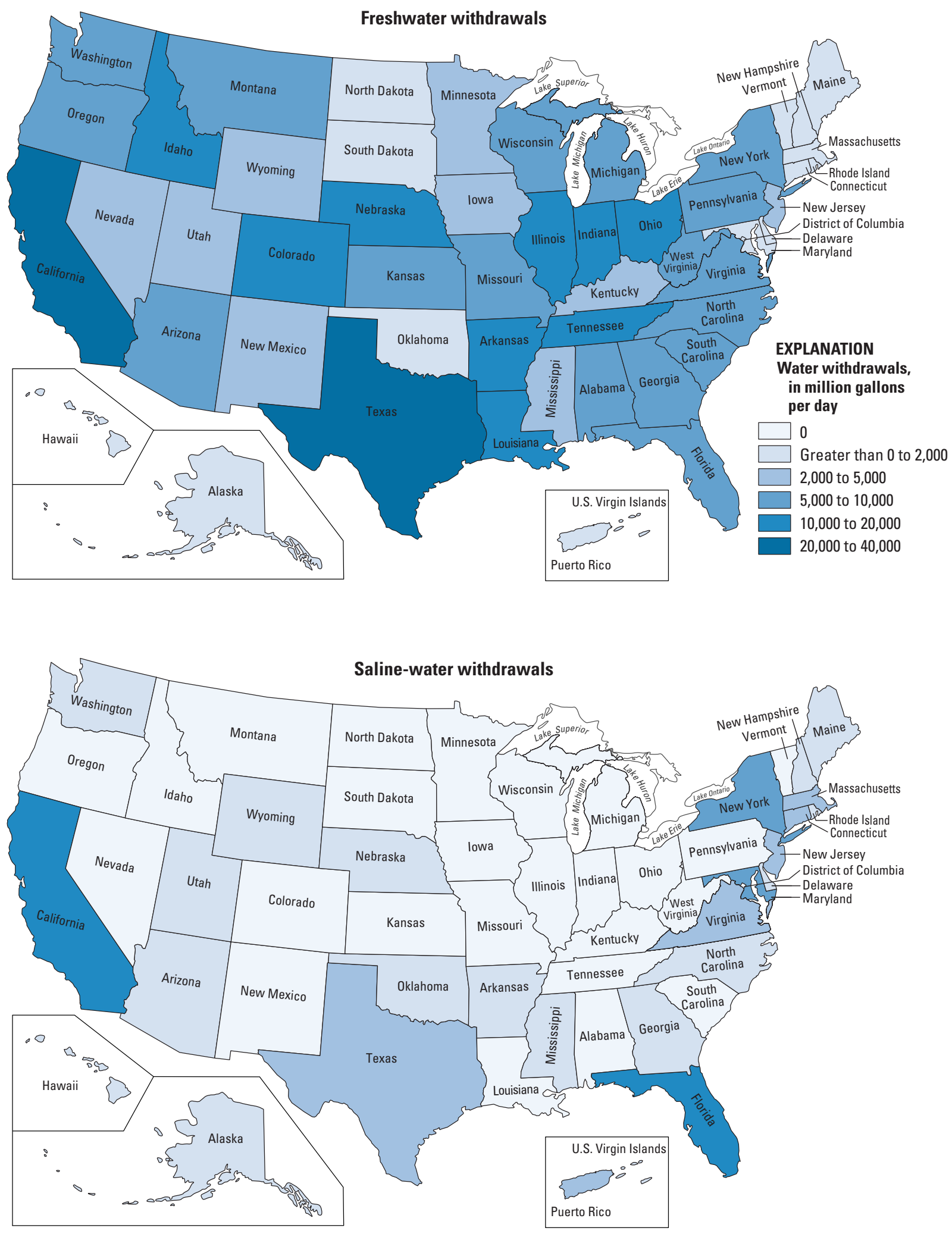

Figure 3. Fresh and saline water withdrawals, 2000. 


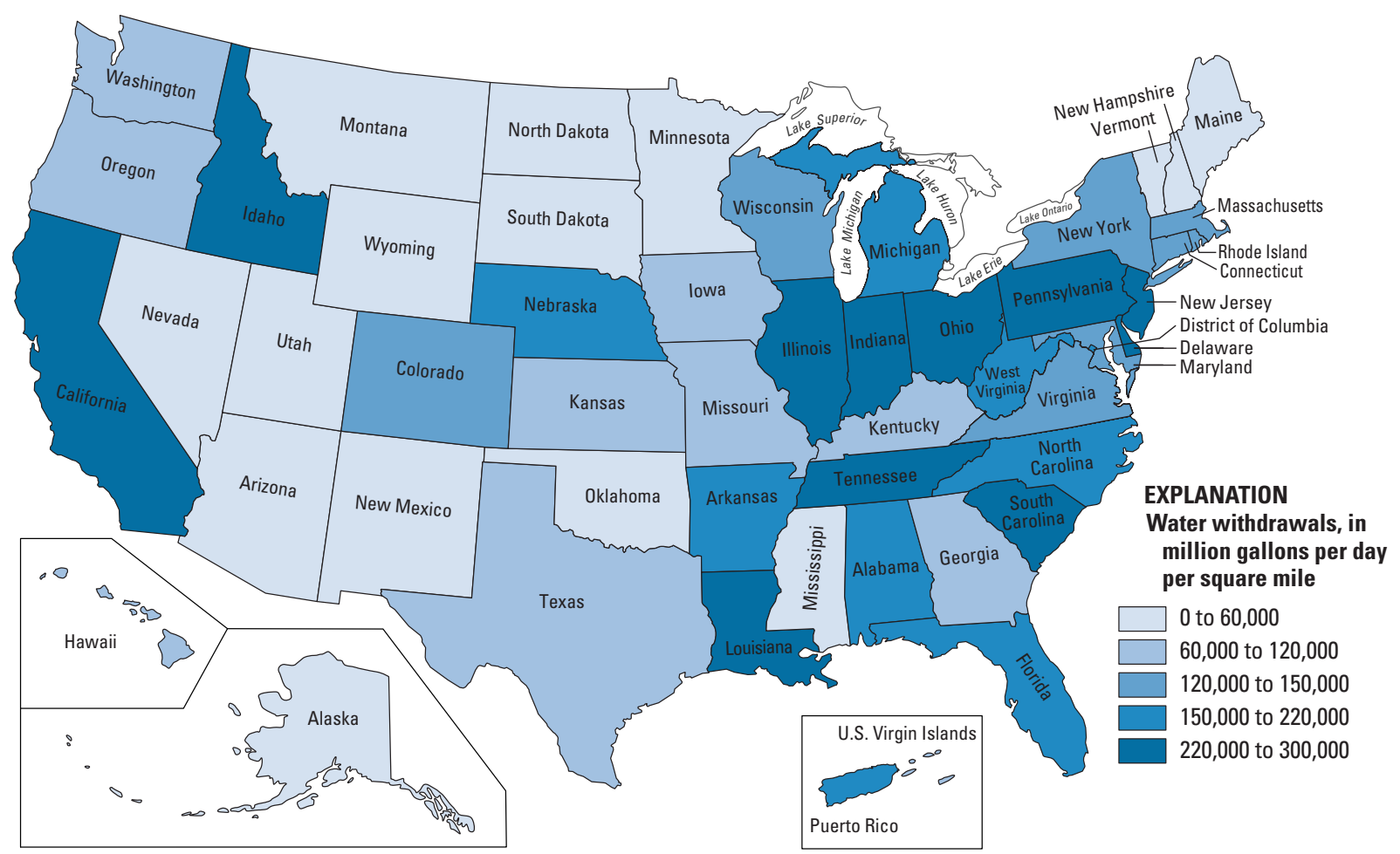

Figure 4. Intensity of freshwater withdrawals, 2000.

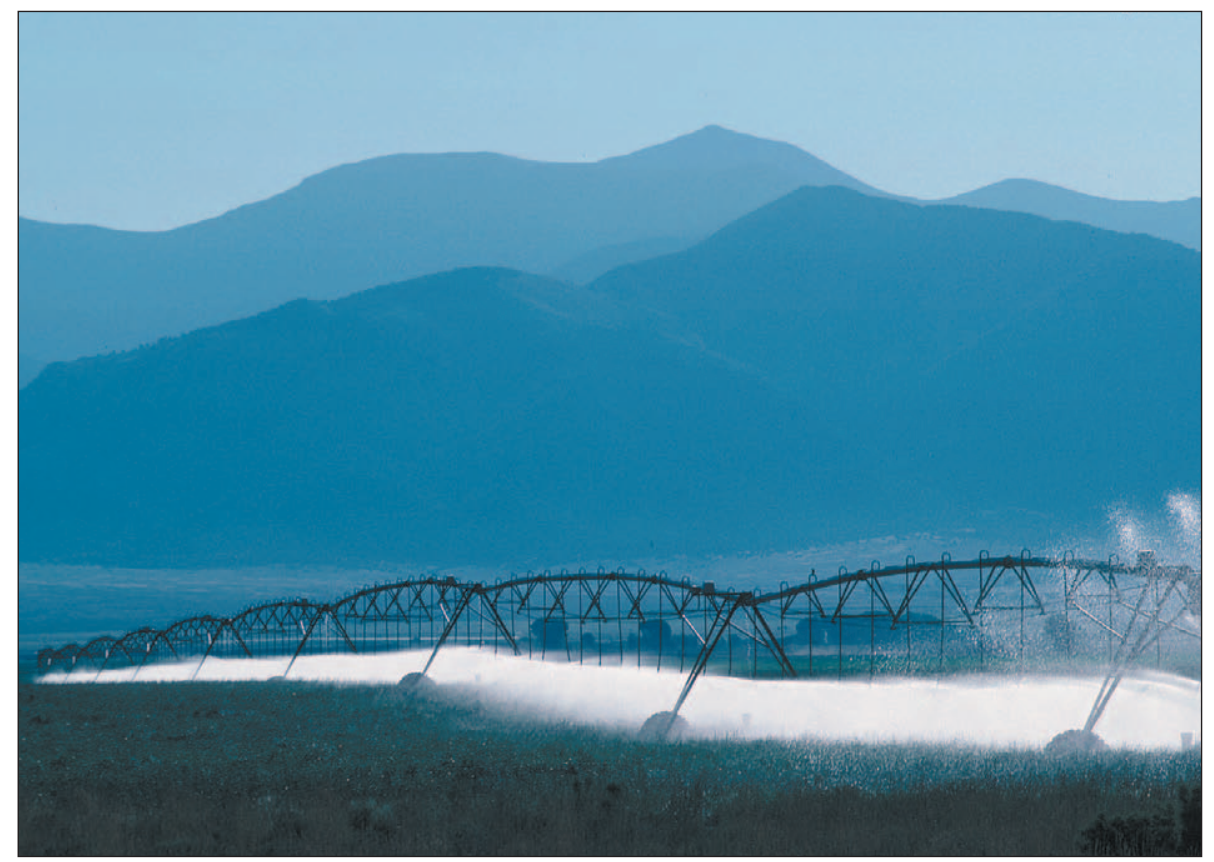

A sprinkler irrigation system in Colorado. Efficient irrigation systems help save water and decrease leaching of salts. (Photo courtesy of Gene Alexander, USDA Natural Resources Conservation Services.) 
Public supply refers to water withdrawn by public and private water suppliers that furnish water to at least 25 people or have a minimum of 15 connections. Publicsupply water may be delivered to users for domestic, commercial, industrial, or thermoelectric-power purposes. Some public-supply water may be delivered to other public suppliers or used in the processes of water and wastewater treatment. Public-supply water is used for such public services (public uses) as pools, parks, and public buildings; or be unaccounted for (losses) because of system leaks or such nonmetered services as firefighting or the flushing of water lines. Some public suppliers treat saline water before it is distributed. However, all public-supply withdrawals in this report are considered freshwater. Estimates for water deliveries, public use, and losses were not reported for 2000 for public supply.

Public-supply withdrawals and population are listed by State in table 5. For 2000, withdrawals were an estimated 43,300 Mgal/d, or 48,500 thousand acre-feet per year. Public-supply withdrawals were about 13 percent of total freshwater withdrawals and nearly 21 percent of total freshwater withdrawals for all categories excluding thermoelectric power. About 242 million people depended on water from public suppliers. The majority of the water for public supply (63 percent) was withdrawn from surface sources. Between 1995 and 2000, public-supply withdrawals and the population served increased 8 percent. The population served as a percentage of the total U.S. population was about 85 percent in 2000 .

The geographic distribution of total, surface-water, and ground-water withdrawals for public supply is shown in figure 5. States with the largest populations withdrew the largest quantities of water. California, Texas, New York, Florida, and Illinois accounted for 40 percent of total public-supply withdrawals and 38 percent of the total population served by public suppliers. The largest surface-water withdrawals were in California and Texas, and the largest ground-water withdrawals were in California and Florida.

The source of data used for estimating public-supply water withdrawals by source of supply and population served varied by State. Public suppliers; State health, environmental, or water-permitting agencies; and the USEPA SDWIS database were the primary sources of information.

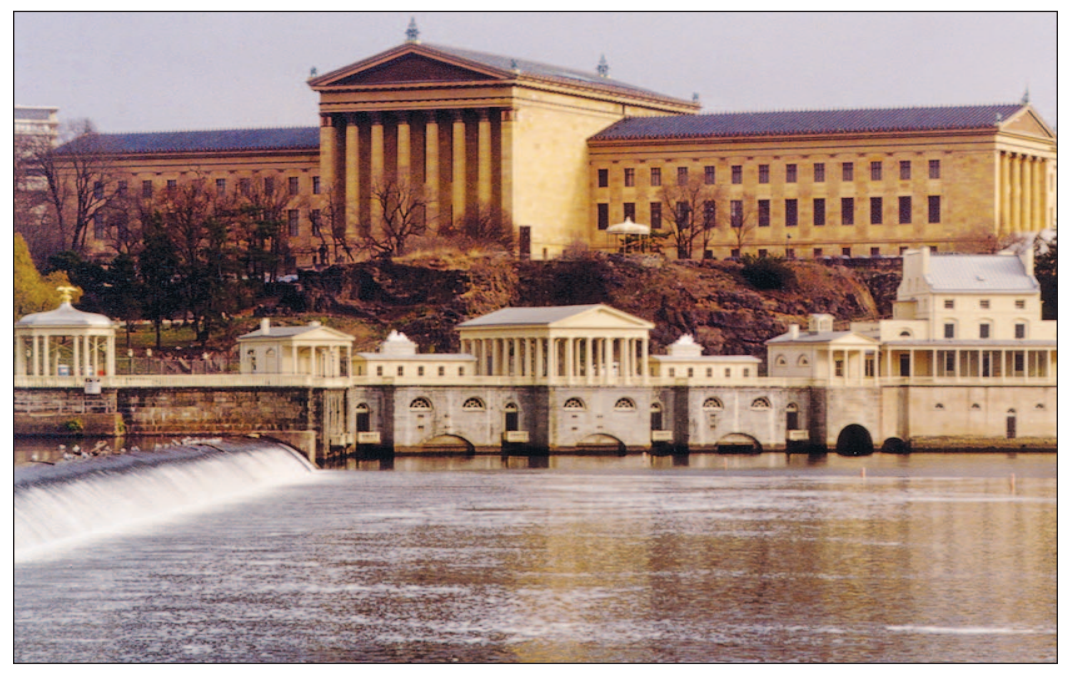

Fairmont Water Works (1815-1911), Philadelphia, Pa.

(Photo courtesy of Philadelphia Water Department.) 
Table 5. Public-supply water withdrawals, 2000.

[Figures may not sum to totals because of independent rounding]

\begin{tabular}{|c|c|c|c|c|c|c|c|c|c|}
\hline \multirow{3}{*}{ STATE } & \multicolumn{3}{|c|}{$\begin{array}{l}\text { POPULATION } \\
\text { (in thousands) }\end{array}$} & \multicolumn{3}{|c|}{$\begin{array}{c}\text { WITHDRAWALS } \\
\text { (in million gallons per day) }\end{array}$} & \multicolumn{3}{|c|}{$\begin{array}{c}\text { WITHDRAWALS } \\
\text { (in thousand acre-feet per year) }\end{array}$} \\
\hline & \multirow[b]{2}{*}{ Total } & \multicolumn{2}{|c|}{ Served by public supply } & \multicolumn{2}{|c|}{ By source } & \multirow[b]{2}{*}{ Total } & \multicolumn{2}{|r|}{ By source } & \multirow[b]{2}{*}{ Total } \\
\hline & & Population & $\begin{array}{l}\text { Population } \\
\text { (in percent) }\end{array}$ & $\begin{array}{l}\text { Ground } \\
\text { water }\end{array}$ & $\begin{array}{l}\text { Surface } \\
\text { water }\end{array}$ & & $\begin{array}{l}\text { Ground } \\
\text { water }\end{array}$ & $\begin{array}{c}\text { Surface } \\
\text { water }\end{array}$ & \\
\hline Alabama ………........ & 4,450 & 3,580 & 80 & 281 & 553 & 834 & 315 & 620 & 935 \\
\hline Alaska .......................... & 627 & 421 & 67 & 29.3 & 50.7 & 80.0 & 32.9 & 56.9 & 89.7 \\
\hline Arizona ..................... & 5,130 & 4,870 & 95 & 469 & 613 & 1,080 & 526 & 688 & 1,210 \\
\hline Arkansas .................... & 2,670 & 2,320 & 87 & 132 & 289 & 421 & 148 & 324 & 472 \\
\hline California ................... & 33,900 & 30,100 & 89 & 2,800 & 3,320 & 6,120 & 3,140 & 3,730 & 6,860 \\
\hline Colorado .................... & 4,300 & 3,750 & 87 & 53.7 & 846 & 899 & 60.2 & 948 & 1,010 \\
\hline Connecticut ............ & 3,410 & 2,660 & 78 & 66.0 & 358 & 424 & 74.0 & 402 & 476 \\
\hline Delaware ................. & 784 & 617 & 79 & 45.0 & 49.8 & 94.9 & 50.5 & 55.9 & 106 \\
\hline District of Columbia & 572 & 572 & 100 & 0 & 0 & 0 & 0 & 0 & 0 \\
\hline Florida ……............... & 16,000 & 14,000 & 88 & 2,200 & 237 & 2,440 & 2,470 & 266 & 2,730 \\
\hline Georgia ....................... & 8,190 & 6,730 & 82 & 278 & 968 & 1,250 & 311 & 1,090 & 1,400 \\
\hline 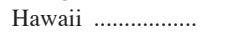 & 1,210 & 1,140 & 94 & 243 & 7.60 & 250 & 272 & 8.52 & 281 \\
\hline Idaho ............................ & 1,290 & 928 & 72 & 219 & 25.3 & 244 & 245 & 28.3 & 274 \\
\hline Illinois ............................ & 12,400 & 10,900 & 88 & 353 & 1,410 & 1,760 & 396 & 1,580 & 1,970 \\
\hline Indiana ……................... & 6,080 & 4,480 & 74 & 345 & 326 & 670 & 386 & 365 & 751 \\
\hline Iowa ………….......... & 2,930 & 2,410 & 83 & 303 & 79.8 & 383 & 340 & 89.5 & 429 \\
\hline Kansas ........................ & 2,690 & 2,500 & 93 & 172 & 244 & 416 & 193 & 273 & 466 \\
\hline Kentucky ................... & 4,040 & 3,490 & 86 & 71.0 & 455 & 525 & 79.5 & 510 & 589 \\
\hline Louisiana .................. & 4,470 & 3,950 & 88 & 349 & 404 & 753 & 392 & 453 & 844 \\
\hline Maine …………............ & 1,270 & 726 & 57 & 29.6 & 72.5 & 102 & 33.2 & 81.3 & 115 \\
\hline Maryland ................ & 5,300 & 4,360 & 82 & 84.6 & 740 & 824 & 94.8 & 829 & 924 \\
\hline Massachusetts ....... & 6,350 & 5,880 & 93 & 197 & 542 & 739 & 220 & 608 & 828 \\
\hline Michigan ..................... & 9,940 & 7,170 & 72 & 247 & 896 & 1,140 & 277 & 1,000 & 1,280 \\
\hline Minnesota ............... & 4,920 & 3,770 & 77 & 329 & 171 & 500 & 369 & 192 & 561 \\
\hline Mississippi ............. & 2,840 & 2,190 & 77 & 319 & 40.4 & 359 & 357 & 45.3 & 402 \\
\hline Missouri ……….......... & 5,600 & 4,770 & 85 & 278 & 594 & 872 & 311 & 666 & 978 \\
\hline Montana ...................... & 902 & 664 & 74 & 56.1 & 92.4 & 149 & 62.9 & 104 & 167 \\
\hline Nebraska ................... & 1,710 & 1,390 & 81 & 266 & 63.8 & 330 & 299 & 71.6 & 370 \\
\hline Nevada ……….............. & 2,000 & 1,870 & 94 & 151 & 478 & 629 & 169 & 536 & 705 \\
\hline New Hampshire ... & 1,240 & 756 & 61 & 33.0 & 64.1 & 97.1 & 37.0 & 71.9 & 109 \\
\hline New Jersey ............ & 8,410 & 7,460 & 89 & 400 & 650 & 1,050 & 449 & 729 & 1,180 \\
\hline New Mexico .......... & 1,820 & 1,460 & 80 & 262 & 33.8 & 296 & 294 & 37.9 & 332 \\
\hline New York ................ & 19,000 & 17,100 & 90 & 583 & 1,980 & 2,570 & 653 & 2,220 & 2,880 \\
\hline North Carolina ..... & 8,050 & 5,350 & 66 & 166 & 779 & 945 & 186 & 873 & 1,060 \\
\hline North Dakota ........ & 642 & 493 & 77 & 32.4 & 31.2 & 63.6 & 36.3 & 35.0 & 71.3 \\
\hline Ohio …………........... & 11,400 & 9,570 & 84 & 500 & 966 & 1,470 & 560 & 1,080 & 1,640 \\
\hline Oklahoma ………... & 3,450 & 3,150 & 91 & 113 & 562 & 675 & 127 & 631 & 757 \\
\hline Oregon …………........ & 3,420 & 2,730 & 80 & 118 & 447 & 566 & 133 & 501 & 634 \\
\hline Pennsylvania .......... & 12,300 & 10,100 & 82 & 212 & 1,250 & 1,460 & 237 & 1,400 & 1,640 \\
\hline Rhode Island ......... & 1,050 & 922 & 88 & 16.9 & 102 & 119 & 19.0 & 115 & 134 \\
\hline South Carolina ..... & 4,010 & 3,160 & 79 & 105 & 462 & 566 & 117 & 517 & 635 \\
\hline South Dakota ........ & 755 & 625 & 83 & 54.2 & 39.1 & 93.3 & 60.7 & 43.9 & 105 \\
\hline Tennessee ................ & 5,690 & 5,240 & 92 & 321 & 569 & 890 & 360 & 638 & 997 \\
\hline Texas ......................... & 20,900 & 19,700 & 94 & 1,260 & 2,970 & 4,230 & 1,420 & 3,330 & 4,740 \\
\hline Utah ......................... & 2,230 & 2,180 & 97 & 364 & 274 & 638 & 408 & 307 & 715 \\
\hline Vermont .................... & 609 & 362 & 59 & 19.5 & 40.6 & 60.1 & 21.8 & 45.6 & 67.4 \\
\hline Virginia ..................... & 7,080 & 5,310 & 75 & 70.7 & 650 & 720 & 79.3 & 728 & 808 \\
\hline Washington ............ & 5,890 & 4,900 & 83 & 464 & 552 & 1,020 & 520 & 619 & 1,140 \\
\hline West Virginia ........ & 1,810 & 1,300 & 72 & 41.6 & 149 & 190 & 46.6 & 167 & 213 \\
\hline Wisconsin ................. & 5,360 & 3,620 & 67 & 330 & 293 & 623 & 370 & 329 & 699 \\
\hline Wyoming ……............ & 494 & 406 & 82 & 57.2 & 49.4 & 107 & 64.1 & 55.3 & 119 \\
\hline Puerto Rico ............ & 3,810 & 3,800 & 100 & 88.5 & 425 & 513 & 99.2 & 476 & 576 \\
\hline U.S. Virgin Islands & 109 & 53.4 & 49 & .52 & 5.57 & 6.09 & .58 & 6.24 & 6.83 \\
\hline TOTAL & 285,000 & 242,000 & 85 & 16,000 & 27,300 & 43,300 & 17,900 & 30,600 & 48,500 \\
\hline
\end{tabular}



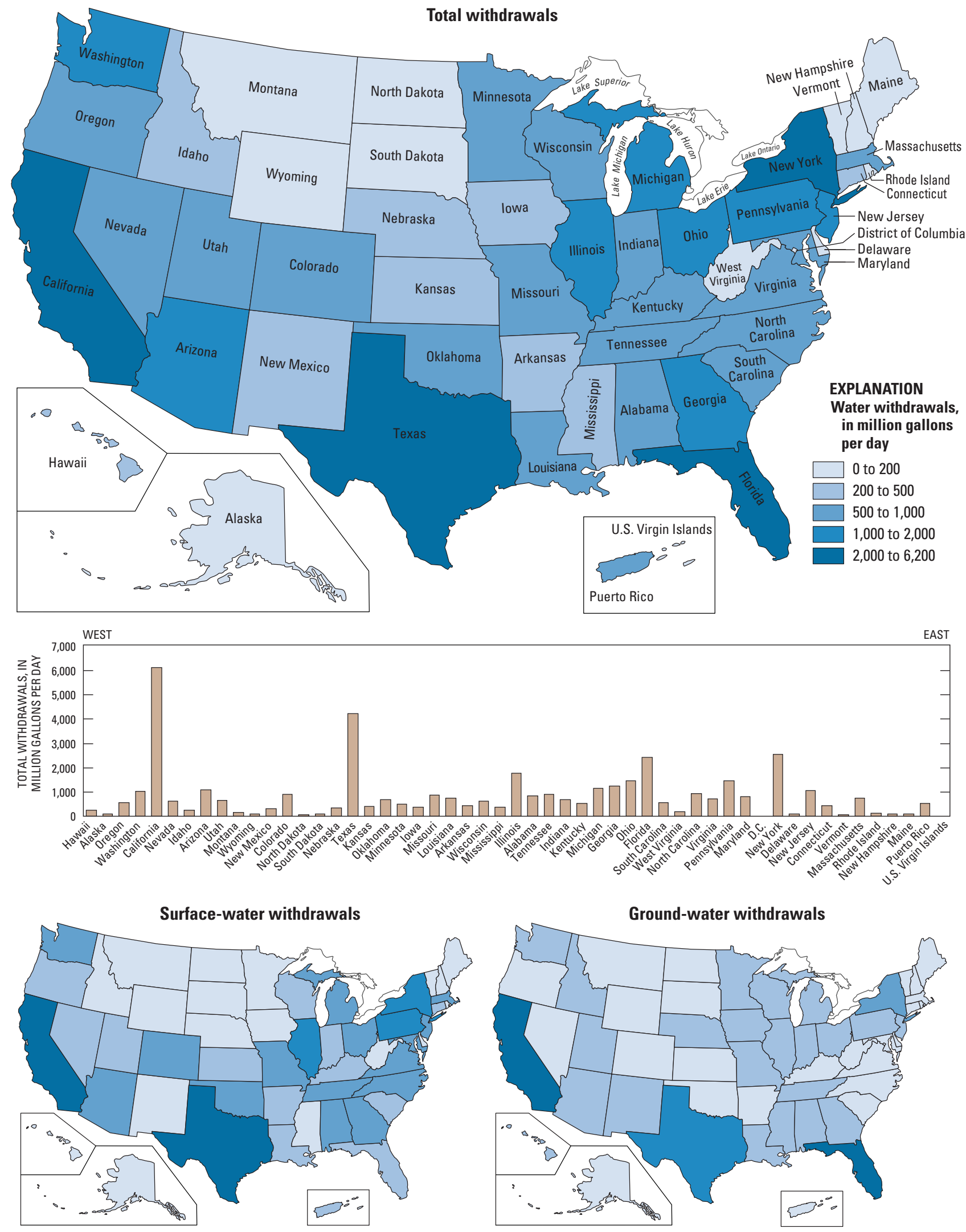

Figure 5. Public-supply withdrawals by source and State, 2000. 
Domestic water use is water used for indoor and outdoor household purposes. Common indoor uses include drinking, preparing food, bathing, washing clothes and dishes, and flushing toilets. The major outdoor uses are watering lawns and gardens. Water for domestic use may be delivered from a public supplier or be self-supplied. In this report, domestic use refers to self-supplied withdrawals only. For selfsupplied domestic water, the source usually is a well. All self-supplied domestic withdrawals were considered freshwater. Self-supplied domestic population was defined as the difference between the total population for 2000, as determined by the U.S. Census Bureau, and the population served by public suppliers. Public-supply deliveries to domestic users and consumptive use were not reported for 2000.

Self-supplied domestic withdrawals and population are listed by State in table 6. For 2000, withdrawals were an estimated 3,590 Mgal/d or 4,030 thousand acre-feet per year. Self-supplied domestic withdrawals were about 1 percent of total freshwater withdrawals and about 2 percent of total freshwater withdrawals for all categories excluding thermoelectric power. About 43.5 million people were self-supplied. Ground water was the primary source of the water (98 percent). Between 1995 and 2000, self-supplied domestic withdrawals increased about 6 percent and the self-supplied domestic population increased about 2 percent. The self-supplied domestic population was 15 percent of the total U.S. population.

The geographic distribution of total withdrawals for self-

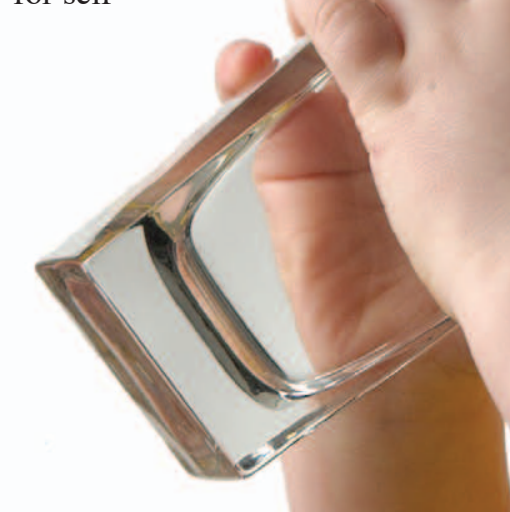

(Photo courtesy of Glenn Phillips, IMEC.) supplied domestic use, self-supplied domestic population, and self-supplied domestic population as a percentage of the State total population is shown in figures $6 \mathrm{a}$ and $6 \mathrm{~b}$, respectively. The largest self-supplied domestic withdrawals and self-supplied populations were in California and Michigan. These two States represented 15 percent of the total selfsupplied domestic withdrawals and 15 percent of the total self-supplied domestic population. Some States, such as the U.S. Virgin Islands, Maine, and Vermont, had large percentages of the total State population that were self-supplied.

Self-supplied domestic withdrawals are rarely measured or reported. Therefore, self-supplied domestic withdrawals were estimated using self-supplied domestic popula-

tion and per capita use coefficients.

Coefficients varied from one geographic region to another. Domestic per capita use coefficients were either derived using public-supply delivery data for domestic use, or were obtained from a State agency, a planning or research organization, or were obtained from published values in the technical literature. 
Table 6. Self-supplied domestic water withdrawals, 2000.

[Figures may not sum to totals because of independent rounding]

\begin{tabular}{|c|c|c|c|c|c|c|c|c|c|c|}
\hline \multirow{3}{*}{ STATE } & \multicolumn{4}{|c|}{$\begin{array}{l}\text { POPULATION } \\
\text { (in thousands) }\end{array}$} & \multicolumn{3}{|c|}{$\begin{array}{c}\text { WITHDRAWALS } \\
\text { (in million gallons per day) }\end{array}$} & \multicolumn{3}{|c|}{$\begin{array}{c}\text { WITHDRAWALS } \\
\text { (in thousand acre-feet per year) }\end{array}$} \\
\hline & \multirow[b]{2}{*}{ Total } & \multirow[b]{2}{*}{$\begin{array}{l}\text { Served by } \\
\text { public supply }\end{array}$} & \multicolumn{2}{|c|}{ Self-supplied domestic } & \multicolumn{2}{|c|}{ By source } & \multirow[b]{2}{*}{ Total } & \multicolumn{2}{|c|}{ By source } & \multirow[b]{2}{*}{ Total } \\
\hline & & & Population & $\begin{array}{c}\text { Population } \\
\text { (in percent) }\end{array}$ & $\begin{array}{l}\text { Ground } \\
\text { water }\end{array}$ & $\begin{array}{c}\text { Surface } \\
\text { water }\end{array}$ & & $\begin{array}{l}\text { Ground } \\
\text { water }\end{array}$ & $\begin{array}{c}\text { Surface } \\
\text { water }\end{array}$ & \\
\hline 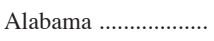 & 4,450 & 3,580 & 868 & 20 & 78.9 & 0 & 78.9 & 88.4 & 0 & 88.4 \\
\hline Alaska ……………....... & 627 & 421 & 206 & 33 & 10.9 & .25 & 11.2 & 12.2 & .28 & 12.5 \\
\hline Arizona ……………...... & 5,130 & 4,870 & 265 & 5 & 28.9 & 0 & 28.9 & 32.4 & 0 & 32.4 \\
\hline Arkansas ..................... & 2,670 & 2,320 & 351 & 13 & 28.5 & 0 & 28.5 & 31.9 & 0 & 31.9 \\
\hline California ……............... & 33,900 & 30,100 & 3,810 & 11 & 257 & 28.6 & 286 & 288 & 32.0 & 320 \\
\hline Colorado ....................... & 4,300 & 3,750 & 555 & 13 & 66.8 & 0 & 66.8 & 74.9 & 0 & 74.9 \\
\hline Connecticut ............... & 3,410 & 2,660 & 749 & 22 & 56.2 & 0 & 56.2 & 63.0 & 0 & 63.0 \\
\hline Delaware ........................ & 784 & 617 & 166 & 21 & 13.3 & 0 & 13.3 & 14.9 & 0 & 14.9 \\
\hline District of Columbia & 572 & 572 & 0 & 0 & 0 & 0 & 0 & 0 & 0 & 0 \\
\hline Florida …….................... & 16,000 & 14,000 & 1,950 & 12 & 199 & 0 & 199 & 223 & 0 & 223 \\
\hline Georgia ……….............. & 8,190 & 6,730 & 1,450 & 18 & 110 & 0 & 110 & 123 & 0 & 123 \\
\hline Hawaii ……….............. & 1,210 & 1,140 & 72.9 & 6 & 4.82 & 7.22 & 12.0 & 5.40 & 8.09 & 13.5 \\
\hline Idaho ............................ & 1,290 & 928 & 366 & 28 & 85.2 & 0 & 85.2 & 95.6 & 0 & 95.6 \\
\hline Illinois ........................ & 12,400 & 10,900 & 1,500 & 12 & 135 & 0 & 135 & 152 & 0 & 152 \\
\hline Indiana …….................. & 6,080 & 4,480 & 1,600 & 26 & 122 & 0 & 122 & 137 & 0 & 137 \\
\hline Iowa …………............ & 2,930 & 2,410 & 511 & 17 & 33.2 & 0 & 33.2 & 37.2 & 0 & 37.2 \\
\hline Kansas ......................... & 2,690 & 2,500 & 193 & 7 & 21.6 & 0 & 21.6 & 24.2 & 0 & 24.2 \\
\hline Kentucky ...................... & 4,040 & 3,490 & 552 & 14 & 19.5 & 8.00 & 27.5 & 21.9 & 8.97 & 30.8 \\
\hline Louisiana ……….......... & 4,470 & 3,950 & 523 & 12 & 41.2 & 0 & 41.2 & 46.2 & 0 & 46.2 \\
\hline Maine ………................. & 1,270 & 726 & 549 & 43 & 35.7 & 0 & 35.7 & 40.0 & 0 & 40.0 \\
\hline Maryland ....................... & 5,300 & 4,360 & 932 & 18 & 77.1 & 0 & 77.1 & 86.4 & 0 & 86.4 \\
\hline Massachusetts .......... & 6,350 & 5,880 & 473 & 7 & 42.2 & 0 & 42.2 & 47.2 & 0 & 47.2 \\
\hline Michigan ....................... & 9,940 & 7,170 & 2,770 & 28 & 239 & 0 & 239 & 268 & 0 & 268 \\
\hline Minnesota ................... & 4,920 & 3,770 & 1,150 & 23 & 80.8 & 0 & 80.8 & 90.6 & 0 & 90.6 \\
\hline Mississippi ...................... & 2,840 & 2,190 & 654 & 23 & 69.3 & 0 & 69.3 & 77.7 & 0 & 77.7 \\
\hline Missouri ………........... & 5,600 & 4,770 & 824 & 15 & 53.6 & 0 & 53.6 & 60.1 & 0 & 60.1 \\
\hline Montana ……….............. & 902 & 664 & 238 & 26 & 17.3 & 1.29 & 18.6 & 19.4 & 1.45 & 20.8 \\
\hline Nebraska ...................... & 1,710 & 1,390 & 324 & 19 & 48.4 & 0 & 48.4 & 54.3 & 0 & 54.3 \\
\hline Nevada ........................ & 2,000 & 1,870 & 124 & 6 & 22.4 & 0 & 22.4 & 25.2 & 0 & 25.2 \\
\hline New Hampshire ....... & 1,240 & 756 & 479 & 39 & 40.9 & .16 & 41.0 & 45.8 & .18 & 46.0 \\
\hline New Jersey ………...... & 8,410 & 7,460 & 952 & 11 & 79.7 & 0 & 79.7 & 89.3 & 0 & 89.3 \\
\hline New Mexico ................. & 1,820 & 1,460 & 360 & 20 & 31.4 & 0 & 31.4 & 35.2 & 0 & 35.2 \\
\hline New York ..................... & 19,000 & 17,100 & 1,890 & 10 & 142 & 0 & 142 & 159 & 0 & 159 \\
\hline North Carolina ......... & 8,050 & 5,350 & 2,700 & 34 & 189 & 0 & 189 & 212 & 0 & 212 \\
\hline North Dakota ............ & 642 & 493 & 149 & 23 & 11.9 & 0 & 11.9 & 13.3 & 0 & 13.3 \\
\hline Ohio .......................... & 11,400 & 9,570 & 1,790 & 16 & 132 & 2.71 & 134 & 148 & 3.04 & 151 \\
\hline Oklahoma ………........ & 3,450 & 3,150 & 299 & 9 & 25.5 & 0 & 25.5 & 28.5 & 0 & 28.5 \\
\hline Oregon …………......... & 3,420 & 2,730 & 692 & 20 & 68.3 & 7.97 & 76.2 & 76.5 & 8.93 & 85.5 \\
\hline Pennsylvania ................. & 12,300 & 10,100 & 2,190 & 18 & 132 & 0 & 132 & 148 & 0 & 148 \\
\hline Rhode Island .............. & 1,050 & 922 & 127 & 12 & 8.99 & 0 & 8.99 & 10.1 & 0 & 10.1 \\
\hline South Carolina .......... & 4,010 & 3,160 & 847 & 21 & 63.5 & 0 & 63.5 & 71.2 & 0 & 71.2 \\
\hline South Dakota ............ & 755 & 625 & 129 & 17 & 9.52 & .01 & 9.53 & 10.7 & .01 & 10.7 \\
\hline Tennessee .................... & 5,690 & 5,240 & 453 & 8 & 32.6 & 0 & 32.6 & 36.6 & 0 & 36.6 \\
\hline 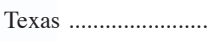 & 20,900 & 19,700 & 1,190 & 6 & 131 & 0 & 131 & 147 & 0 & 147 \\
\hline Utah ........................... & 2,230 & 2,180 & 56.2 & 3 & 16.1 & 0 & 16.1 & 18.0 & 0 & 18.0 \\
\hline Vermont ……............. & 609 & 362 & 247 & 41 & 20.7 & .25 & 21.0 & 23.2 & .28 & 23.5 \\
\hline Virginia ………….......... & 7,080 & 5,310 & 1,770 & 25 & 133 & 0 & 133 & 150 & 0 & 150 \\
\hline Washington ............... & 5,890 & 4,900 & 993 & 17 & 125 & .02 & 125 & 140 & .02 & 140 \\
\hline West Virginia ............ & 1,810 & 1,300 & 505 & 28 & 39.6 & .81 & 40.4 & 44.4 & .91 & 45.3 \\
\hline Wisconsin .................... & 5,360 & 3,620 & 1,750 & 33 & 96.3 & 0 & 96.3 & 108 & 0 & 108 \\
\hline 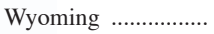 & 494 & 406 & 87.5 & 18 & 6.57 & 0 & 6.57 & 7.36 & 0 & 7.36 \\
\hline Puerto Rico .................. & 3,810 & 3,800 & 12.8 & 0 & .88 & 0 & .88 & .99 & 0 & .99 \\
\hline U.S. Virgin Islands .. & 109 & 53.4 & 55.2 & 51 & 0 & 1.69 & 1.69 & 0 & 1.89 & 1.89 \\
\hline TOTAL & 285,000 & 242,000 & 43,500 & 15 & 3,530 & 58.9 & 3,590 & 3,960 & 66.1 & 4,030 \\
\hline
\end{tabular}



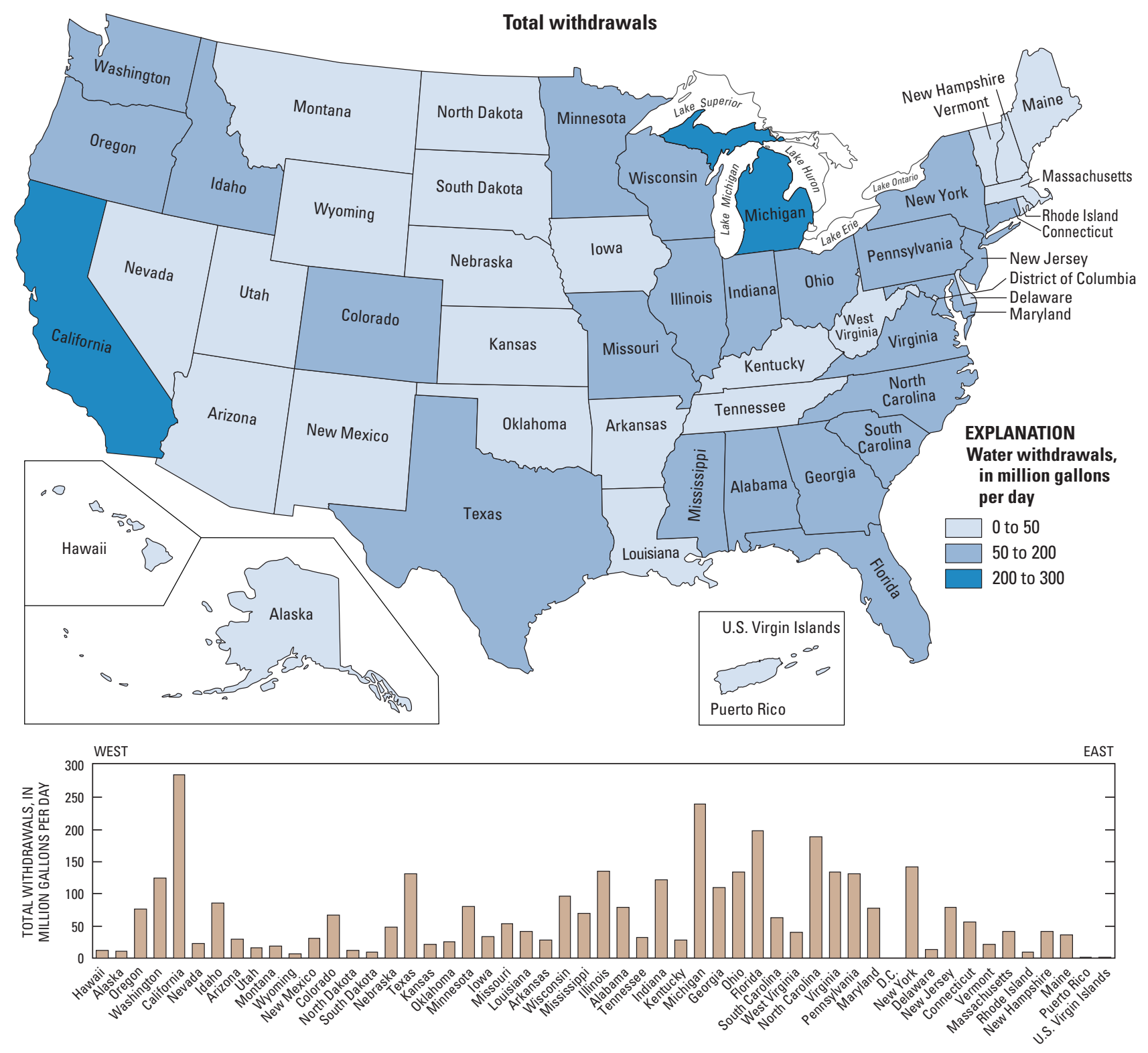

Figure 6a. Self-supplied domestic withdrawals by State, 2000. 

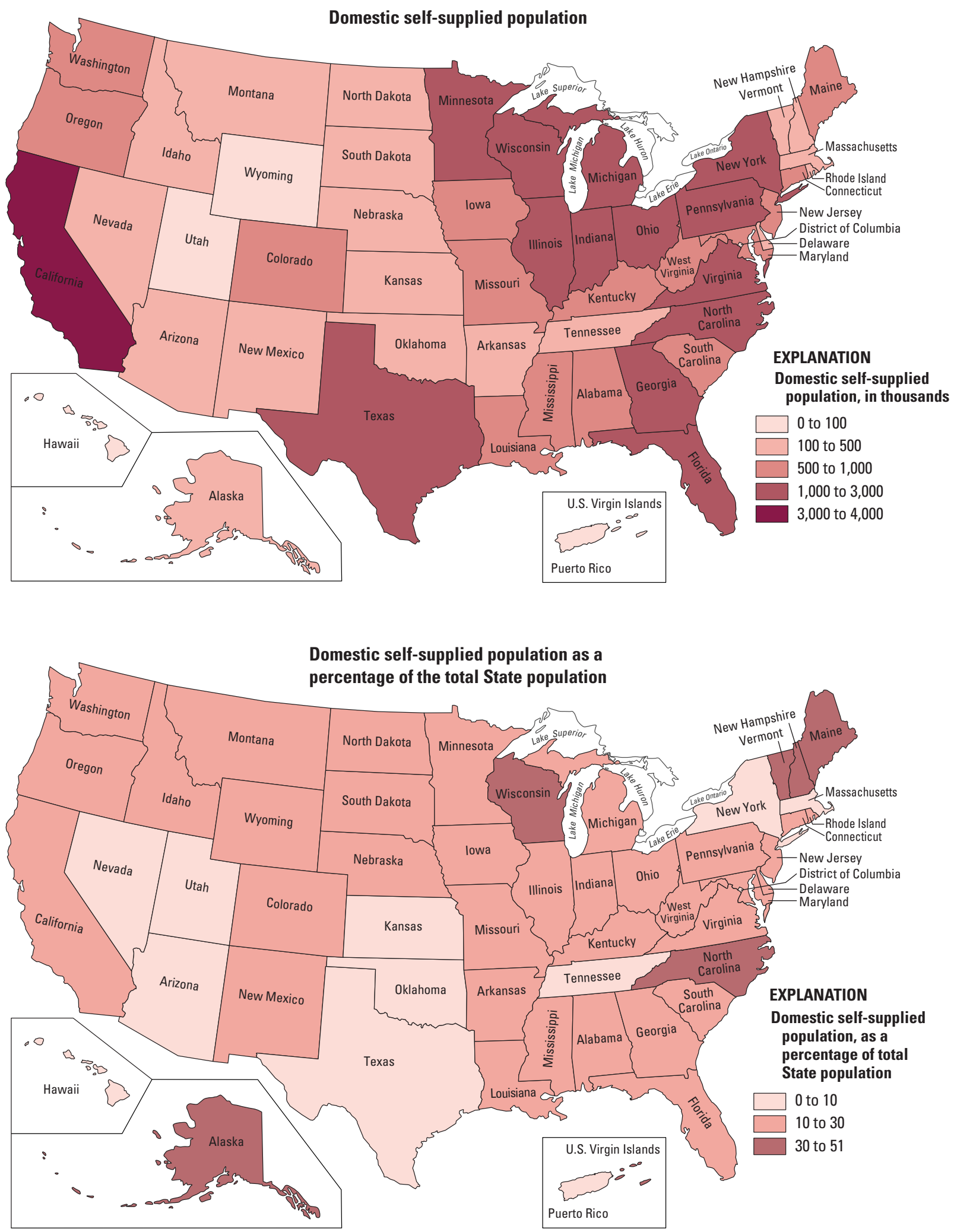

Figure 6b. Self-supplied domestic population and self-supplied domestic population as a percentage of total population, 2000. 
Irrigation water use includes water that is applied by an irrigation system to sustain plant growth in all agricultural and horticultural practices. Irrigation also includes water that is applied for pre-irrigation, frost protection, application of chemicals, weed control, field preparation, crop cooling, harvesting, dust suppression, leaching salts from the root zone, and water lost in conveyance. Irrigation of golf courses, parks, nurseries, turf farms, cemeteries, and other self-supplied landscape-watering uses also are included. Irrigation water use includes self-supplied withdrawals and deliveries from irrigation companies, irrigation districts, cooperatives, or governmental entities. All irrigation withdrawals were considered freshwater. Irrigated acres were reported by three types of irrigation methods: sprinkler, microirrigation, and surface (flood). For 2000, reclaimed wastewater, conveyances losses, and consumptive use were not reported separately for irrigation.

Irrigation withdrawals and irrigated acres by irrigation system are listed by State in table 7. For 2000, withdrawals were an estimated $137,000 \mathrm{Mgal} / \mathrm{d}$, or 153,000 thousand acre-feet per year. Irrigation withdrawals were 40 percent of total freshwater withdrawals and 65 percent of total freshwater withdrawals for all categories excluding thermoelectric power. Surface water accounted for 58 percent of the total irrigation withdrawals. About 61,900 thousand acres were irrigated in 2000. Of this total acreage, about 29,400 thousand acres were irrigated with surface (flood) systems; 28,300 thousand acres with sprinkler systems; and 4,180 thousand acres with microirrigation systems. Application rates were calculated by dividing total withdrawals by irrigated acres. The average application rate was 2.48 acre-feet per acre for the United States.

The geographic distribution of total, surface-water, and ground-water withdrawals for irrigation is shown in figure 7. The majority of withdrawals (86 percent) and irrigated acres (75 percent) were in the 17 conterminous western States. Irrigated acreage in these States were located in areas where average annual precipitation typically is less than 20 inches and is insufficient to support crops without supplemental water. Surface water was the primary source of water in the arid West and the Mountain States. Ground water was the primary source of water in the Central States. California, Idaho, Colorado, and Nebraska combined accounted for one-half of the total irrigation withdrawals. California and
Idaho accounted for 40 percent of surface-water withdrawals. California and Nebraska accounted for one-third of groundwater withdrawals.

California, Nebraska, Texas, Arkansas, and Idaho accounted for 53 percent of total irrigated acreage. California, Nebraska, and Texas accounted for 40 percent of the irrigated acreage using sprinkler and microirrigation systems. California alone accounted for 72 percent of the irrigated acreage by microirrigation systems. Sprinkler and microirrigation systems were associated with slightly more than 50 percent of total irrigated acreage.

Application rates were greatest in the arid West and the Mountain States where surface water was available and surface (flood) application was the predominant method of irrigation. In Arizona, Montana, and Idaho, application rates exceeded 5 acre-feet per acre. States that utilized the High Plains aquifer (Nebraska, Texas, Kansas, and Oklahoma) or the Mississippi River alluvium (Arkansas, Missouri, Mississippi, and Louisiana) for irrigation relied mostly on ground water and had application rates ranging between 1 and 2 acrefeet per acre. Sprinkler irrigation was the predominant application method in the Central Plains States of Kansas, Nebraska, North Dakota, Oklahoma, South Dakota, and Texas. Surface irrigation was the predominant application method in Arkansas, Louisiana, Mississippi, and Missouri.

Estimates of total irrigation withdrawals for 2000 were about 2 percent more than during 1995. Surface-water withdrawals were about 5 percent less for 2000 compared to 1995 , but ground-water withdrawals were 16 percent more. The estimated number of irrigated acres was about 7 percent more, which resulted in a slightly lower average application rate for 2000. The number of acres irrigated for 2000 using sprinkler or microirrigation systems was 25 percent more than during 1995 .

Sources of data for irrigation withdrawals and irrigated acres included State and Federal crop reporting programs. Withdrawals also were estimated using information on irrigated crop acreages along with specific crop waterconsumption coefficients or irrigation-system application rates. Estimation methods varied from one geographic area to another. Estimation methods ideally included adjustments for climatic variables, system efficiencies, conveyance losses, and other irrigation practices such as pre-irrigation. Other methods of estimating irrigation withdrawals included extrapolation of sample data on crop water-application rates or power-consumption coefficients. 
Table 7. Irrigation water withdrawals, 2000.

[Figures may not sum to totals because of independent rounding]

\begin{tabular}{|c|c|c|c|c|c|c|c|c|c|c|c|}
\hline \multirow{3}{*}{ STATE } & \multicolumn{4}{|c|}{$\begin{array}{l}\text { IRRIGATED LAND } \\
\text { (in thousand acres) }\end{array}$} & \multicolumn{3}{|c|}{$\begin{array}{c}\text { WITHDRAWALS } \\
\text { (in million gallons per day) }\end{array}$} & \multicolumn{3}{|c|}{$\begin{array}{l}\text { WITHDRAWALS } \\
\text { (in thousand acre-feet per year) }\end{array}$} & \multirow{3}{*}{$\begin{array}{l}\text { APPLICATION RATE } \\
\text { (in acre-feet } \\
\text { per acre) }\end{array}$} \\
\hline & \multicolumn{3}{|c|}{ By type of irrigation } & \multirow[b]{2}{*}{ Total } & \multicolumn{2}{|c|}{ By source } & \multirow[b]{2}{*}{ Total } & \multicolumn{2}{|c|}{ By source } & \multirow[b]{2}{*}{ Total } & \\
\hline & Sprinkler & $\begin{array}{l}\text { Micro- } \\
\text { irrigation }\end{array}$ & Surface & & $\begin{array}{l}\text { Ground } \\
\text { water }\end{array}$ & $\begin{array}{l}\text { Surface } \\
\text { water }\end{array}$ & & $\begin{array}{l}\text { Ground } \\
\text { water }\end{array}$ & $\begin{array}{l}\text { Surface } \\
\text { water }\end{array}$ & & \\
\hline Alabama ...................... & 68.7 & 1.30 & 0 & 70.0 & 14.5 & 28.7 & 43.1 & 16.2 & 32.2 & 48.4 & 0.69 \\
\hline 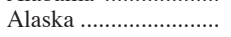 & 2.43 & 0 & .07 & 2.50 & .99 & .02 & 1.01 & 1.11 & .02 & 1.13 & .45 \\
\hline Arizona .......................... & 183 & 14.0 & 779 & 976 & 2,750 & 2,660 & 5,400 & 3,080 & 2,980 & 6,060 & 6.21 \\
\hline Arkansas ........................ & 631 & 0 & 3,880 & 4,510 & 6,510 & 1,410 & 7,910 & 7,290 & 1,580 & 8,870 & 1.97 \\
\hline California ...................... & 1,660 & 3,010 & 5,470 & 10,100 & 11,600 & 18,900 & 30,500 & 13,100 & 21,100 & 34,200 & 3.37 \\
\hline Colorado ......................... & 1,190 & 1.16 & 2,220 & 3,400 & 2,160 & 9,260 & 11,400 & 2,420 & 10,400 & 12,800 & 3.76 \\
\hline Connecticut ................. & 20.6 & .39 & 0 & 21.0 & 17.0 & 13.4 & 30.4 & 19.0 & 15.0 & 34.0 & 1.62 \\
\hline Delaware ....................... & 81.1 & .71 & 0 & 81.8 & 35.6 & 7.89 & 43.5 & 39.9 & 8.84 & 48.7 & .60 \\
\hline District of Columbia . & .32 & 0 & 0 & .32 & 0 & .18 & .18 & 0 & .20 & .20 & .63 \\
\hline Florida …....................... & 515 & 704 & 839 & 2,060 & 2,180 & 2,110 & 4,290 & 2,450 & 2,370 & 4,810 & 2.34 \\
\hline Georgia .......................... & 1,470 & 73.8 & 0 & 1,540 & 750 & 392 & 1,140 & 841 & 439 & 1,280 & .83 \\
\hline 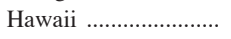 & 16.7 & 105 & 0 & 122 & 171 & 193 & 364 & 191 & 216 & 407 & 3.35 \\
\hline 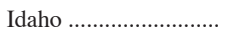 & 2,440 & 4.70 & 1,300 & 3,750 & 3,720 & 13,300 & 17,100 & 4,170 & 15,000 & 19,100 & 5.10 \\
\hline 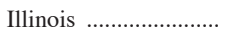 & 365 & 0 & 0 & 365 & 150 & 4.25 & 154 & 168 & 4.76 & 173 & .47 \\
\hline Indiana & 250 & 0 & 0 & 250 & 55.5 & 45.4 & 101 & 62.2 & 51.0 & 113 & .45 \\
\hline 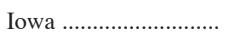 & 84.5 & 0 & 0 & 84.5 & 20.4 & 1.08 & 21.5 & 22.9 & 1.21 & 24.1 & .28 \\
\hline Kansas ............................ & 2,660 & 2.14 & 647 & 3,310 & 3,430 & 288 & 3,710 & 3,840 & 323 & 4,160 & 1.26 \\
\hline Kentucky ....................... & 66.6 & 0 & 0 & 66.6 & 1.14 & 28.2 & 29.3 & 1.28 & 31.6 & 32.9 & .49 \\
\hline Louisiana ....................... & 110 & 0 & 830 & 940 & 791 & 232 & 1,020 & 887 & 261 & 1,150 & 1.22 \\
\hline Maine ........................... & 35.0 & .95 & .03 & 36.0 & .61 & 5.23 & 5.84 & .68 & 5.86 & 6.55 & .18 \\
\hline Maryland ........................ & 57.3 & 3.32 & 0 & 60.6 & 29.8 & 12.6 & 42.4 & 33.4 & 14.1 & 47.6 & .78 \\
\hline Massachusetts ............ & 26.6 & 2.35 & 0 & 29.0 & 19.7 & 106 & 126 & 22.1 & 119 & 141 & 4.88 \\
\hline Michigan ..................... & 401 & 8.67 & 4.87 & 415 & 128 & 73.2 & 201 & 144 & 82.0 & 226 & .54 \\
\hline Minnesota ...................... & 546 & 0 & 26.9 & 573 & 190 & 36.6 & 227 & 213 & 41.1 & 254 & .44 \\
\hline Mississippi ................ & 455 & 0 & 966 & 1,420 & 1,310 & 99.1 & 1,410 & 1,470 & 111 & 1,580 & 1.11 \\
\hline Missouri ….................... & 532 & 1.43 & 792 & 1,330 & 1,380 & 48.1 & 1,430 & 1,550 & 53.9 & 1,600 & 1.21 \\
\hline Montana ........................ & 506 & 0 & 1,220 & 1,720 & 83.0 & 7,870 & 7,950 & 93.0 & 8,820 & 8,920 & 5.18 \\
\hline Nebraska ........................ & 4,110 & 0 & 3,710 & 7,820 & 7,420 & 1,370 & 8,790 & 8,320 & 1,540 & 9,860 & 1.26 \\
\hline Nevada ........................ & 192 & 0 & 456 & 647 & 567 & 1,540 & 2,110 & 635 & 1,730 & 2,360 & 3.65 \\
\hline New Hampshire .......... & 6.08 & 0 & 0 & 6.08 & .50 & 4.25 & 4.75 & .56 & 4.76 & 5.32 & .88 \\
\hline New Jersey ................... & 109 & 15.7 & 3.70 & 128 & 22.8 & 117 & 140 & 25.5 & 131 & 156 & 1.22 \\
\hline New Mexico ............... & 461 & 7.17 & 530 & 998 & 1,230 & 1,630 & 2,860 & 1,380 & 1,830 & 3,210 & 3.22 \\
\hline New York ..................... & 70.0 & 8.73 & 1.84 & 80.6 & 23.3 & 12.1 & 35.5 & 26.1 & 13.6 & 39.8 & .49 \\
\hline North Carolina ........... & 193 & 3.70 & 0 & 196 & 65.8 & 221 & 287 & 73.8 & 248 & 322 & 1.64 \\
\hline North Dakota ............. & 200 & 0 & 26.7 & 227 & 72.2 & 73.2 & 145 & 80.9 & 82.1 & 163 & .72 \\
\hline 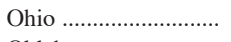 & 61.0 & 0 & 0 & 61.0 & 13.9 & 17.8 & 31.7 & 15.6 & 19.9 & 35.5 & .58 \\
\hline Oklahoma ...................... & 392 & 1.50 & 113 & 507 & 566 & 151 & 718 & 635 & 170 & 804 & 1.59 \\
\hline Oregon …....................... & 1,160 & 4.02 & 1,000 & 2,170 & 792 & 5,290 & 6,080 & 887 & 5,920 & 6,810 & 3.14 \\
\hline Pennsylvania ............. & 28.9 & 7.17 & 0 & 36.0 & 1.38 & 12.5 & 13.9 & 1.55 & 14.0 & 15.6 & .43 \\
\hline Rhode Island ............. & 4.48 & .29 & .05 & 4.82 & .46 & 2.99 & 3.45 & .52 & 3.35 & 3.87 & .80 \\
\hline South Carolina ........... & 166 & 3.66 & 17.5 & 187 & 106 & 162 & 267 & 118 & 181 & 300 & 1.60 \\
\hline South Dakota .............. & 276 & 0 & 78.3 & 354 & 137 & 236 & 373 & 153 & 264 & 418 & 1.18 \\
\hline Tennessee .................... & 51.2 & 5.35 & 3.96 & 60.5 & 7.33 & 15.1 & 22.4 & 8.22 & 16.9 & 25.1 & .41 \\
\hline 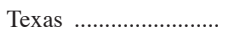 & 4,010 & 89.4 & 2,390 & 6,490 & 6,500 & 2,130 & 8,630 & 7,290 & 2,390 & 9,680 & 1.49 \\
\hline 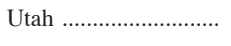 & 526 & 1.68 & 880 & 1,410 & 469 & 3,390 & 3,860 & 526 & 3,800 & 4,330 & 3.08 \\
\hline Vermont ........................ & 4.95 & 0 & 0 & 4.95 & .33 & 3.45 & 3.78 & .37 & 3.87 & 4.24 & .86 \\
\hline Virginia ........................ & 64.3 & 13.9 & 0 & 78.2 & 3.57 & 22.8 & 26.4 & 4.00 & 25.6 & 29.6 & .38 \\
\hline Washington ................... & 1,270 & 49.9 & 252 & 1,570 & 747 & 2,290 & 3,040 & 837 & 2,570 & 3,400 & 2.16 \\
\hline West Virginia .............. & 2.21 & 0 & .98 & 3.19 & .02 & .02 & .04 & .02 & .02 & .04 & .01 \\
\hline Wisconsin ....................... & 355 & 0 & 0 & 355 & 195 & 1.57 & 196 & 218 & 1.76 & 220 & .62 \\
\hline Wyoming ...................... & 190 & 4.73 & 964 & 1,160 & 413 & 4,090 & 4,500 & 463 & 4,580 & 5,050 & 4.36 \\
\hline Puerto Rico ................. & 15.5 & 33.0 & 5.35 & 53.8 & 36.9 & 57.5 & 94.5 & 41.4 & 64.5 & 106 & 1.97 \\
\hline U.S. Virgin Islands ... & .20 & 0 & 0 & .20 & .29 & .21 & .50 & .33 & .24 & .56 & 2.80 \\
\hline TOTAL & 28,300 & 4,180 & 29,400 & 61,900 & 56,900 & 80,000 & 137,000 & 63,800 & 89,700 & 153,000 & 2.48 \\
\hline
\end{tabular}



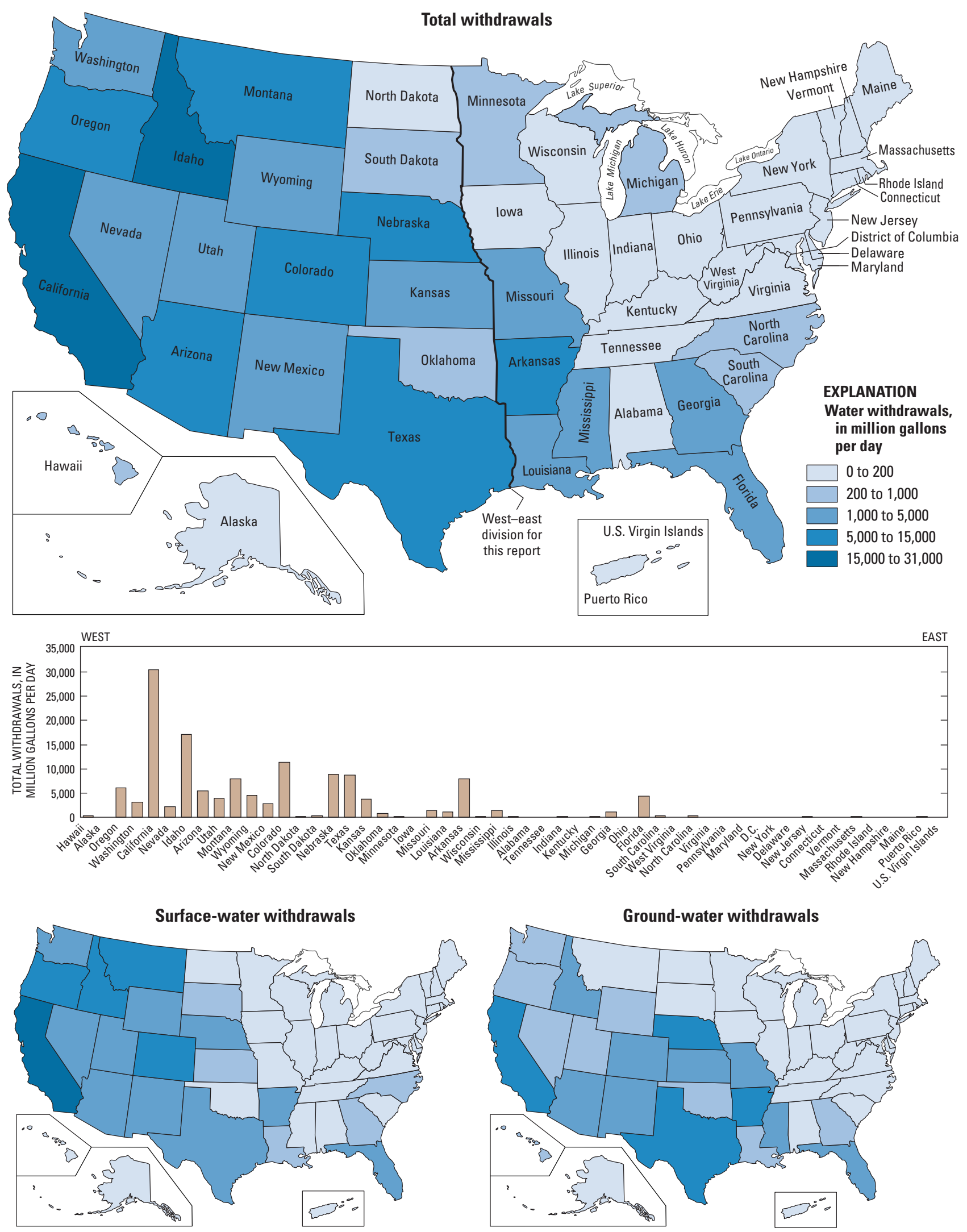

Figure 7. Irrigation withdrawals by source and State, 2000. 
Livestock water use is water associated with livestock watering, feedlots, dairy operations, and other on-farm needs. Livestock includes dairy cows and heifers, beef cattle and calves, sheep and lambs, goats, hogs and pigs, horses, and poultry. Other livestock water uses include cooling of facilities for the animals and products, dairy sanitation and wash down of facilities, animal waste-disposal systems, and incidental water losses. All withdrawals were considered freshwater and self-supplied. The livestock category excludes on-farm domestic use, lawn and garden watering, and irrigation water use. For 2000, consumptive use was not reported for livestock.

In the 1990 and 1995 USGS wateruse Circulars (Solley and others, 1993, 1998, respectively), the livestock category was split into the subcategories of livestock and animal specialties as specified in the 1987 Standard Industrial Classification manual (Office of Management and Budget, 1987). In the 1990 and 1995 Circulars, withdrawals for fish farms and watering horses were included

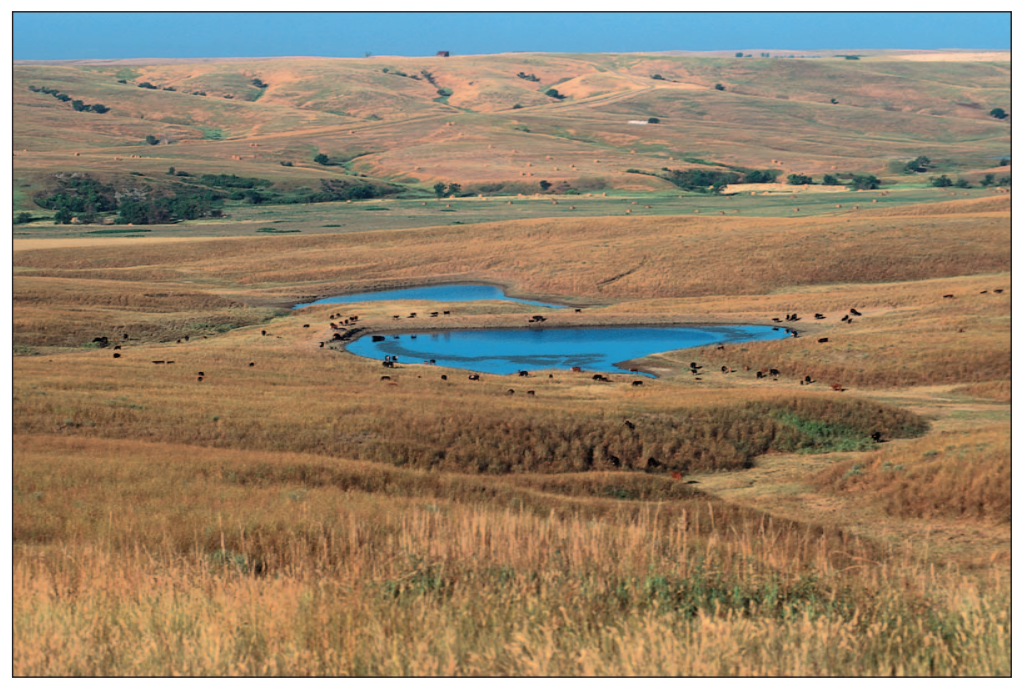

Ponds to deliver water to cattle are important to ranches in South Dakota. (Photo courtesy of USDA Natural Resources Conservation Service.)
Livestock withdrawals for 2000 are listed by State in table 8. During 2000, withdrawals were an estimated $1,760 \mathrm{Mgal} / \mathrm{d}$, or 1,980 thousand acre-feet per year. Livestock withdrawals were less than 1 percent of total freshwater withdrawals and nearly 1 percent of total freshwater withdrawals for all categories excluding thermoelectric power. Ground water was the source for 58 percent of total livestock withdrawals. Total withdrawals for livestock increased slightly for the 22 States that reported data for both 1995 and 2000. However, withdrawals actually increased only in 8 of the 22 reporting States.

The geographic distribution of total, surface-water, and ground-water withdrawals for livestock is shown in figure 8. California, Texas, and Oklahoma accounted for 49 percent of total livestock withdrawals for States that reported for 2000 . California, Texas, North Carolina, and Kansas accounted for nearly one-half of ground-water withdrawals. California, Texas, and Oklahoma accounted for two-thirds of surface-water withdrawals. The areas of major water withdrawals for livestock (as shown in figure 8), for the most part, correspond to the major areas where corn, hay, and alfalfa are grown. For example, the major hog- and pig-producing States of Iowa, Minnesota, and Illinois also are major corn-growing States; the major cattle- and calf-producing States of Texas, Kansas, and Nebraska also are major hay and alfalfa growing States (U.S. Department of Agriculture, May 1999).

Few State agencies require livestock operations to report water withdrawals; therefore, most estimates of withdrawals for livestock typically were derived using animal population counts (most often from the USDA National Agricultural Statistics Service) and a coefficient for gallons of water per animal per day. Coefficients vary by State and, for most States, were provided by agricultural extension agents or water-permitting agencies. Coefficients may reflect both the effect of climate on animal watering and facility maintenance needs. 
Table 8. Livestock water withdrawals, 2000.

[Figures may not sum to totals because of independent rounding. - , data not collected]

\begin{tabular}{|c|c|c|c|c|c|c|}
\hline \multirow{3}{*}{ STATE } & \multicolumn{3}{|c|}{$\begin{array}{c}\text { WITHDRAWALS } \\
\text { (in million gallons per day) }\end{array}$} & \multicolumn{3}{|c|}{$\begin{array}{c}\text { WITHDRAWALS } \\
\text { (in thousand acre-feet per year) }\end{array}$} \\
\hline & \multicolumn{2}{|c|}{ By source } & \multirow[b]{2}{*}{ Total } & \multicolumn{2}{|c|}{ By source } & \multirow[b]{2}{*}{ Total } \\
\hline & $\begin{array}{l}\text { Ground } \\
\text { water }\end{array}$ & $\begin{array}{l}\text { Surface } \\
\text { water }\end{array}$ & & $\begin{array}{l}\text { Ground } \\
\text { water }\end{array}$ & $\begin{array}{l}\text { Surface } \\
\text { water }\end{array}$ & \\
\hline Alabama ...................... & - & - & - & - & - & - \\
\hline Alaska ............................... & - & - & - & - & - & - \\
\hline Arizona …………............ & - & - & - & - & - & - \\
\hline Arkansas ........................ & - & - & - & - & - & - \\
\hline California ..................... & 182 & 227 & 409 & 204 & 255 & 458 \\
\hline Colorado ……................... & - & - & - & - & - & - \\
\hline Connecticut ................ & - & - & - & - & - & - \\
\hline Delaware ....................... & 3.70 & .22 & 3.92 & 4.15 & .25 & 4.39 \\
\hline District of Columbia & - & - & - & - & - & - \\
\hline Florida ……..................... & 31.0 & 1.51 & 32.5 & 34.7 & 1.69 & 36.4 \\
\hline Georgia ............................. & 1.66 & 17.7 & 19.4 & 1.86 & 19.9 & 21.7 \\
\hline Hawaii ............................ & - & - & - & - & - & - \\
\hline Idaho ……...................... & 27.7 & 7.20 & 34.9 & 31.0 & 8.07 & 39.1 \\
\hline Illinois ........................... & 37.6 & 0 & 37.6 & 42.1 & 0 & 42.1 \\
\hline Indiana & 27.3 & 14.6 & 41.9 & 30.6 & 16.4 & 47.0 \\
\hline Iowa …………............... & 81.8 & 27.1 & 109 & 91.8 & 30.4 & 122 \\
\hline Kansas ............................. & 87.2 & 23.5 & 111 & 97.7 & 26.3 & 124 \\
\hline Kentucky .................... & - & - & - & - & - & - \\
\hline Louisiana ........................ & 4.03 & 3.31 & 7.34 & 4.52 & 3.71 & 8.23 \\
\hline Maine …...................... & - & - & - & - & - & - \\
\hline Maryland ...................... & 7.18 & 3.18 & 10.4 & 8.05 & 3.56 & 11.6 \\
\hline Massachusetts ............ & - & - & - & - & - & - \\
\hline Michigan ........................ & 10.2 & 1.15 & 11.3 & 11.4 & 1.29 & 12.7 \\
\hline Minnesota ...................... & 52.8 & 0 & 52.8 & 59.2 & 0 & 59.2 \\
\hline Mississippi ……............. & - & - & - & - & - & - \\
\hline Missouri ……................. & 18.3 & 54.1 & 72.4 & 20.5 & 60.6 & 81.1 \\
\hline Montana ....................... & - & - & - & - & - & - \\
\hline Nebraska ......................... & 76.0 & 17.4 & 93.4 & 85.2 & 19.5 & 105 \\
\hline Nevada ............................ & - & - & - & - & - & - \\
\hline New Hampshire ........ & - & - & - & - & - & - \\
\hline New Jersey ...................... & 1.68 & 0 & 1.68 & 1.88 & 0 & 1.88 \\
\hline New Mexico ............... & - & - & - & - & - & - \\
\hline New York ...................... & - & - & - & - & - & - \\
\hline North Carolina ........... & 89.1 & 32.3 & 121 & 99.9 & 36.2 & 136 \\
\hline North Dakota ............. & - & - & - & - & - & - \\
\hline Ohio ……........................... & 8.20 & 17.1 & 25.3 & 9.19 & 19.2 & 28.4 \\
\hline Oklahoma ....................... & 53.6 & 97.2 & 151 & 60.0 & 109 & 169 \\
\hline Oregon ………................. & - & - & - & - & - & - \\
\hline Pennsylvania ............ & - & - & - & - & - & - \\
\hline Rhode Island ............... & - & - & - & - & - & - \\
\hline South Carolina ........... & - & - & - & - & - & - \\
\hline South Dakota ............. & 16.9 & 25.2 & 42.0 & 18.9 & 28.2 & 47.1 \\
\hline Tennessee ................... & - & - & - & - & - & - \\
\hline Texas ….......................... & 137 & 172 & 308 & 153 & 192 & 346 \\
\hline Utah .............................. & - & - & - & - & - & - \\
\hline Vermont ......................... & - & - & - & - & - & - \\
\hline Virginia ........................... & - & - & - & - & - & - \\
\hline Washington .................... & - & - & - & - & - & - \\
\hline West Virginia ............. & - & - & - & - & - & - \\
\hline Wisconsin ...................... & 60.3 & 6.02 & 66.3 & 67.6 & 6.75 & 74.4 \\
\hline Wyoming ......................... & - & - & - & - & - & - \\
\hline Puerto Rico ................. & - & - & - & - & - & - \\
\hline U.S. Virgin Islands .. & - & - & - & - & - & - \\
\hline TOTAL & 1,010 & 747 & 1,760 & 1,140 & 838 & 1,980 \\
\hline
\end{tabular}



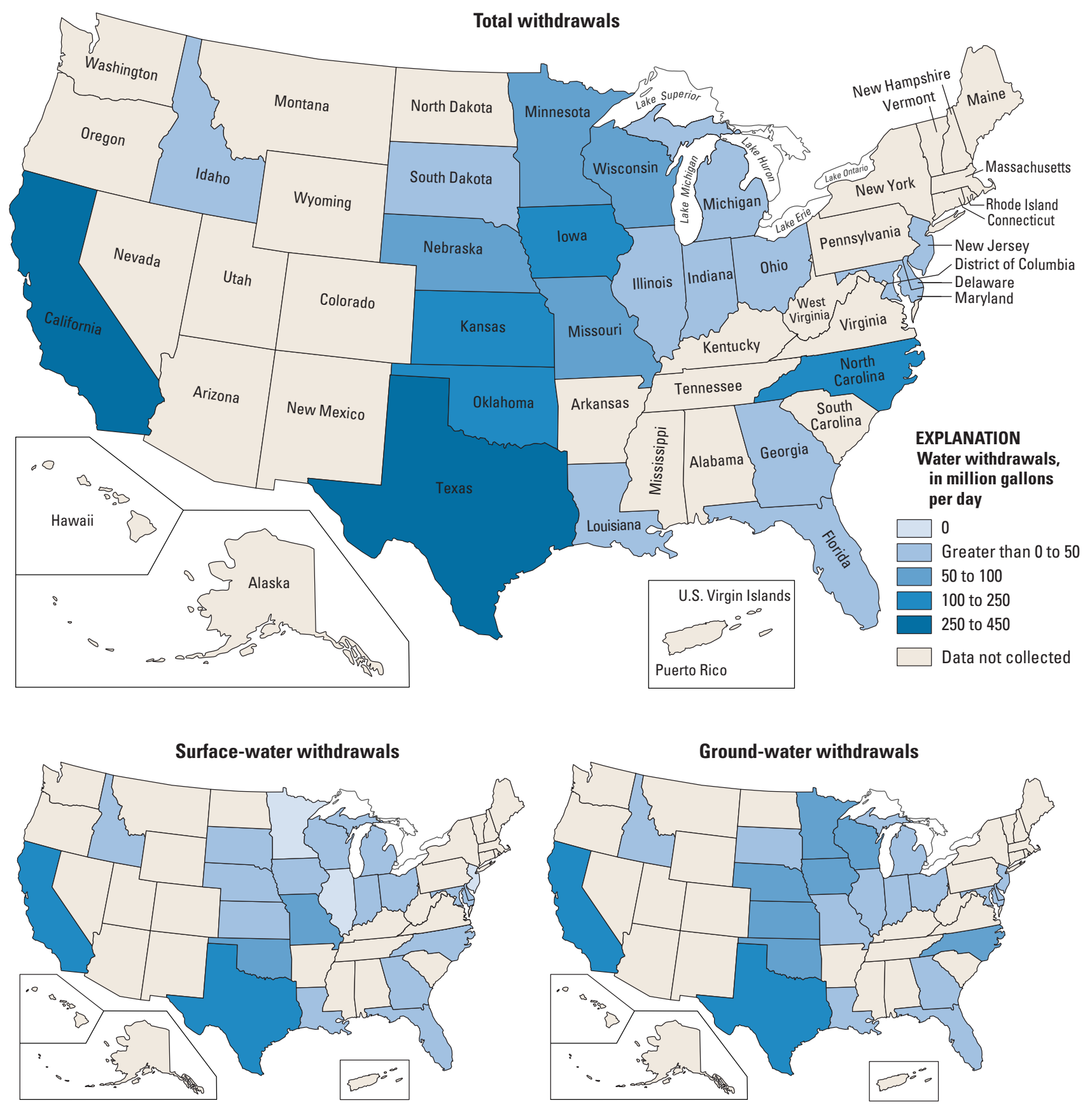

Figure 8. Livestock withdrawals by source and State, 2000. 
Aquaculture water use is water associated with raising organisms that live in water-such as finfish and shellfishfor food, restoration, conservation, or sport. Aquaculture production occurs under controlled feeding, sanitation, and harvesting procedures primarily in ponds, flow-through raceways, and, to a lesser extent, cages, net pens, and closed-recirculation tanks. All withdrawals were considered self-supplied. Only freshwater withdrawals were compiled as part of the total. Aquaculture combines the fishfarming activities of the former livestock subcategory animal specialties and the fish-hatchery activities of the commercial category that were reported during 1990 and 1995 (Solley and others, 1993, 1998, respectively). For 2000 , consumptive use was not reported for aquaculture.

For 2000, the estimate of aquaculture water use for the United States was based on estimates of freshwater withdrawals for aquaculture in 19 States, rather than on estimates from all States. These 19 States included the 8 States with the largest water withdrawals for animal specialties in 1995, and 11 other States in which aquaculture water-use data were collected as part of a broader State water-use program for 2000. Most of the water withdrawals for animal specialties during 1995 were for aquaculture, with a small amount of water primarily used for watering horses. The 19 States that reported for 2000 accounted for 94 percent of the total withdrawals for animal-specialties water use during 1995. During 1995, withdrawals for animal specialties accounted for a small percentage of the total water use, less than 1 percent of the total withdrawals for all categories.
Freshwater withdrawals for aquaculture for 2000 are listed by State in table 9. For 2000, the quantity of freshwater withdrawn for aquaculture was an estimated 3,700 Mgal/d, or 4,150 thousand acre-feet per year. Maryland reported saline withdrawals of $3.09 \mathrm{Mgal} / \mathrm{d}$, which are not listed in the tables or included in the totals. Surface water was the source for about 71 percent of the withdrawals for this category. Aquaculture withdrawals were nearly 1 percent of total water withdrawals and nearly 2 percent of total withdrawals for all categories excluding thermoelectric power.

The geographic distribution of total, surface-water, and ground-water withdrawals for aquaculture is shown in figure 9. Idaho used the most water for aquaculture, about one-half of the total reported. Idaho's source of water was almost exclusively surface water, and represented 73 percent of the total surface-water withdrawals for aquaculture. Mississippi, Arkansas, California, Louisiana, and Utah combined accounted for 86 percent of the ground-water withdrawals for aquaculture.

Several sources of information were used to estimate withdrawals for aquaculture. Some estimates of aquaculture water use were derived from State permits that reported water withdrawals or return flows for aquaculture facilities. The USEPA Permit Compliance System database also was a source of return-flow data that were used to estimate water withdrawals. The State Offices of the USDA National Agricultural Statistics Service or the Cooperative Extension Service sometimes maintained records for a State on pond acreage for fish farms and sometimes on the rate of water lost to evaporation for the ponds.

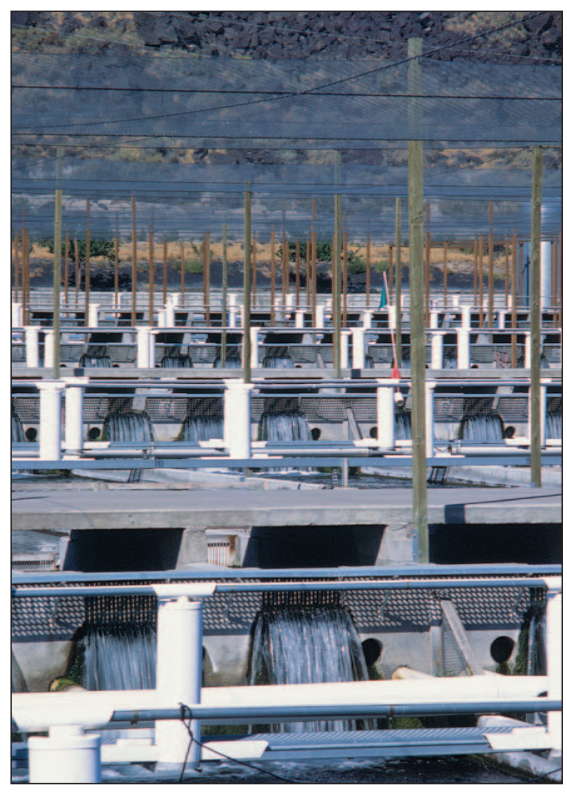

Flow-through raceways are used to raise fish for commercial sale in Idaho. (Photo courtesy of Clear Springs Foods, Inc.)

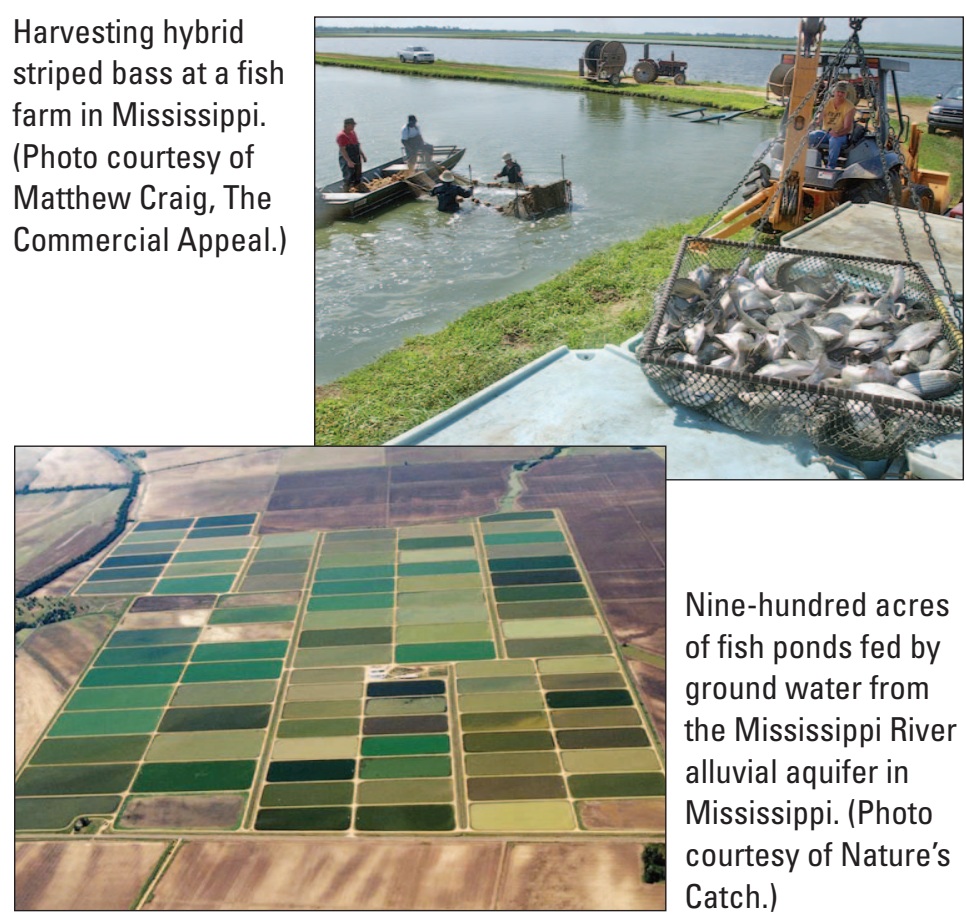

Harvesting hybrid farm in Mississippi. (Photo courtesy of Matthew Craig, The Commercial Appeal. Nine-hundred acres of fish ponds fed by ground water from the Mississippi River alluvial aquifer in Mississippi. (Photo Catch.) 
Table 9. Aquaculture water withdrawals, 2000.

[Figures may not sum to totals because of independent rounding. - , data not collected]

\begin{tabular}{|c|c|c|c|c|c|c|}
\hline \multirow{3}{*}{ STATE } & \multicolumn{3}{|c|}{$\begin{array}{c}\text { WITHDRAWALS } \\
\text { (in million gallons per day) }\end{array}$} & \multicolumn{3}{|c|}{$\begin{array}{c}\text { WITHDRAWALS } \\
\text { (in thousand acre-feet per year) }\end{array}$} \\
\hline & \multicolumn{2}{|c|}{ By source } & \multirow[b]{2}{*}{ Total } & \multicolumn{2}{|c|}{ By source } & \multirow[b]{2}{*}{ Total } \\
\hline & $\begin{array}{l}\text { Ground } \\
\text { water }\end{array}$ & $\begin{array}{c}\text { Surface } \\
\text { water }\end{array}$ & & $\begin{array}{l}\text { Ground } \\
\text { water }\end{array}$ & $\begin{array}{c}\text { Surface } \\
\text { water }\end{array}$ & \\
\hline Alabama …………........... & 8.93 & 1.44 & 10.4 & 10.0 & 1.61 & 11.6 \\
\hline Alaska .............................. & - & - & - & - & - & - \\
\hline Arizona …………............ & - & - & - & - & - & - \\
\hline Arkansas ....................... & 187 & 10.4 & 198 & 210 & 11.6 & 222 \\
\hline California ……............... & 158 & 380 & 537 & 177 & 426 & 603 \\
\hline Colorado ……................. & - & - & - & - & - & - \\
\hline Connecticut ............... & - & - & - & - & - & - \\
\hline Delaware .................... & .07 & 0 & .07 & .08 & 0 & .08 \\
\hline District of Columbia & - & - & - & - & - & - \\
\hline Florida …….................... & 7.81 & .21 & 8.02 & 8.76 & .24 & 8.99 \\
\hline Georgia ……..................... & 7.70 & 7.72 & 15.4 & 8.63 & 8.65 & 17.3 \\
\hline Hawaii .............................. & - & - & - & - & - & - \\
\hline Idaho ............................... & 51.5 & 1,920 & 1,970 & 57.7 & 2,150 & 2,210 \\
\hline Illinois ........................ & - & - & - & - & - & - \\
\hline Indiana .......................... & - & - & - & - & - & - \\
\hline Iowa ................................. & - & - & - & - & - & - \\
\hline Kansas ............................. & 3.33 & 2.27 & 5.60 & 3.73 & 2.54 & 6.28 \\
\hline Kentucky ...................... & - & - & - & - & - & - \\
\hline Louisiana ........................ & 128 & 115 & 243 & 144 & 129 & 273 \\
\hline Maine ……...................... & - & - & - & - & - & - \\
\hline Maryland ................... & 4.81 & 14.8 & 19.6 & 5.39 & 16.6 & 22.0 \\
\hline Massachusetts ........... & - & - & - & - & - & - \\
\hline Michigan ...................... & - & - & - & - & - & - \\
\hline Minnesota ..................... & - & - & - & - & - & - \\
\hline Mississippi .................. & 321 & 49.8 & 371 & 360 & 55.9 & 416 \\
\hline Missouri ………............. & 2.01 & 81.3 & 83.3 & 2.25 & 91.2 & 93.4 \\
\hline Montana ......................... & - & - & - & - & - & - \\
\hline Nebraska ......................... & - & - & - & - & - & - \\
\hline Nevada …………............ & - & - & - & - & - & - \\
\hline New Hampshire ........ & 3.12 & 13.1 & 16.3 & 3.50 & 14.7 & 18.2 \\
\hline New Jersey ................ & 6.46 & 0 & 6.46 & 7.24 & 0 & 7.24 \\
\hline New Mexico .............. & - & - & - & - & - & - \\
\hline New York ..................... & - & - & - & - & - & - \\
\hline North Carolina ........... & 7.88 & 0 & 7.88 & 8.83 & 0 & 8.83 \\
\hline North Dakota ................ & - & - & - & - & - & - \\
\hline Ohio ……........................ & 1.36 & 0 & 1.36 & 1.52 & 0 & 1.52 \\
\hline Oklahoma ...................... & .29 & 16.1 & 16.4 & .33 & 18.1 & 18.4 \\
\hline Oregon ........................... & - & - & - & - & - & - \\
\hline Pennsylvania ............... & - & - & - & - & - & - \\
\hline Rhode Island ............ & - & - & - & - & - & - \\
\hline South Carolina ........... & - & - & - & - & - & - \\
\hline South Dakota ............. & - & - & - & - & - & - \\
\hline Tennessee …….............. & - & - & - & - & - & - \\
\hline Texas …….................... & - & - & - & - & - & - \\
\hline Utah ................................. & 116 & 0 & 116 & 130 & 0 & 130 \\
\hline Vermont ......................... & - & - & - & - & - & - \\
\hline Virginia ......................... & - & - & - & - & - & - \\
\hline Washington ................. & - & - & - & - & - & - \\
\hline West Virginia ............. & - & - & - & - & - & - \\
\hline Wisconsin ...................... & 39.8 & 30.4 & 70.2 & 44.6 & 34.1 & 78.7 \\
\hline Wyoming ........................ & - & - & - & - & - & - \\
\hline Puerto Rico ................. & - & - & - & - & - & - \\
\hline U.S. Virgin Islands .. & - & - & - & - & - & - \\
\hline TOTAL & 1,060 & 2,640 & 3,700 & 1,180 & 2,960 & 4,150 \\
\hline
\end{tabular}



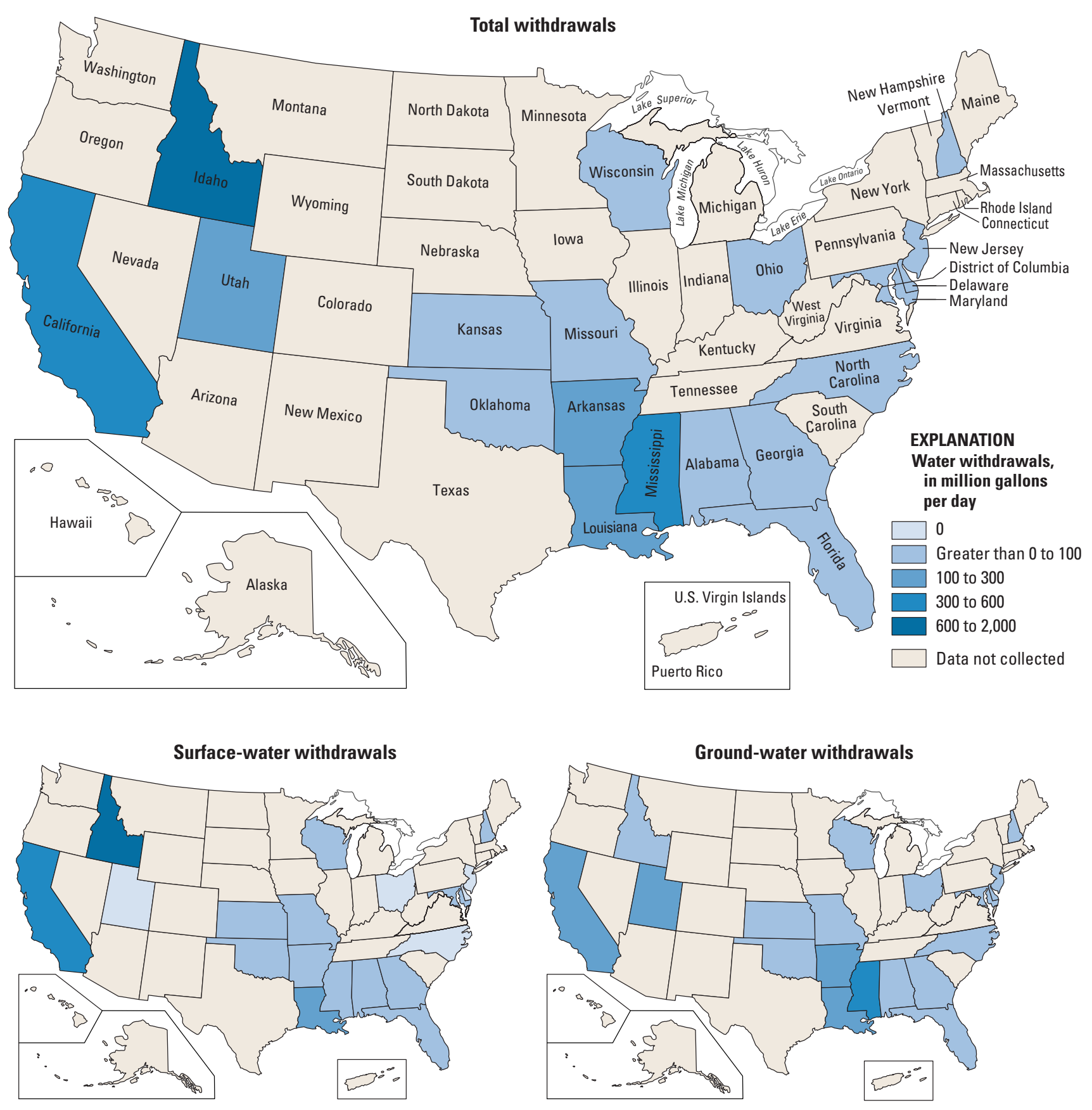

Figure 9. Aquaculture withdrawals by source and State, 2000. 
Industrial water use includes water used for such purposes as fabricating, processing, washing, diluting, cooling, or transporting a product; incorporating water into a product; or for sanitation needs within the manufacturing facility. Some industries that use large amounts of water produce such commodities as food, paper, chemicals, refined petroleum, or primary metals. Water for industrial use may be delivered from a public supplier or be self-supplied. In this report, industrial use refers to self-supplied industrial withdrawals only. Withdrawals were reported as freshwater or saline water. Public-supply deliveries to industrial users and consumptive use were not reported for 2000 .

Industrial withdrawals are listed by State in table 10 . For 2000, withdrawals were an estimated 19,700 Mgal/d, or 22,100 thousand acre-feet per year. Industrial withdrawals were about 5 percent of total withdrawals and about 9 percent of total withdrawals for all categories excluding thermoelectric power. Surface water was the source for 82 percent of total industrial withdrawals. Nearly all (92 percent) of the surface-water withdrawals and nearly all (99 percent) of the ground-water withdrawals for industrial use were freshwater. For 2000, total industrial withdrawals were 11 percent less than during 1995.

The geographic distribution of total, total surface-water, and total ground-water withdrawals for industrial use is shown in figure 10. Louisiana, Indiana, and Texas accounted for almost 38 percent of total industrial withdrawals. The largest fresh surface-water withdrawals were in Louisiana and Indiana, which together accounted for 32 percent of the total fresh surface-water withdrawals. The largest fresh ground-water withdrawals were in Georgia, Louisiana, and Texas, which together accounted for 23 percent of the total fresh ground-water withdrawals. Texas accounted for 71 percent of the saline surface-water withdrawals for industry.

Sources of data for industrial water use included individual facilities and State or Federal permit programs that require reporting of industrial withdrawals or return flows. Industrial withdrawals also were estimated using employment numbers classified by industry group and per employee water-use coefficients.

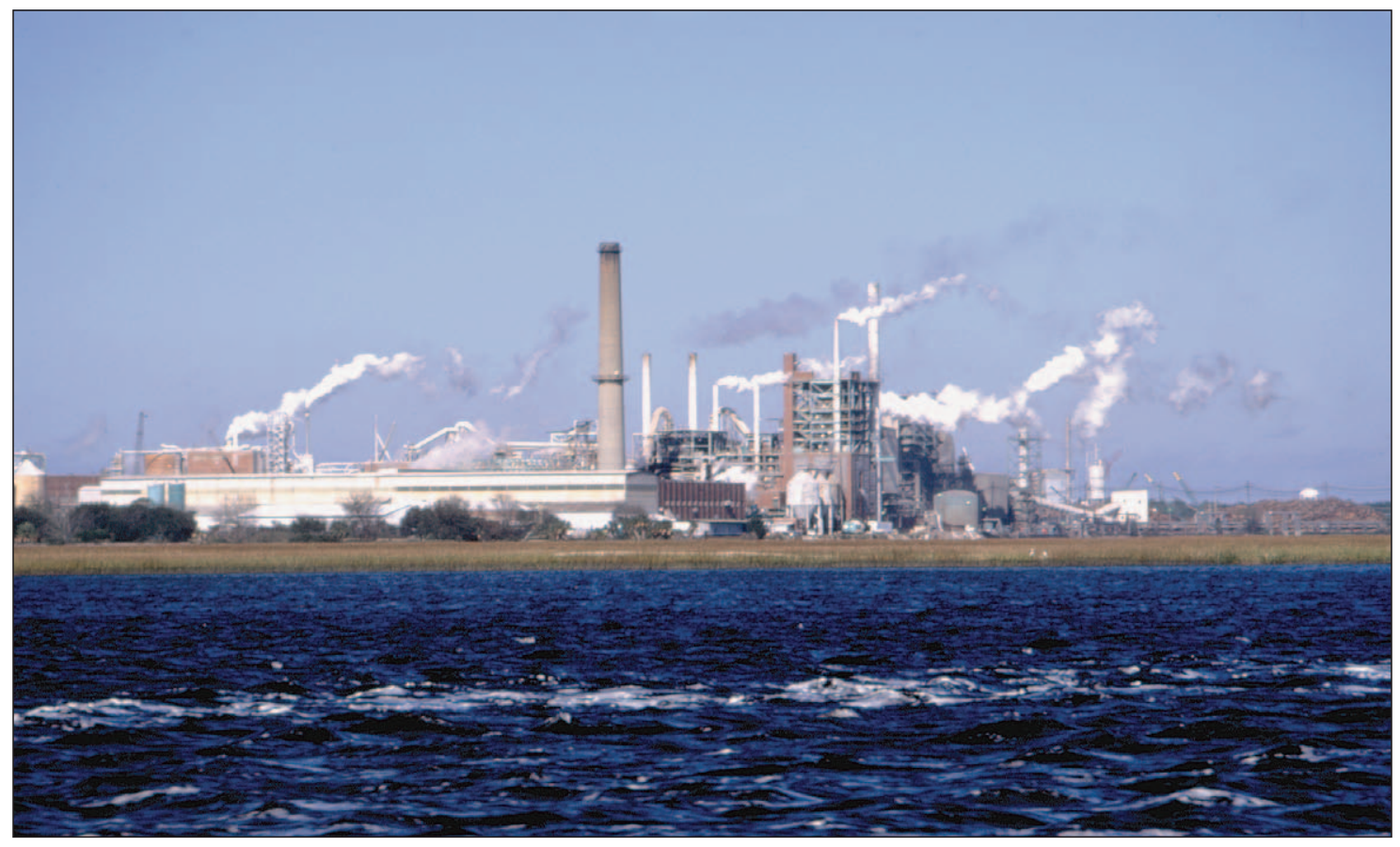

Pulp and paper mill, St. Marys, Georgia (Alan M. Cressler, USGS). 
Table 10. Industrial self-supplied water withdrawals, 2000.

[Figures may not sum to totals because of independent rounding]

\begin{tabular}{|c|c|c|c|c|c|c|c|c|c|c|c|c|}
\hline \multirow{4}{*}{ STATE } & \multicolumn{9}{|c|}{$\begin{array}{c}\text { WITHDRAWALS } \\
\text { (in million gallons per day) }\end{array}$} & \multirow{2}{*}{\multicolumn{3}{|c|}{$\begin{array}{c}\text { WITHDRAWALS } \\
\text { (in thousand acre-feet } \\
\text { per year) }\end{array}$}} \\
\hline & \multicolumn{6}{|c|}{ By source and type } & & & & & & \\
\hline & \multicolumn{3}{|c|}{ Ground water } & \multicolumn{3}{|c|}{ Surface water } & \multicolumn{3}{|c|}{ Total } & \multicolumn{2}{|c|}{ By type } & \multirow[b]{2}{*}{ Total } \\
\hline & Fresh & Saline & Total & Fresh & Saline & Total & Fresh & Saline & Total & Fresh & Saline & \\
\hline Alabama ..................... & 56.0 & 0 & 56.0 & 777 & 0 & 777 & 833 & 0 & 833 & 934 & 0 & 934 \\
\hline Alaska ........................ & 4.32 & 0 & 4.32 & 3.80 & 3.86 & 7.66 & 8.12 & 3.86 & 12.0 & 9.10 & 4.33 & 13.4 \\
\hline Arizona ...................... & 19.8 & 0 & 19.8 & 0 & 0 & 0 & 19.8 & 0 & 19.8 & 22.2 & 0 & 22.2 \\
\hline Arkansas ................. & 67.0 & .08 & 67.1 & 66.8 & 0 & 66.8 & 134 & .08 & 134 & 150 & .09 & 150 \\
\hline California ................ & 183 & 0 & 183 & 5.65 & 13.6 & 19.3 & 188 & 13.6 & 202 & 211 & 15.3 & 226 \\
\hline Colorado ................... & 23.6 & 0 & 23.6 & 96.4 & 0 & 96.4 & 120 & 0 & 120 & 135 & 0 & 135 \\
\hline Connecticut ............. & 4.13 & 0 & 4.13 & 6.61 & 0 & 6.61 & 10.7 & 0 & 10.7 & 12.0 & 0 & 12.0 \\
\hline Delaware ................... & 17.0 & 0 & 17.0 & 42.5 & 3.25 & 45.7 & 59.4 & 3.25 & 62.7 & 66.6 & 3.64 & 70.3 \\
\hline District of Columbia & 0 & 0 & 0 & 0 & 0 & 0 & 0 & 0 & 0 & 0 & 0 & 0 \\
\hline Florida ……................... & 216 & 0 & 216 & 74.7 & 1.18 & 75.9 & 291 & 1.18 & 292 & 326 & 1.32 & 328 \\
\hline 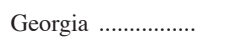 & 290 & 0 & 290 & 333 & 30.0 & 363 & 622 & 30.0 & 652 & 698 & 33.6 & 731 \\
\hline Hawaii ......................... & 14.5 & .85 & 15.4 & 0 & 0 & 0 & 14.5 & .85 & 15.4 & 16.2 & .95 & 17.2 \\
\hline Idaho ........................... & 35.8 & 0 & 35.8 & 19.7 & 0 & 19.7 & 55.5 & 0 & 55.5 & 62.2 & 0 & 62.2 \\
\hline Illinois ........................ & 132 & 0 & 132 & 259 & 0 & 259 & 391 & 0 & 391 & 438 & 0 & 438 \\
\hline Indiana ......................... & 99.7 & 0 & 99.7 & 2,300 & 0 & 2,300 & 2,400 & 0 & 2,400 & 2,690 & 0 & 2,690 \\
\hline 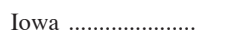 & 226 & 0 & 226 & 11.7 & 0 & 11.7 & 237 & 0 & 237 & 266 & 0 & 266 \\
\hline Kansas ......................... & 46.6 & 0 & 46.6 & 6.74 & 0 & 6.74 & 53.3 & 0 & 53.3 & 59.8 & 0 & 59.8 \\
\hline Kentucky ................... & 95.2 & 0 & 95.2 & 222 & 0 & 222 & 317 & 0 & 317 & 356 & 0 & 356 \\
\hline Louisiana .................. & 285 & 0 & 285 & 2,400 & 0 & 2,400 & 2,680 & 0 & 2,680 & 3,010 & 0 & 3,010 \\
\hline Maine ……….................. & 9.90 & 0 & 9.90 & 237 & 0 & 237 & 247 & 0 & 247 & 277 & 0 & 277 \\
\hline Maryland .................. & 15.9 & 0 & 15.9 & 49.9 & 227 & 277 & 65.8 & 227 & 292 & 73.8 & 254 & 328 \\
\hline Massachusetts ....... & 10.7 & 0 & 10.7 & 26.2 & 0 & 26.2 & 36.8 & 0 & 36.8 & 41.3 & 0 & 41.3 \\
\hline Michigan ...................... & 110 & 0 & 110 & 589 & 0 & 589 & 698 & 0 & 698 & 782 & 0 & 782 \\
\hline Minnesota .............. & 56.3 & 0 & 56.3 & 97.8 & 0 & 97.8 & 154 & 0 & 154 & 173 & 0 & 173 \\
\hline Mississippi ............. & 118 & 0 & 118 & 124 & 0 & 124 & 242 & 0 & 242 & 271 & 0 & 271 \\
\hline Missouri .................... & 29.2 & 0 & 29.2 & 33.5 & 0 & 33.5 & 62.7 & 0 & 62.7 & 70.3 & 0 & 70.3 \\
\hline Montana ..................... & 31.9 & 0 & 31.9 & 29.3 & 0 & 29.3 & 61.3 & 0 & 61.3 & 68.7 & 0 & 68.7 \\
\hline Nebraska ..................... & 35.5 & 0 & 35.5 & 2.60 & 0 & 2.60 & 38.1 & 0 & 38.1 & 42.7 & 0 & 42.7 \\
\hline Nevada .......................... & 5.29 & 0 & 5.29 & 5.00 & 0 & 5.00 & 10.3 & 0 & 10.3 & 11.5 & 0 & 11.5 \\
\hline New Hampshire ... & 6.95 & 0 & 6.95 & 37.9 & 0 & 37.9 & 44.9 & 0 & 44.9 & 50.3 & 0 & 50.3 \\
\hline New Jersey ............ & 65.3 & 0 & 65.3 & 66.2 & 0 & 66.2 & 132 & 0 & 132 & 147 & 0 & 147 \\
\hline New Mexico ......... & 8.80 & 0 & 8.80 & 1.67 & 0 & 1.67 & 10.5 & 0 & 10.5 & 11.7 & 0 & 11.7 \\
\hline New York ................... & 145 & 0 & 145 & 152 & 0 & 152 & 297 & 0 & 297 & 333 & 0 & 333 \\
\hline North Carolina ...... & 25.6 & 0 & 25.6 & 267 & 0 & 267 & 293 & 0 & 293 & 329 & 0 & 329 \\
\hline North Dakota ......... & 6.88 & 0 & 6.88 & 10.7 & 0 & 10.7 & 17.6 & 0 & 17.6 & 19.7 & 0 & 19.7 \\
\hline 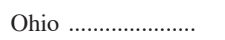 & 162 & 0 & 162 & 645 & 0 & 645 & 807 & 0 & 807 & 905 & 0 & 905 \\
\hline Oklahoma .................. & 6.83 & 0 & 6.83 & 19.1 & 0 & 19.1 & 25.9 & 0 & 25.9 & 29.1 & 0 & 29.1 \\
\hline Oregon ………............. & 12.1 & 0 & 12.1 & 183 & 0 & 183 & 195 & 0 & 195 & 218 & 0 & 218 \\
\hline Pennsylvania .......... & 155 & 0 & 155 & 1,030 & 0 & 1,030 & 1,190 & 0 & 1,190 & 1,330 & 0 & 1,330 \\
\hline Rhode Island ......... & 2.19 & 0 & 2.19 & 2.09 & 0 & 2.09 & 4.28 & 0 & 4.28 & 4.80 & 0 & 4.80 \\
\hline South Carolina ...... & 50.9 & 0 & 50.9 & 514 & 0 & 514 & 565 & 0 & 565 & 633 & 0 & 633 \\
\hline South Dakota ......... & 3.16 & 0 & 3.16 & 1.96 & 0 & 1.96 & 5.12 & 0 & 5.12 & 5.74 & 0 & 5.74 \\
\hline Tennessee ................ & 56.3 & 0 & 56.3 & 785 & 0 & 785 & 842 & 0 & 842 & 944 & 0 & 944 \\
\hline Texas ....………........... & 244 & .50 & 244 & 1,200 & 906 & 2,110 & 1,450 & 907 & 2,350 & 1,620 & 1,020 & 2,640 \\
\hline Utah ......................... & 34.3 & 5.08 & 39.4 & 8.38 & 0 & 8.38 & 42.7 & 5.08 & 47.8 & 47.8 & 5.69 & 53.5 \\
\hline Vermont .................... & 2.05 & 0 & 2.05 & 4.86 & 0 & 4.86 & 6.91 & 0 & 6.91 & 7.75 & 0 & 7.75 \\
\hline Virginia .................... & 104 & 0 & 104 & 365 & 53.3 & 419 & 470 & 53.3 & 523 & 526 & 59.7 & 586 \\
\hline Washington ............. & 138 & 0 & 138 & 439 & 39.9 & 479 & 577 & 39.9 & 617 & 647 & 44.7 & 692 \\
\hline West Virginia ........ & 9.70 & 0 & 9.70 & 958 & 0 & 958 & 968 & 0 & 968 & 1,090 & 0 & 1,090 \\
\hline Wisconsin ................ & 83.0 & 0 & 83.0 & 364 & 0 & 364 & 447 & 0 & 447 & 501 & 0 & 501 \\
\hline Wyoming ................... & 4.31 & 0 & 4.31 & 1.47 & 0 & 1.47 & 5.78 & 0 & 5.78 & 6.48 & 0 & 6.48 \\
\hline Puerto Rico ............. & 11.2 & 0 & 11.2 & 0 & 0 & 0 & 11.2 & 0 & 11.2 & 12.5 & 0 & 12.5 \\
\hline U.S. Virgin Islands & .22 & 0 & .22 & 3.12 & 0 & 3.12 & 3.34 & 0 & 3.34 & 3.74 & 0 & 3.74 \\
\hline TOTAL & 3,570 & 6.51 & 3,580 & 14,900 & 1,280 & 16,200 & 18,500 & 1,280 & 19,700 & 20,700 & 1,440 & 22,100 \\
\hline
\end{tabular}



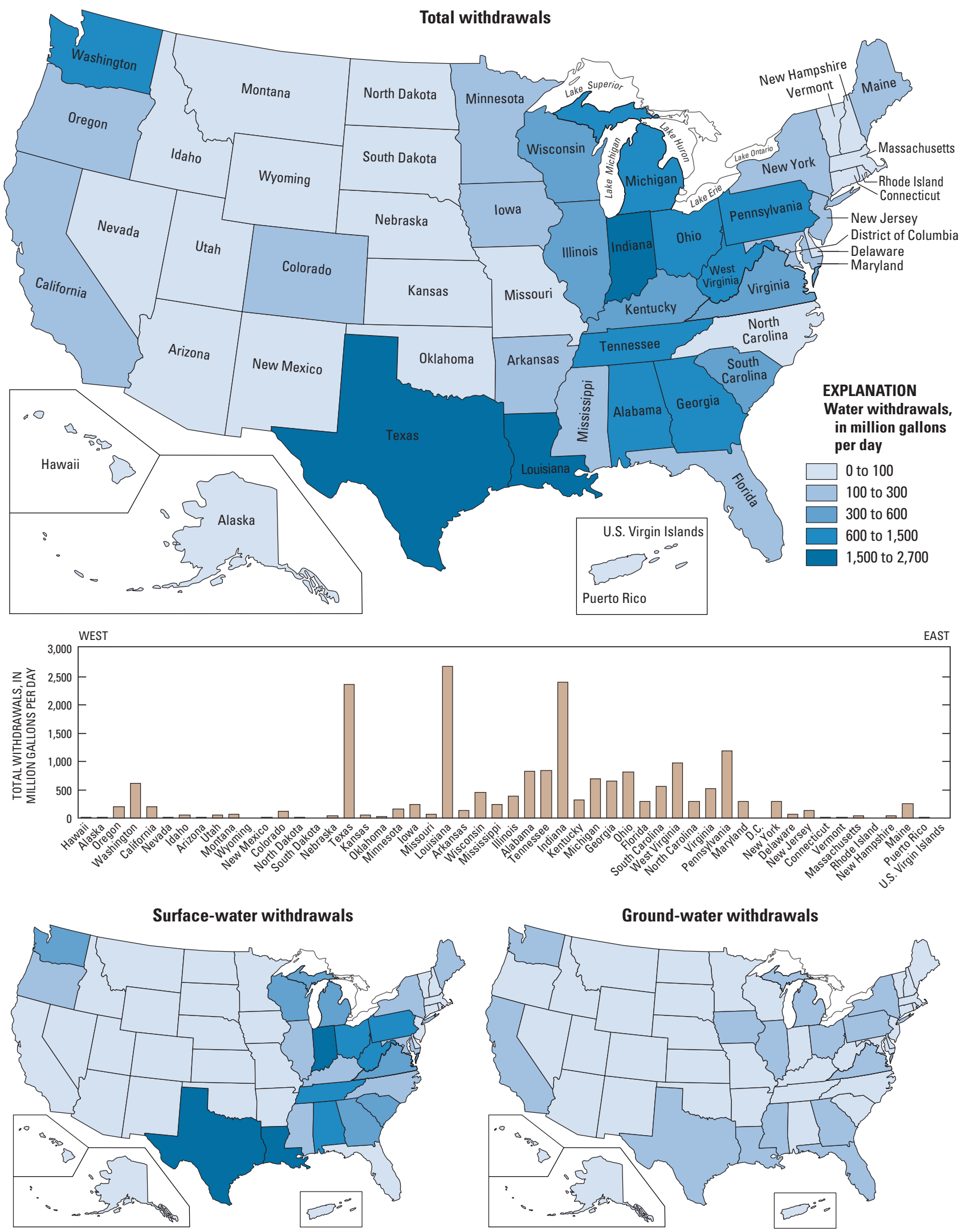

Figure 10. Industrial withdrawals by source and State, 2000. 
Mining water use is water for the extraction of minerals that may be in the form of such solids as coal, iron, sand, and gravel; such liquids as crude petroleum; and such gases as natural gas. The category includes quarrying, milling (crushing, screening, washing, and flotation of mined materials), re-injecting extracted water for secondary oil recovery, and other operations associated with mining activities. All mining withdrawals were considered selfsupplied. Water withdrawals were reported as freshwater or saline water. Dewatering was not reported as a mining withdrawal unless the water was used beneficially, such as dampening roads for dust control. For 2000, consumptive use was not reported for mining.

For 2000, the estimate of mining water use for the United States was based on estimates of total withdrawals for mining in 22 States, rather than on estimates from all States. These 22 States included the 12 States with the largest mining withdrawals during 1995, and 10 other States in which mining water-use data had been collected as part of a broader State water-use program for 2000. The 22 States that reported for 2000 accounted for 83 percent of the total mining water withdrawals during 1995. During 1995, withdrawals for mining accounted for a small percentage of the total water use, less than 1 percent of the total water withdrawals for all categories.

Mining withdrawals for 2000 are listed by State in table 11. For 2000, an estimated 3,490 Mgal/d, or 3,920 thousand acre-feet per year, were used. Mining withdrawals were nearly 1 percent of total withdrawals and less than 2 percent of total withdrawals for all categories excluding thermoelectric power. Ground water was the source for 58 percent of total withdrawals for mining. Most of the ground-water withdrawals for mining (62 percent) were saline, and most of the surface-water withdrawals ( 85 percent) were freshwater. Saline groundwater withdrawals and fresh surface-water withdrawals each represented 36 percent of the total withdrawals for mining.

Total withdrawals for mining for the 22 States that reported data during both 1995 and 2000 increased about 11 percent during this time period; ground-water withdrawals increased about 15 percent; and surface-water withdrawals increased 7 percent. Saline-water withdrawals increased 32 percent. Freshwater withdrawals remained about the same for 2000 .

The geographic distribution of total, total freshwater, and total saline-water withdrawals is shown in figure 11. Texas, Minnesota, and Wyoming accounted for 46 percent of the total withdrawals for mining. Iron ore mining in Minnesota, sand and gravel operations in Nebraska, and sand operations in New Jersey accounted for the largest fresh surface-water withdrawals. Gas and oil operations in Alaska, California, Oklahoma, Texas, and Wyoming were responsible for the large saline ground-water withdrawals in those States. Mineral salt extraction from the Great Salt Lake in Utah accounted for the largest saline surface-water withdrawals in the United States.

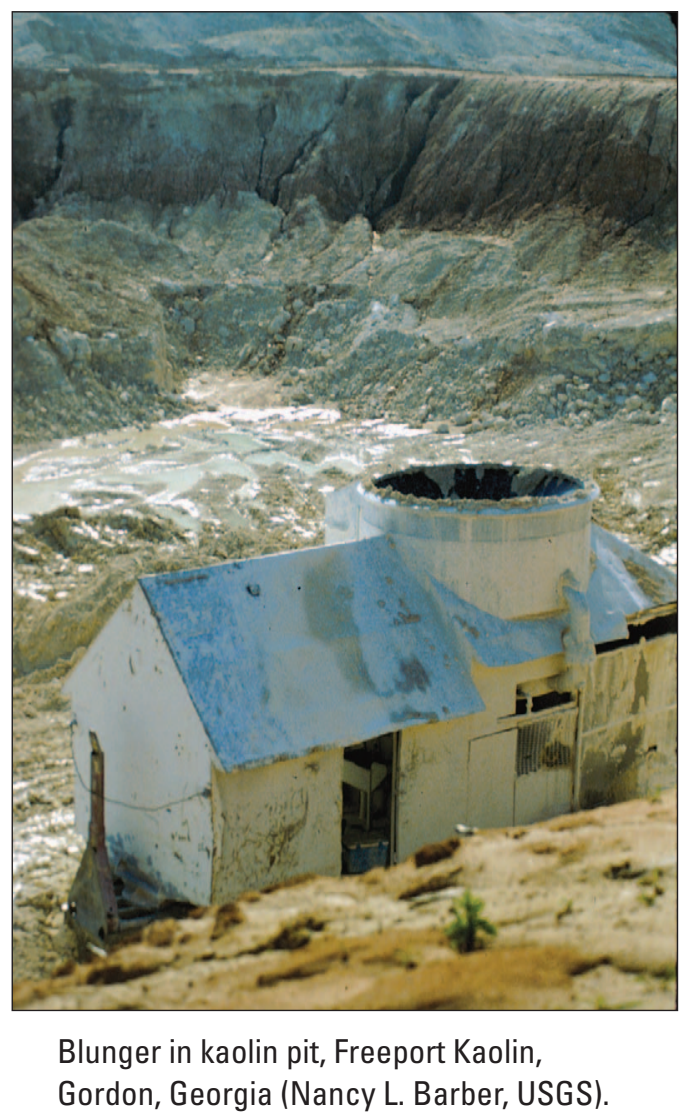

Sources of data for water use for mining included surveys of mining operations or State and Federal agencies that collect water withdrawal or discharge data for mining sites or mineral tonnages and associated coefficients. A determination of water withdrawals that are put to beneficial use in mining operations can be difficult to determine, especially when dewatering is necessary for extraction of the mineral. Water produced from dewatering varies in quality from fresh to saline, and generally is disposed of through surface discharge, ponding, or re-injection. Some of the less-mineralized water may be re-used for irrigation or livestock. 
Table 11. Mining water withdrawals, 2000.

[Figures may not sum to totals because of independent rounding. - -, data not collected]

\begin{tabular}{|c|c|c|c|c|c|c|c|c|c|c|c|c|}
\hline \multirow{4}{*}{ STATE } & \multicolumn{9}{|c|}{$\begin{array}{c}\text { WITHDRAWALS } \\
\text { (in million gallons per day) }\end{array}$} & \multicolumn{3}{|c|}{$\begin{array}{l}\text { WITHDRAWALS } \\
\text { (in thousand acre-feet per year) }\end{array}$} \\
\hline & \multicolumn{6}{|c|}{ By source and type } & & & & & & \\
\hline & \multicolumn{3}{|c|}{ Ground water } & \multicolumn{3}{|c|}{ Surface water } & \multicolumn{3}{|c|}{ Total } & \multicolumn{2}{|c|}{ By type } & \multirow[b]{2}{*}{ Total } \\
\hline & Fresh & Saline & Total & Fresh & Saline & Total & Fresh & Saline & Total & Fresh & Saline & \\
\hline Alabama ....................... & - & - & - & - & - & - & - & - & - & - & - & - \\
\hline Alaska ........................ & 0.01 & 90.4 & 90.4 & 27.4 & 49.5 & 76.9 & 27.4 & 140 & 167 & 30.7 & 157 & 188 \\
\hline Arizona ………........... & 81.2 & 8.17 & 89.4 & 4.43 & 0 & 4.43 & 85.7 & 8.17 & 93.8 & 96.0 & 9.16 & 105 \\
\hline Arkansas .................... & .21 & 0 & .21 & 2.57 & 0 & 2.57 & 2.78 & 0 & 2.78 & 3.12 & 0 & 3.12 \\
\hline 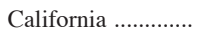 & 21.0 & 152 & 173 & 2.71 & .46 & 3.17 & 23.7 & 153 & 177 & 26.6 & 171 & 198 \\
\hline Colorado ..................... & - & - & - & - & - & - & - & - & - & - & - & - \\
\hline Connecticut ........... & - & - & - & - & - & - & - & - & - & - & - & - \\
\hline Delaware ................. & - & - & - & - & - & - & - & - & - & - & - & - \\
\hline District of Columbia & - & - & - & - & - & - & - & - & - & - & - & - \\
\hline Florida ……….............. & 160 & 0 & 160 & 57.8 & 0 & 57.8 & 217 & 0 & 217 & 244 & 0 & 244 \\
\hline Georgia ...................... & 7.75 & 0 & 7.75 & 2.05 & 0 & 2.05 & 9.80 & 0 & 9.80 & 11.0 & 0 & 11.0 \\
\hline Hawaii ....................... & - & - & - & - & - & - & - & - & - & - & - & - \\
\hline Idaho ....................... & - & - & - & - & - & - & - & - & - & - & - & - \\
\hline Illinois ........................ & - & - & - & - & - & - & - & - & - & - & - & - \\
\hline Indiana .......................... & 4.20 & 0 & 4.20 & 78.3 & 0 & 78.3 & 82.5 & 0 & 82.5 & 92.5 & 0 & 92.5 \\
\hline Iowa ........................... & 2.49 & 0 & 2.49 & 30.3 & 0 & 30.3 & 32.8 & 0 & 32.8 & 36.8 & 0 & 36.8 \\
\hline Kansas ....................... & 14.0 & 0 & 14.0 & 17.4 & 0 & 17.4 & 31.4 & 0 & 31.4 & 35.2 & 0 & 35.2 \\
\hline Kentucky ............... & - & - & - & - & - & - & - & - & - & - & - & - \\
\hline Louisiana ................ & - & - & - & - & - & - & - & - & - & - & - & - \\
\hline Maine …….................... & - & - & - & - & - & - & - & - & - & - & - & - \\
\hline Maryland ................... & 4.21 & 0 & 4.21 & 4.10 & .02 & 4.12 & 8.31 & .02 & 8.33 & 9.32 & .02 & 9.34 \\
\hline Massachusetts ...... & - & - & - & - & - & - & - & - & - & - & - & - \\
\hline 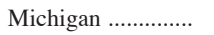 & - & - & - & - & - & - & - & - & - & - & - & - \\
\hline Minnesota .............. & 6.90 & 0 & 6.90 & 581 & 0 & 581 & 588 & 0 & 588 & 659 & 0 & 659 \\
\hline Mississippi ............ & - & - & - & - & - & - & - & - & - & - & - & - \\
\hline Missouri .................... & 4.10 & 0 & 4.10 & 12.8 & 0 & 12.8 & 16.9 & 0 & 16.9 & 19.0 & 0 & 19.0 \\
\hline Montana ..................... & - & - & - & - & - & - & - & - & - & - & - & - \\
\hline Nebraska .................... & 5.64 & 4.55 & 10.2 & 122 & 0 & 122 & 128 & 4.55 & 132 & 143 & 5.10 & 148 \\
\hline Nevada ...................... & - & - & - & - & - & - & - & - & - & - & - & - \\
\hline New Hampshire ... & .08 & 0 & .08 & 6.72 & 0 & 6.72 & 6.80 & 0 & 6.80 & 7.62 & 0 & 7.62 \\
\hline New Jersey ........... & 6.12 & 0 & 6.12 & 104 & 0 & 104 & 110 & 0 & 110 & 124 & 0 & 124 \\
\hline New Mexico ......... & - & - & - & - & - & - & - & - & - & - & - & - \\
\hline New York ................... & - & - & - & - & - & - & - & - & - & - & - & - \\
\hline North Carolina ...... & 36.4 & 0 & 36.4 & 0 & 0 & 0 & 36.4 & 0 & 36.4 & 40.8 & 0 & 40.8 \\
\hline North Dakota ......... & - & - & - & - & - & - & - & - & - & - & - & - \\
\hline Ohio ……................ & 53.1 & 0 & 53.1 & 35.5 & 0 & 35.5 & 88.5 & 0 & 88.5 & 99.2 & 0 & 99.2 \\
\hline Oklahoma .............. & 2.25 & 256 & 258 & .23 & 0 & .23 & 2.48 & 256 & 258 & 2.78 & 287 & 290 \\
\hline Oregon …….............. & - & - & - & - & - & - & - & - & - & - & - & - \\
\hline Pennsylvania ......... & 162 & 0 & 162 & 20.9 & 0 & 20.9 & 182 & 0 & 182 & 205 & 0 & 205 \\
\hline Rhode Island ........ & - & - & - & - & - & - & - & - & - & - & - & - \\
\hline South Carolina ...... & - & - & - & - & - & - & - & - & - & - & - & - \\
\hline South Dakota ........ & - & - & - & - & - & - & - & - & - & - & - & - \\
\hline Tennessee ................ & - & - & - & - & - & - & - & - & - & - & - & - \\
\hline Texas ........................ & 129 & 504 & 633 & 91.5 & 0 & 91.5 & 220 & 504 & 724 & 247 & 565 & 812 \\
\hline Utah ............................... & 8.60 & 21.5 & 30.1 & 17.7 & 177 & 194 & 26.3 & 198 & 225 & 29.4 & 222 & 252 \\
\hline Vermont .................... & - & - & - & - & - & - & - & - & - & - & - & - \\
\hline 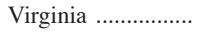 & - & - & - & - & - & - & - & - & - & - & - & - \\
\hline Washington ............ & - & - & - & - & - & - & - & - & - & - & - & - \\
\hline West Virginia ........ & - & - & - & - & - & - & - & - & - & - & - & - \\
\hline Wisconsin ................... & - & - & - & - & - & - & - & - & - & - & - & - \\
\hline 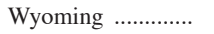 & 58.8 & 222 & 280 & 20.7 & 0 & 20.7 & 79.5 & 222 & 301 & 89.1 & 248 & 338 \\
\hline Puerto Rico ............ & - & - & - & - & - & - & - & - & - & - & - & - \\
\hline U.S. Virgin Islands & - & - & - & - & - & - & - & - & - & - & - & - \\
\hline TOTAL & 767 & 1,260 & 2,030 & 1,240 & 227 & 1,470 & 2,010 & 1,490 & 3,490 & 2,250 & 1,660 & 3,920 \\
\hline
\end{tabular}



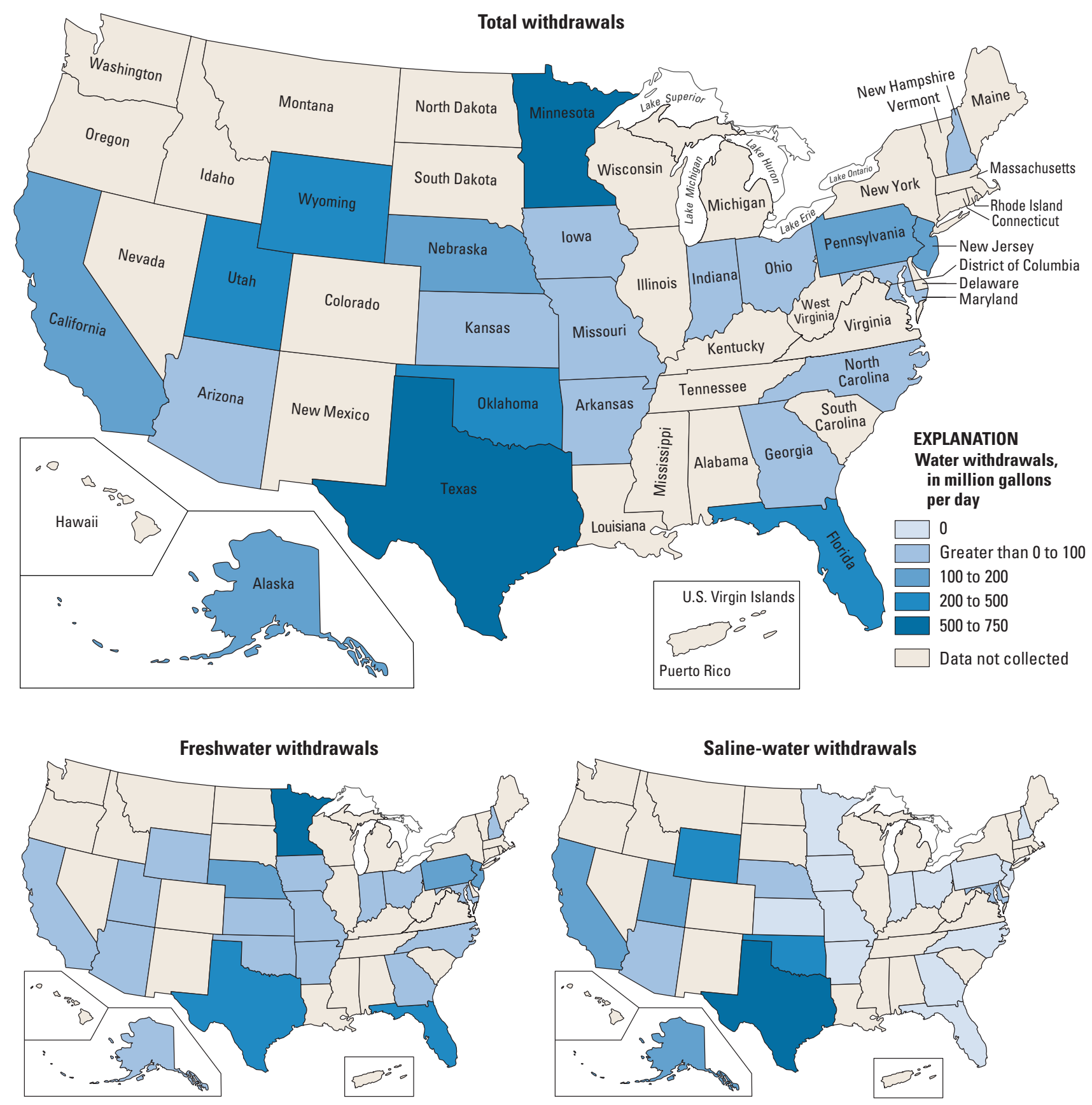

Figure 11. Mining withdrawals by water quality and State, 2000. 
Water for thermoelectric power is used in generating electricity with steam-driven turbine generators. For 2000, thermoelectric-power water withdrawals were compiled by cooling-system type because cooling-system type is the primary determinant for the amount of consumptive use relative to withdrawals. Once-through cooling refers to cooling systems in which water is withdrawn from a source, circulated through the heat exchangers, and then returned to a surface-water body. Closed-loop cooling refers to cooling systems in which water is withdrawn from a source, circulated through heat exchangers, cooled, and then recycled. Subsequent water withdrawals for a closed-loop system are used to replace water lost to evaporation, blowdown, drift, and leakage. Closed-loop cooling results in larger consumptive-use values relative to withdrawals. Water withdrawals were reported as freshwater or saline water. For 2000, public-supply deliveries to thermoelectric-power plants, water use by fuel type, consumptive use, and the amount of power generated were not reported.

Thermoelectric-power withdrawals are listed by State in table 12. The total quantity of water withdrawn for thermoelectric power for 2000 was an estimated 195,000 Mgal/d, or 219,000 thousand acre-feet per year. Surface water was the source for more than 99 percent of total thermoelectricpower withdrawals. Nearly one-third of the surface water was saline. Saline withdrawals from surface water sources accounted for 96 percent of the total saline withdrawals for all categories. Thermoelectric-power withdrawals accounted for 48 percent of total water use, 39 percent of total freshwater withdrawals for all categories, and 52 percent of fresh surface-water withdrawals. Estimates of freshwater and saline-water withdrawals were each about 3 percent more for 2000 than for 1995.

The geographic distribution of total, total freshwater, and total saline-water withdrawals for thermoelectric power is shown in figure 12. The largest total water withdrawals were in Texas, where both freshwater and saline water were utilized for cooling purposes. Illinois, Texas, and Tennessee combined accounted for 22 percent of total freshwater withdrawals. California and Florida accounted for 41 percent of saline surface-water withdrawals. Freshwater and salinewater withdrawals in the eastern States were 83 percent of total water withdrawals. This pattern of withdrawals exists partly because the power-production infrastructure was established in the eastern States to take advantage of the plentiful water supply in the Great Lakes, along major rivers, and along the coast in order to meet electricity demand from large urban and industrial centers.

In contrast, the Pacific Northwest utilizes hydroelectricpower generation to supply a substantial part of the regional demand for electricity. For example, in Idaho, power is supplied primarily by hydroelectric-power generation, and, therefore, Idaho reported no withdrawals for thermoelectric power. Hydroelectric power, an instream use, is not included in this report.

Thermoelectric-power withdrawals are listed by coolingsystem type and by State in table 13. Power plants equipped with once-through cooling systems accounted for 91 percent of water withdrawals for thermoelectric power. Plants equipped with closed-loop cooling systems withdrew the remaining 9 percent of the water. Cooling technologies that require less water also allow for the production of thermoelectric power in areas where water is scarce or strictly managed (Michelletti and Burns, 2002). Such water-scarce States as Arizona, Nevada, and New Mexico utilized closedloop cooling systems rather than the more water-intensive once-through cooling systems during 2000. During 2000, about 75 percent of the generating units using closed-loop cooling systems reported rates of consumptive use greater than 50 percent (U.S. Department of Energy, Energy Information Administration, 2003a, 2003b).

In coastal areas, the use of saline water instead of freshwater expands the overall available water supply. More than 90 percent of the thermoelectric-power withdrawals were saline in Puerto Rico (100 percent), U.S. Virgin Islands (100 percent), Rhode Island, California, Massachusetts, Connecticut, Florida, and Maryland. Saline ground-water use by geothermal power plants in Nevada (78.7 Mgal/d), California (32.9 Mgal/d), and Utah (0.87 Mgal/d) was compiled, but excluded from the total water withdrawals and tables in this report. Thermoelectric-power withdrawals for Hawaii were all ground water for once-through cooling systems (1,200 Mgal/d). Saline ground-water withdrawals supplied 96 percent $(1,150 \mathrm{Mgal} / \mathrm{d})$ of the total. Hawaii's thermoelectric-power data were excluded from the total water withdrawals and tables in this report.

Sources of data for thermoelectric-power water use included individual facilities, State permitting or regulatory agencies, or the USDOE-EIA. Generally, relatively complete files on water withdrawals and power generation were maintained by these entities for 2000 . 
Table 12. Thermoelectric-power water withdrawals, 2000.

[Figures may not sum to totals because of independent rounding]

\begin{tabular}{|c|c|c|c|c|c|c|c|c|c|c|}
\hline \multirow{4}{*}{ STATE } & \multicolumn{7}{|c|}{$\begin{array}{c}\text { WITHDRAWALS } \\
\text { (in million gallons per day) }\end{array}$} & \multicolumn{3}{|c|}{$\begin{array}{c}\text { WITHDRAWALS } \\
\text { (in thousand acre-feet per year) }\end{array}$} \\
\hline & \multicolumn{4}{|c|}{ By source and type } & \multirow{2}{*}{\multicolumn{3}{|c|}{ Total }} & & & \\
\hline & \multirow{2}{*}{$\begin{array}{l}\text { Ground water } \\
\text { Fresh }\end{array}$} & \multicolumn{3}{|c|}{ Surface water } & & & & \multicolumn{2}{|c|}{ By type } & \multirow[b]{2}{*}{ Total } \\
\hline & & Fresh & Saline & Total & Fresh & Saline & Total & Fresh & Saline & \\
\hline Alabama ................................ & 0 & 8,190 & 0 & 8,190 & 8,190 & 0 & 8,190 & 9,180 & 0 & 9,180 \\
\hline Alaska ............................. & 4.65 & 28.9 & 0 & 28.9 & 33.6 & 0 & 33.6 & 37.6 & 0 & 37.6 \\
\hline Arizona ………….............. & 74.3 & 26.2 & 0 & 26.2 & 100 & 0 & 100 & 113 & 0 & 113 \\
\hline Arkansas .......................... & 2.92 & 2,170 & 0 & 2,170 & 2,180 & 0 & 2,180 & 2,440 & 0 & 2,440 \\
\hline California …..................... & 3.23 & 349 & 12,600 & 12,900 & 352 & 12,600 & 12,900 & 395 & 14,100 & 14,500 \\
\hline Colorado .......................... & 16.1 & 122 & 0 & 122 & 138 & 0 & 138 & 155 & 0 & 155 \\
\hline Connecticut ..................... & .08 & 186 & 3,440 & 3,630 & 187 & 3,440 & 3,630 & 209 & 3,860 & 4,070 \\
\hline Delaware .......................... & .47 & 366 & 738 & 1,100 & 366 & 738 & 1,100 & 411 & 827 & 1,240 \\
\hline District of Columbia .. & 0 & 9.69 & 0 & 9.69 & 9.69 & 0 & 9.69 & 10.9 & 0 & 10.9 \\
\hline Florida ………….............. & 29.5 & 629 & 12,000 & 12,600 & 658 & 12,000 & 12,600 & 738 & 13,400 & 14,100 \\
\hline Georgia ........................... & 1.03 & 3,240 & 61.7 & 3,310 & 3,250 & 61.7 & 3,310 & 3,640 & 69.2 & 3,710 \\
\hline Hawaii ............................. & 0 & 0 & 0 & 0 & 0 & 0 & 0 & 0 & 0 & 0 \\
\hline Idaho …............................ & 0 & 0 & 0 & 0 & 0 & 0 & 0 & 0 & 0 & 0 \\
\hline 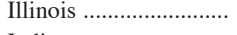 & 5.75 & 11,300 & 0 & 11,300 & 11,300 & 0 & 11,300 & 12,600 & 0 & 12,600 \\
\hline Indiana ................................ & 2.58 & 6,700 & 0 & 6,700 & 6,700 & 0 & 6,700 & 7,510 & 0 & 7,510 \\
\hline Iowa ………..................... & 11.9 & 2,530 & 0 & 2,530 & 2,540 & 0 & 2,540 & 2,850 & 0 & 2,850 \\
\hline Kansas ............................. & 14.9 & 2,240 & 0 & 2,240 & 2,260 & 0 & 2,260 & 2,530 & 0 & 2,530 \\
\hline Kentucky ……................. & 2.71 & 3,250 & 0 & 3,250 & 3,260 & 0 & 3,260 & 3,650 & 0 & 3,650 \\
\hline Louisiana ........................ & 28.4 & 5,580 & 0 & 5,580 & 5,610 & 0 & 5,610 & 6,290 & 0 & 6,290 \\
\hline Maine ……………........... & 4.92 & 108 & 295 & 403 & 113 & 295 & 408 & 127 & 330 & 457 \\
\hline Maryland ………….......... & 1.80 & 377 & 6,260 & 6,640 & 379 & 6,260 & 6,640 & 425 & 7,020 & 7,440 \\
\hline Massachusetts ................ & 0 & 108 & 3,610 & 3,720 & 108 & 3,610 & 3,720 & 121 & 4,050 & 4,170 \\
\hline Michigan ............................ & 0 & 7,710 & 0 & 7,710 & 7,710 & 0 & 7,710 & 8,640 & 0 & 8,640 \\
\hline Minnesota ........................ & 4.17 & 2,260 & 0 & 2,260 & 2,270 & 0 & 2,270 & 2,540 & 0 & 2,540 \\
\hline Mississippi .................... & 43.5 & 318 & 148 & 467 & 362 & 148 & 510 & 406 & 166 & 572 \\
\hline Missouri .......................... & 12.2 & 5,620 & 0 & 5,620 & 5,640 & 0 & 5,640 & 6,320 & 0 & 6,320 \\
\hline Montana ................................ & 0 & 110 & 0 & 110 & 110 & 0 & 110 & 123 & 0 & 123 \\
\hline Nebraska ............................ & 6.87 & 2,810 & 0 & 2,810 & 2,820 & 0 & 2,820 & 3,160 & 0 & 3,160 \\
\hline Nevada ................................ & 12.0 & 24.7 & 0 & 24.7 & 36.7 & 0 & 36.7 & 41.1 & 0 & 41.1 \\
\hline New Hampshire .......... & .71 & 235 & 761 & 997 & 236 & 761 & 997 & 265 & 854 & 1,120 \\
\hline New Jersey ..................... & 2.24 & 648 & 3,390 & 4,040 & 650 & 3,390 & 4,040 & 729 & 3,800 & 4,530 \\
\hline New Mexico ................. & 11.4 & 45.0 & 0 & 45.0 & 56.4 & 0 & 56.4 & 63.2 & 0 & 63.2 \\
\hline New York .......................... & 0 & 4,040 & 5,010 & 9,050 & 4,040 & 5,010 & 9,050 & 4,530 & 5,610 & 10,100 \\
\hline North Carolina ................ & .09 & 7,850 & 1,620 & 9,470 & 7,850 & 1,620 & 9,470 & 8,800 & 1,810 & 10,600 \\
\hline North Dakota ................... & 0 & 902 & 0 & 902 & 902 & 0 & 902 & 1,010 & 0 & 1,010 \\
\hline Ohio ……………............ & 7.57 & 8,590 & 0 & 8,590 & 8,590 & 0 & 8,590 & 9,630 & 0 & 9,630 \\
\hline Oklahoma ……................ & 3.27 & 143 & 0 & 143 & 146 & 0 & 146 & 164 & 0 & 164 \\
\hline Oregon ………............... & 2.47 & 12.8 & 0 & 12.8 & 15.3 & 0 & 15.3 & 17.2 & 0 & 17.2 \\
\hline Pennsylvania .................... & 3.98 & 6,970 & 0 & 6,970 & 6,980 & 0 & 6,980 & 7,820 & 0 & 7,820 \\
\hline Rhode Island .................... & 0 & 2.40 & 290 & 293 & 2.40 & 290 & 293 & 2.69 & 326 & 328 \\
\hline South Carolina ............. & 5.83 & 5,700 & 0 & 5,700 & 5,710 & 0 & 5,710 & 6,400 & 0 & 6,400 \\
\hline South Dakota ................... & 1.23 & 4.01 & 0 & 4.01 & 5.24 & 0 & 5.24 & 5.87 & 0 & 5.87 \\
\hline Tennessee .......................... & 0 & 9,040 & 0 & 9,040 & 9,040 & 0 & 9,040 & 10,100 & 0 & 10,100 \\
\hline Texas .............................. & 60.2 & 9,760 & 3,440 & 13,200 & 9,820 & 3,440 & 13,300 & 11,000 & 3,860 & 14,900 \\
\hline 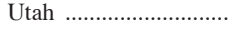 & 13.1 & 49.2 & 0 & 49.2 & 62.2 & 0 & 62.2 & 69.8 & 0 & 69.8 \\
\hline Vermont .......................... & .66 & 355 & 0 & 355 & 355 & 0 & 355 & 398 & 0 & 398 \\
\hline Virginia ............................. & 1.50 & 3,850 & 3,580 & 7,430 & 3,850 & 3,580 & 7,430 & 4,310 & 4,020 & 8,330 \\
\hline 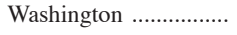 & .92 & 518 & 0 & 518 & 519 & 0 & 519 & 582 & 0 & 582 \\
\hline West Virginia ................ & 0 & 3,950 & 0 & 3,950 & 3,950 & 0 & 3,950 & 4,430 & 0 & 4,430 \\
\hline Wisconsin ....................... & 8.99 & 6,090 & 0 & 6,090 & 6,090 & 0 & 6,090 & 6,830 & 0 & 6,830 \\
\hline Wyoming ……................. & 1.13 & 242 & 0 & 242 & 243 & 0 & 243 & 273 & 0 & 273 \\
\hline Puerto Rico ...................... & 0 & 0 & 2,190 & 2,190 & 0 & 2,190 & 2,190 & 0 & 2,460 & 2,460 \\
\hline U.S. Virgin Islands .... & 0 & 0 & 136 & 136 & 0 & 136 & 136 & 0 & 153 & 153 \\
\hline TOTAL & 409 & 135,000 & 59,500 & 195,000 & 136,000 & 59,500 & 195,000 & 152,000 & 66,700 & 219,000 \\
\hline
\end{tabular}



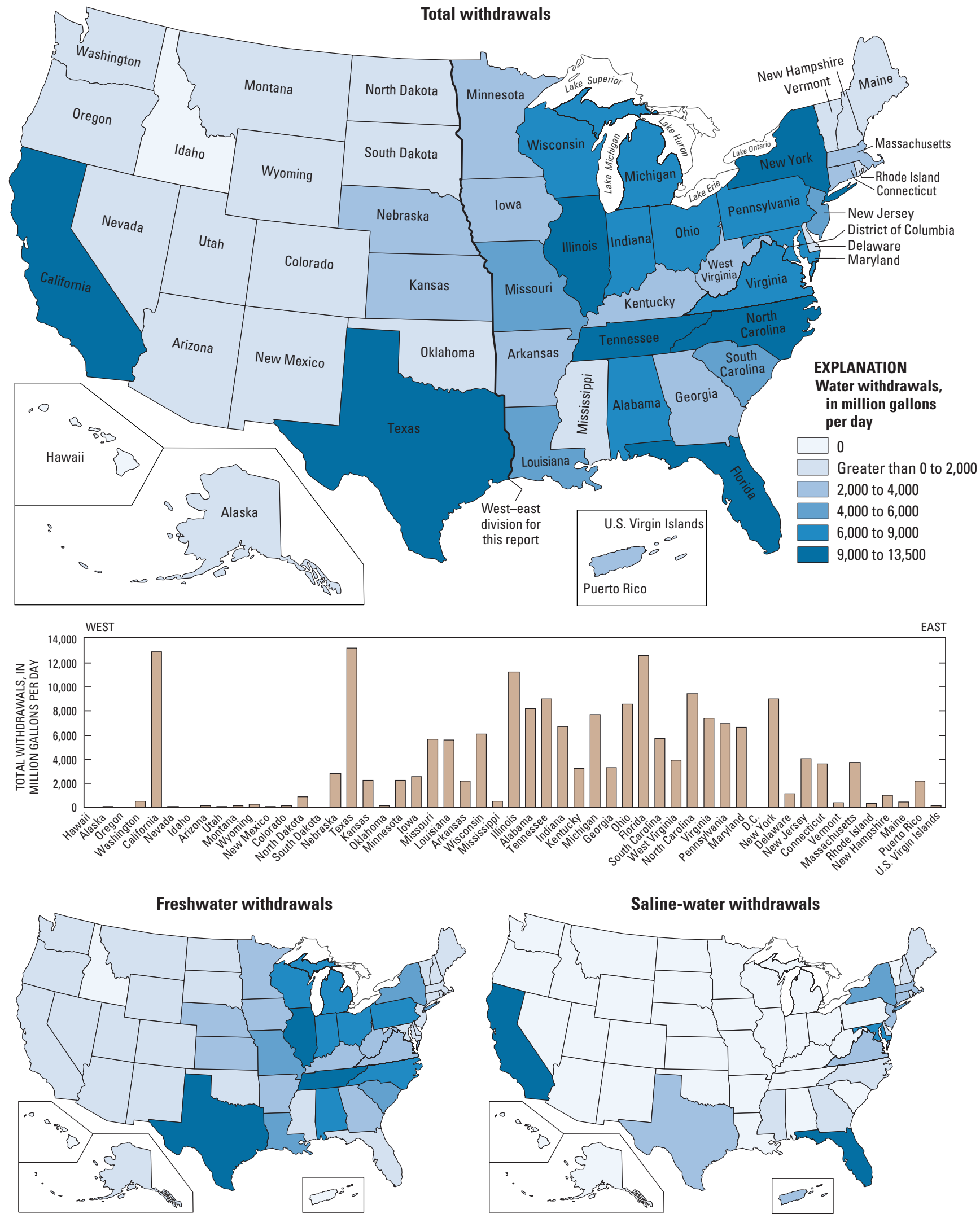

Figure 12. Thermoelectric-power withdrawals by water quality and State, 2000. 
Table 13. Thermoelectric-power water withdrawals by cooling type, 2000.

[Totals may not sum because of independent rounding. All values are in million gallons per day]

\begin{tabular}{|c|c|c|c|c|c|c|c|c|c|c|}
\hline \multirow{4}{*}{ STATE } & \multicolumn{3}{|c|}{$\begin{array}{l}\text { WITHDRAWALS FOR } \\
\text { ONCE-THROUGH COOLING }\end{array}$} & \multicolumn{7}{|c|}{ WITHDRAWALS FOR CLOSED-LOOP COOLING } \\
\hline & \multirow{2}{*}{\multicolumn{3}{|c|}{ Surface water }} & \multicolumn{4}{|c|}{ By source and type } & & & \\
\hline & & & & Ground water & & Irface we & & \multicolumn{3}{|c|}{ Total } \\
\hline & Fresh & Saline & Total & Fresh & Fresh & Saline & Total & Fresh & Saline & Total \\
\hline Alabama ......................... & 8,020 & 0 & 8,020 & 0 & 167 & 0 & 167 & 167 & 0 & 167 \\
\hline Alaska ........................... & 28.9 & 0 & 28.9 & 4.65 & 0 & 0 & 0 & 4.65 & 0 & 4.65 \\
\hline 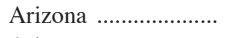 & 0 & 0 & 0 & 74.3 & 26.2 & 0 & 26.2 & 100 & 0 & 100 \\
\hline Arkansas ......................... & 1,690 & 0 & 1,690 & 2.92 & 478 & 0 & 478 & 481 & 0 & 481 \\
\hline California ..................... & 344 & 12,600 & 12,900 & 3.23 & 5.41 & 0 & 5.41 & 8.64 & 0 & 8.64 \\
\hline Colorado ........................ & 90.2 & 0 & 90.2 & 16.1 & 31.8 & 0 & 31.8 & 48.0 & 0 & 48.0 \\
\hline Connecticut ................ & 115 & 3,440 & 3,560 & .08 & 71.7 & 0 & 71.7 & 71.8 & 0 & 71.8 \\
\hline Delaware .......................... & 0 & 0 & 0 & .47 & 366 & 738 & 1,100 & 366 & 738 & 1,100 \\
\hline District of Columbia & 0 & 0 & 0 & 0 & 9.69 & 0 & 9.69 & 9.69 & 0 & 9.69 \\
\hline Florida ......................... & 559 & 11,800 & 12,400 & 29.5 & 69.8 & 150 & 219 & 99.3 & 150 & 249 \\
\hline Georgia ……................ & 2,800 & 61.7 & 2,860 & 1.03 & 444 & 0 & 444 & 445 & 0 & 445 \\
\hline Hawaii ........................... & 0 & 0 & 0 & 0 & 0 & 0 & 0 & 0 & 0 & 0 \\
\hline Idaho ............................. & 0 & 0 & 0 & 0 & 0 & 0 & 0 & 0 & 0 & 0 \\
\hline Illinois ........................... & 11,000 & 0 & 11,000 & 5.75 & 239 & 0 & 239 & 245 & 0 & 245 \\
\hline Indiana .......................... & 6,450 & 0 & 6,450 & 2.58 & 252 & 0 & 252 & 254 & 0 & 254 \\
\hline Iowa ……...................... & 2,510 & 0 & 2,510 & 11.9 & 15.6 & 0 & 15.6 & 27.6 & 0 & 27.6 \\
\hline Kansas ............................ & 2,210 & 0 & 2,210 & 14.9 & 29.1 & 0 & 29.1 & 44.0 & 0 & 44.0 \\
\hline Kentucky ...................... & 824 & 0 & 824 & 2.71 & 2,430 & 0 & 2,430 & 2,430 & 0 & 2,430 \\
\hline Louisiana .................... & 4,500 & 0 & 4,500 & 28.4 & 1,080 & 0 & 1,080 & 1,110 & 0 & 1,110 \\
\hline Maine ................................... & 90.8 & 295 & 385 & 4.92 & 17.2 & 0 & 17.2 & 22.2 & 0 & 22.2 \\
\hline Maryland ..................... & 377 & 5,670 & 6,050 & 1.80 & 0 & 589 & 589 & 1.80 & 589 & 591 \\
\hline Massachusetts ........... & 108 & 3,610 & 3,720 & 0 & .45 & 0 & .45 & .45 & 0 & .45 \\
\hline Michigan .......................... & 7,710 & 0 & 7,710 & 0 & 0 & 0 & 0 & 0 & 0 & 0 \\
\hline Minnesota ...................... & 1,330 & 0 & 1,330 & 4.17 & 935 & 0 & 935 & 939 & 0 & 939 \\
\hline Mississippi .................. & 307 & 148 & 456 & 43.5 & 11.2 & 0 & 11.2 & 54.7 & 0 & 54.7 \\
\hline Missouri ........................ & 5,200 & 0 & 5,200 & 12.2 & 422 & 0 & 422 & 434 & 0 & 434 \\
\hline Montana ........................ & 84.4 & 0 & 84.4 & 0 & 25.6 & 0 & 25.6 & 25.6 & 0 & 25.6 \\
\hline Nebraska ........................ & 2,390 & 0 & 2,390 & 6.87 & 418 & 0 & 418 & 424 & 0 & 424 \\
\hline Nevada ............................ & 0 & 0 & 0 & 12.0 & 24.7 & 0 & 24.7 & 36.7 & 0 & 36.7 \\
\hline New Hampshire ........ & 234 & 761 & 995 & .71 & 1.37 & 0 & 1.37 & 2.08 & 0 & 2.08 \\
\hline New Jersey ……............. & 648 & 3,330 & 3,980 & 2.24 & 0 & 57.6 & 57.6 & 2.24 & 57.6 & 59.9 \\
\hline New Mexico ............... & 0 & 0 & 0 & 11.4 & 45.0 & 0 & 45.0 & 56.4 & 0 & 56.4 \\
\hline New York ....................... & 4,040 & 5,010 & 9,050 & 0 & 0 & 0 & 0 & 0 & 0 & 0 \\
\hline North Carolina ........... & 7,850 & 1,620 & 9,470 & .09 & 0 & 0 & 0 & .09 & 0 & .09 \\
\hline North Dakota ............. & 887 & 0 & 887 & 0 & 14.5 & 0 & 14.5 & 14.5 & 0 & 14.5 \\
\hline Ohio ………...................... & 7,790 & 0 & 7,790 & 7.57 & 799 & 0 & 799 & 806 & 0 & 806 \\
\hline Oklahoma ………......... & 37.9 & 0 & 37.9 & 3.27 & 105 & 0 & 105 & 109 & 0 & 109 \\
\hline Oregon ……................... & 0 & 0 & 0 & 2.47 & 12.8 & 0 & 12.8 & 15.3 & 0 & 15.3 \\
\hline Pennsylvania .............. & 4,330 & 0 & 4,330 & 3.98 & 2,650 & 0 & 2,650 & 2,650 & 0 & 2,650 \\
\hline Rhode Island .................. & 0 & 290 & 290 & 0 & 2.40 & 0 & 2.40 & 2.40 & 0 & 2.40 \\
\hline South Carolina ........... & 3,860 & 0 & 3,860 & 5.83 & 1,850 & 0 & 1,850 & 1,850 & 0 & 1,850 \\
\hline South Dakota ............. & 0 & 0 & 0 & 1.23 & 4.01 & 0 & 4.01 & 5.24 & 0 & 5.24 \\
\hline Tennessee ..................... & 8,860 & 0 & 8,860 & 0 & 174 & 0 & 174 & 174 & 0 & 174 \\
\hline Texas ........................... & 6,990 & 3,440 & 10,400 & 60.2 & 2,770 & 0 & 2,770 & 2,830 & 0 & 2,830 \\
\hline Utah ........................ & 0 & 0 & 0 & 13.1 & 49.2 & 0 & 49.2 & 62.2 & 0 & 62.2 \\
\hline Vermont ........................ & 354 & 0 & 354 & .66 & .55 & 0 & .55 & 1.21 & 0 & 1.21 \\
\hline Virginia ............................ & 3,850 & 3,580 & 7,430 & 1.50 & 0 & 0 & 0 & 1.50 & 0 & 1.50 \\
\hline Washington ..................... & 444 & 0 & 444 & .92 & 74.2 & 0 & 74.2 & 75.1 & 0 & 75.1 \\
\hline West Virginia ............. & 3,790 & 0 & 3,790 & 0 & 163 & 0 & 163 & 163 & 0 & 163 \\
\hline Wisconsin ...................... & 6,090 & 0 & 6,090 & 8.99 & 0 & 0 & 0 & 8.99 & 0 & 8.99 \\
\hline Wyoming ...................... & 179 & 0 & 179 & 1.13 & 63.4 & 0 & 63.4 & 64.6 & 0 & 64.6 \\
\hline Puerto Rico ................... & 0 & 2,190 & 2,190 & 0 & 0 & 0 & 0 & 0 & 0 & 0 \\
\hline U.S. Virgin Islands .. & 0 & 136 & 136 & 0 & 0 & 0 & 0 & 0 & 0 & 0 \\
\hline TOTAL & 119,000 & 58,000 & 177,000 & 409 & 16,300 & 1,530 & 17,900 & 16,800 & 1,530 & 18,300 \\
\hline
\end{tabular}




\section{TRENDS IN WATER USE, 1950-2000}

The USGS first conducted the water-use compilations for 1950 and has published them every 5 years since. Groupings of categories and data elements have changed through the years. Water-use categories were combined in some compilations and were published as separate categories in others. Summaries of withdrawal estimates from 1950 to 2000 are shown in table 14 and shown graphically in figures 13 and 14. Self-supplied domestic withdrawals are shown separately from livestock and aquaculture withdrawals in table 14, although these uses were combined in the rural domestic and livestock category in previous wateruse Circulars and are shown together in figure 14.

The livestock category represents water use for farm animals (or stock) from 1950 through 1980. The livestock and aquaculture withdrawal estimates in table 14 include fish farms from 1985 through 2000. For 2000 , this category includes water use for stock, fish farms and fish hatcheries in table 14.

The industrial category also has been treated differently in various compilation years. The industrial water-use data for 1960 through 1980 represented withdrawals for facilities traditionally considered industrial as well as water use by commercial and mining facilities, fish farms, and fish hatcheries.

This combined category was called "other industrial use" to distinguish these uses from thermoelectricpower generation water use. For the years 1985, 1990 and 1995, wateruse data for industrial, commercial, and mining uses were published as separate categories. For 2000, industrial and mining withdrawals were compiled and published as separate categories, and commercial use was not compiled nationally. In table 14, commercial and mining withdrawals have been added to the industrial withdrawals for 1985 through 1995 so that the data are comparable to the "other industrial use" category in previous water-use Circulars. The "other industrial use" category cannot be calculated for 2000 because an estimate of commercial withdrawals was not compiled for 2000.

Estimates of withdrawals are summarized in table 14 at 5-year intervals from 1950 through 2000. The percentage change in withdrawals for each category (or category combination) is shown between 1995 and 2000. Estimates of total, ground-water, and surface-water withdrawals and total population are shown in figure 13. Figure 14 illustrates total freshwater and saline-water withdrawals by category.

Estimates in table 14 and figure 13 show total withdrawals increased steadily from 1950 to 1980 , declined more than 9 percent from 1980 to 1985 , and have varied less than 3 percent between the 5 -year intervals since 1985 . Total withdrawals peaked during 1980, although total U.S. population has increased steadily since 1950. Estimates of water use peaked during 1980 because of large industrial, irrigation, and thermoelectric-power withdrawals. Total withdrawals for 2000 were similar to the 1990 total withdrawals, although population had increased 13 percent since 1990

Total withdrawals have remained about 80 percent surface water and 20 percent ground water during the 50-year period. The portion of surface-water withdrawals that was saline increased from 7 percent for 1950 to 20 percent for 1975 and has remained about 20 percent since. The percentage of ground water that was saline never exceeded about 2 percent. The percentage of total withdrawals that was saline water increased from a minor amount in 1950 to as much as 17 percent during 1975 and 1990.

Estimated withdrawals for public supply increased continually since 1950 (fig. 14) along with population served by public suppliers. Publicsupply withdrawals more than tripled during this 50-year period and increased about 8 percent from 1995 to 2000 . The percentage of population served by public suppliers increased from 62 percent for 1950 to 85 percent for 2000 . Public-supply withdrawals represented about 8 percent of total withdrawals for 1950 and about 11 percent for 2000. The percentage of ground-water use for public supply increased from 26 percent for 1950 to 40 percent for 1985 and has remained at slightly less than 40 percent since.

Estimated withdrawals for self-supplied domestic use increased by 71 percent between 1950 and 2000. The selfsupplied domestic population was 57.5 million people for 1950 , or 38 percent of the total population. For 2000, 43.5 million people, or 15 percent of the total population, were self-supplied. 
Table 14. Trends in estimated water use in the United States, 1950-2000.

[Data for 1995 and earlier from Solley and others (1998). The water-use data are in billion gallons per day (thousand million gallons per day) and are rounded to two significant figures for 1950-80, and to three significant figures for 1985-2000; percentage change is calculated from unrounded numbers. ,- not available]

\begin{tabular}{|c|c|c|c|c|c|c|c|c|c|c|c|c|}
\hline & \multicolumn{11}{|c|}{ YEAR } & \multirow{2}{*}{$\begin{array}{l}\text { PERCENTAGE } \\
\text { CHANGE } \\
1995-2000\end{array}$} \\
\hline & ${ }^{1} 1950$ & ${ }^{2} 1955$ & ${ }^{3} 1960$ & ${ }^{4} 1965$ & ${ }^{4} 1970$ & ${ }^{3} 1975$ & ${ }^{3} 1980$ & ${ }^{3} 1985$ & ${ }^{3} 1990$ & ${ }^{3} 1995$ & ${ }^{3} 2000$ & \\
\hline Population, in millions & 150.7 & 164.0 & 179.3 & 193.8 & 205.9 & 216.4 & 229.6 & 242.4 & 252.3 & 267.1 & 285.3 & +7 \\
\hline \multicolumn{13}{|l|}{ Offstream use: } \\
\hline Total withdrawals & 180 & 240 & 270 & 310 & 370 & 420 & 440 & 399 & 408 & 402 & 408 & +2 \\
\hline Public supply & 14 & 17 & 21 & 24 & 27 & 29 & 34 & 36.5 & 38.5 & 40.2 & 43.2 & +8 \\
\hline \multicolumn{13}{|l|}{ Rural domestic and livestock: } \\
\hline Self-supplied domestic & 2.1 & 2.1 & 2.0 & 2.3 & 2.6 & 2.8 & 3.4 & 3.32 & 3.39 & 3.39 & 3.59 & +6 \\
\hline Livestock and aquaculture & 1.5 & 1.5 & 1.6 & 1.7 & 1.9 & 2.1 & 2.2 & ${ }^{5} 4.47$ & 4.50 & 5.49 & $\left({ }^{6}\right)$ & - \\
\hline Irrigation & 89 & 110 & 110 & 120 & 130 & 140 & 150 & 137 & 137 & 134 & 137 & +2 \\
\hline \multicolumn{13}{|l|}{ Industrial: } \\
\hline Thermoelectric-power use & 40 & 72 & 100 & 130 & 170 & 200 & 210 & 187 & 195 & 190 & 195 & +3 \\
\hline Other industrial use & 37 & 39 & 38 & 46 & 47 & 45 & 45 & 30.5 & 29.9 & 29.1 & $\left({ }^{7}\right)$ & - \\
\hline \multicolumn{13}{|l|}{ Source of water: } \\
\hline \multicolumn{13}{|l|}{ Ground: } \\
\hline Fresh & 34 & 47 & 50 & 60 & 68 & 82 & 83 & 73.2 & 79.4 & 76.4 & 83.3 & +9 \\
\hline Saline & $\left({ }^{8}\right)$ & .6 & .4 & .5 & 1.0 & 1.0 & .9 & .65 & 1.22 & 1.11 & 1.26 & +14 \\
\hline \multicolumn{13}{|l|}{ Surface: } \\
\hline Fresh & 140 & 180 & 190 & 210 & 250 & 260 & 290 & 265 & 259 & 264 & 262 & -1 \\
\hline Saline & 10 & 18 & 31 & 43 & 53 & 69 & 71 & 59.6 & 68.2 & 59.7 & 61.0 & +2 \\
\hline $\begin{array}{l}148 \text { States and District of Columb } \\
248 \text { States and District of Columb } \\
{ }^{3} 50 \text { States and District of Columb } \\
{ }^{4} 50 \text { States and District of Columb }\end{array}$ & $\begin{array}{l}\text {, Puerto } \\
\text {, and Pu }\end{array}$ & $\begin{array}{l}\mathrm{co} \text {, and } \mathrm{L} \\
\text { Rico }\end{array}$ & Virgir & & & $\begin{array}{l}{ }^{5} \text { From } \\
{ }^{6} \text { Data } n \\
{ }^{7} \text { Comm } \\
{ }^{8} \text { Data n }\end{array}$ & $\begin{array}{l}5 \text { to pres } \\
\text { available } \\
\text { ial use n } \\
\text { available }\end{array}$ & $\begin{array}{l}\text { this cate } \\
\text { r all State } \\
\text { available; }\end{array}$ & $\begin{array}{l}\text { ry includ } \\
\text { partial to } \\
\text { idustrial }\end{array}$ & $\begin{array}{l}\text { water us } \\
1 \text { was } 5.4 \mathrm{C} \\
\text { d mining }\end{array}$ & or fish fa & \\
\hline
\end{tabular}

Withdrawals for livestock and aquaculture use increased from 1.5 Bgal/d during 1950 to $5.49 \mathrm{Bgal} / \mathrm{d}$ during 1995 . The use for these categories during 2000 was $5.46 \mathrm{Bgal} / \mathrm{d}$. The livestock and aquaculture estimate includes fish farms from 1985 through 2000. The water-use estimate for 2000 also includes fish hatcheries. Livestock water-use estimates for 2000 were not required from all States.

Since 1950, irrigation has represented about 65 percent of total withdrawals, excluding those for thermoelectric power. Withdrawals for irrigation increased by more than 68 percent from 1950 to 1980 (from 89 to $150 \mathrm{Bgal} / \mathrm{d}$ ). Withdrawals have decreased since 1980 and have stabilized at between 134 and $137 \mathrm{Bgal} / \mathrm{d}$ between 1985 and 2000. Depending on the geographic area of the United States, this overall decrease can be attributed to climate, crop type, advances in irrigation efficiency, and higher energy costs.

Surface water historically has been the primary source for irrigation, although data show an increasing usage of ground water since 1950. During 1950, 77 percent of all irrigation withdrawals were surface water, most of which was used in the western States. By 2000, surface-water withdrawals comprised only 58 percent of the total. Ground-water withdrawals for irrigation during 2000 were more than three times larger than during 1950. Most of this increase occurred from 1965 through 1980.

The total number of acres irrigated in the United States steadily increased from 25,000 thousand acres for 1950 to 58,000 thousand acres for 1980 . The estimated number of acres irrigated remained relatively constant from 1980 to 1995 , and then increased to 61,900 thousand acres during 2000. The increase in irrigated acreage during the 1960s and 1970s was related to the expansion of irrigation in the western States. The number of acres irrigated in 2000 increased in some States in response to drought.

Since 1985, when USGS first collected data on irrigated acres by system type, more acres were irrigated using sprinkler and microirrigation systems than were irrigated with flood systems. The proportion of total acres irrigated using 


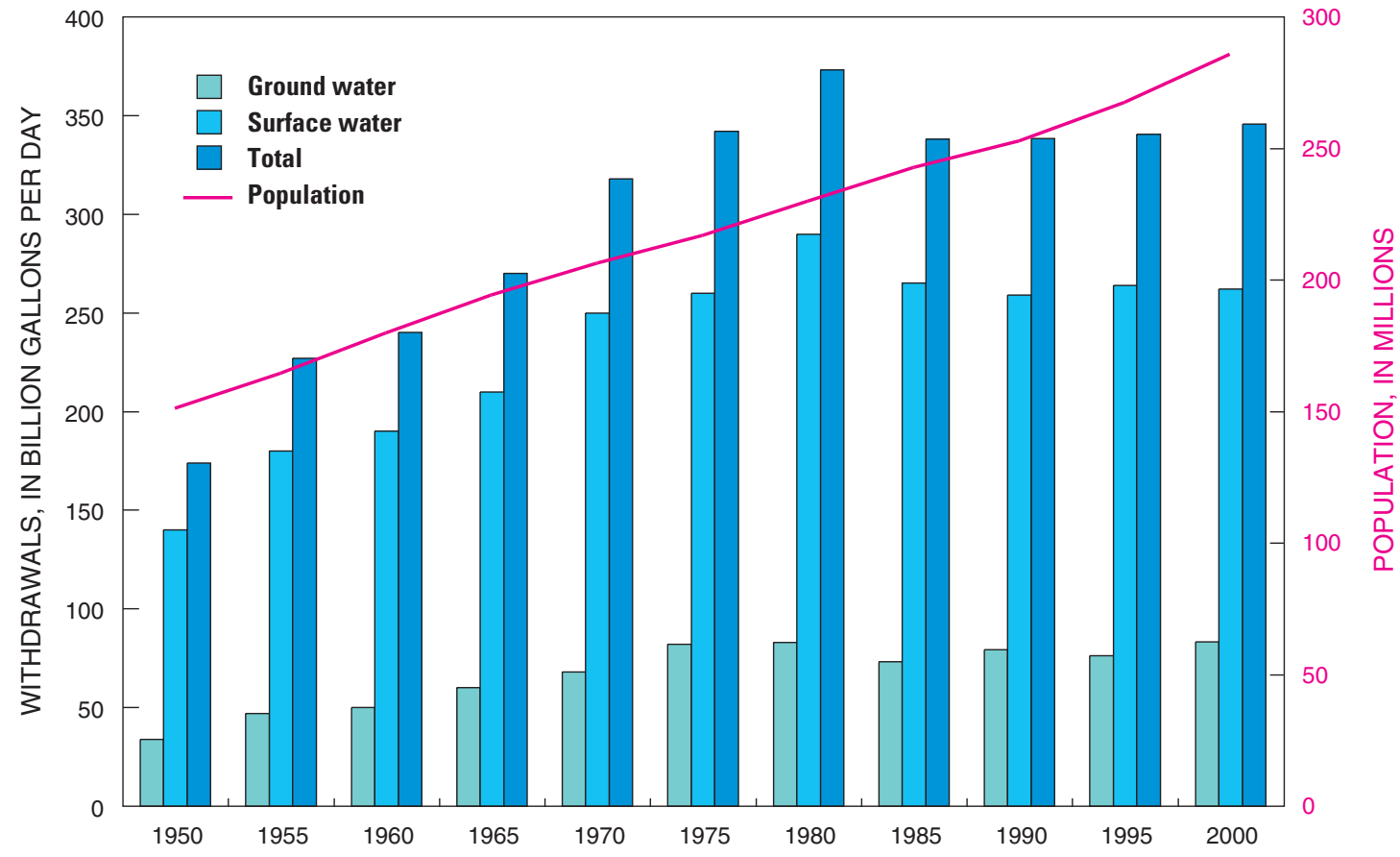

Figure 13. Trends in population and freshwater withdrawals by source, 1950-2000.

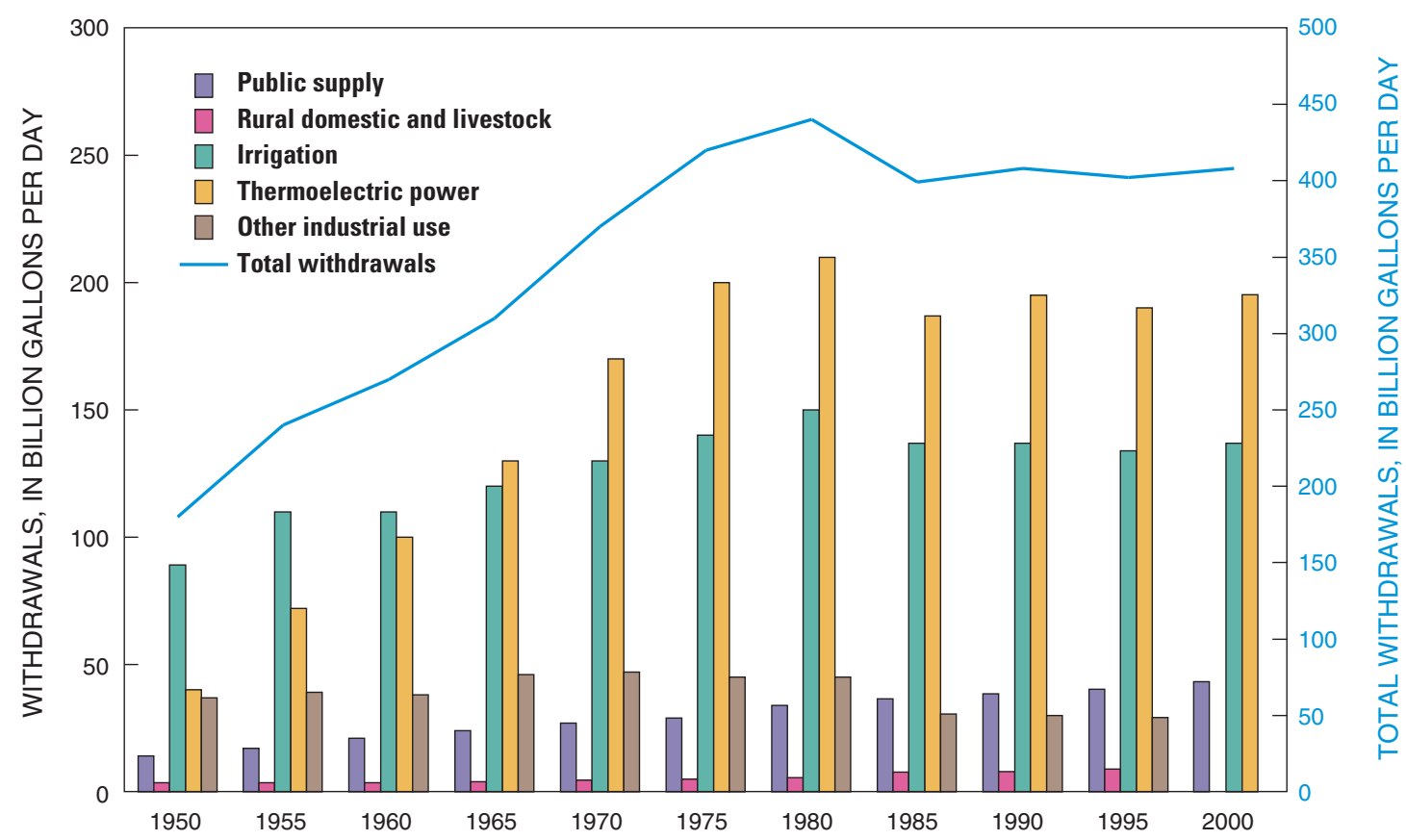

Figure 14. Trends in total water withdrawals by water-use category, 1950-2000. (Total withdrawals for rural domestic and livestock and for "other industrial use" are not available for 2000.) 
sprinkler and microirrigation systems increased from less than 40 percent in 1985 to 52 percent for 2000 . The average irrigation application rate declined about 30 percent, from 3.55 acre-feet per acre during 1950 to 2.48 acre-feet per acre during 2000. The largest declines in application rates occurred after 1980.

Thermoelectric power has been the category with the largest water withdrawals since 1965, and for 2000 comprised 48 percent of total withdrawals. The largest total and fresh and saline surface-water withdrawals were during 1980. Withdrawals by thermoelectric-power plants increased from $40 \mathrm{Bgal} / \mathrm{d}$ during 1950 to $210 \mathrm{Bgal} / \mathrm{d}$ during 1980 . Withdrawals for thermoelectric power declined and then stabilized since 1980; the total withdrawal of $195 \mathrm{Bgal} / \mathrm{d}$ for 2000 is the same as the total withdrawal for 1990.

Thermoelectric-power water withdrawals primarily were affected by the Federal legislation that required stricter water-quality standards for return flow and by limited water supplies in some areas of the United States (U.S. Congress, Amendments to the Federal Pollution Control Act of 1972 and 1977; Micheletti and Burns, 2002). Consequently, since the 1970s, power plants increasingly were built with or converted to closed-loop cooling systems or air-cooled systems instead of using once-through cooling systems. By 2000, an alternative to once-through cooling was used in about 60 percent of the installed steam-generation capacity in the power plants (Bozek, 2002).

Use of recirculated water for cooling in a closed-loop system reduces the water requirement at the power plant, resulting in reduced water withdrawals. The increasing influence over time of these technologies that require less water can be observed in the historical USGS water-use record. The trend showing the increase, decline, then stabilization of water withdrawals for thermoelectric power from 1950 to 2000 occurred as net electricity generated increased almost 15 -fold to 3,450 billion kilowatt-hours ( $\mathrm{kWh}$ ) during this same period (U.S. Department of Energy, Energy Information Administration, 2003c). Overall, significantly less water was required to generate a kilowatt-hour of electricity for 2000 . The average gallons of water used to produce one kilowatt-hour ( $(\mathrm{gal} / \mathrm{kWh})$ decreased from $63 \mathrm{gal} / \mathrm{kWh}$ during 1950 to $21 \mathrm{gal} / \mathrm{kWh}$ during 2000 (Solley and others, 1998).

The industrial category has been treated differently in various compilation years. The industrial water-use data for 1960 through 1980 represented use for facilities traditionally considered as industrial as well as water use for commercial and mining facilities, fish farms, and fish hatcheries. For the years 1985, 1990, and 1995, water-use data were published as the separate categories of industrial, commercial, and mining. For 2000, withdrawal data were compiled for industrial and mining uses but not commercial use. In table 14, commercial and mining withdrawals were added to the industrial withdrawals to correspond to the "other industrial use" category of previous water-use Circulars. Because commercial data were not available for 2000 , a percentage change for 1995 to 2000 cannot be calculated for this combined category.

Withdrawals for "other industrial uses" were between 37 and $39 \mathrm{Bgal} / \mathrm{d}$ between 1950 and 1960, and then increased to the range of 45 and $47 \mathrm{Bgal} / \mathrm{d}$ for the years reported from 1965 to 1980 . Withdrawals declined by about 32 percent to $30 \mathrm{Bgal} / \mathrm{d}$ between 1980 and 1985; the decline continued to about $29 \mathrm{Bgal} / \mathrm{d}$ in 1995. Because data were not classified separately from 1955 to 1980 , it is not known how much of the "other industrial use" was attributable to commercial and mining withdrawals. However, it is evident that by 2000 the "other industrial use" category was at its lowest level since data were first reported for 1950. For the industrial withdrawals not to be at the lowest level since 1950, commercial water use, which was not reported for 2000, would have had to more than double between 1995 and 2000. That scenario for commercial water use is improbable.

Because withdrawal data were compiled independently for the industrial category for 1985 through 2000, the changes in the industrial category may be compared for those years. Total industrial withdrawals decreased by 24 percent from about $26 \mathrm{Bgal} / \mathrm{d}$ during 1985 to less than $20 \mathrm{Bgal} / \mathrm{d}$ during 2000 . Ground water provided 15 percent of the total industrial withdrawals during 1985 and 18 percent of the total during 1990, 1995, and 2000. Almost all of the ground water withdrawn for industrial uses was freshwater. The percentage of surface water that is fresh has increased from about 84 percent during 1985 to about 92 percent during 2000.

Two factors have affected the industrial water-use withdrawals. Passage of the Amendments to the Federal Pollution Control Act of 1972 and 1977 required stricter water-quality standards for water discharges, which in turn, encouraged conservation, greater efficiency, and lower water-using technologies. Decline in the number of manufacturing facilities during more recent years also has reduced industrial withdrawals. Employment in two of the major waterusing industries declined from 1985 to 2000 . Employment in the petroleum and coal products industries declined by 26 percent, and employment in the primary metal industries declined by 13 percent (U.S. Department of Commerce, Bureau of Economic Analysis, 2003). 


\section{References Cited}

Bozek, C.R., 2002, A towering challenge: Electrical Perspectives, v. 27, no. 1, January/February 2002: accessed December 18, 2003, at http://www.eei.org/magazine/editorial_ content/nonav_stories/2002-01-01-tower.htm

Hobbs, Frank, and Stoops, Nicole, November 2002, U.S. Department of Commerce, U.S. Census Bureau, Census 2000 Special Reports, Series CENSR-4, Demographic Trends in the 20th Century: U.S. Printing Office, Washington D.C., p. 143, accessed December 18, 2003, at http://www.census.gov/prod/2002pubs/censr-4.pdf

MacKichan, K.A., 1951, Estimated water use in the United States, 1950-U.S. Geological Survey Circular 115, 13 p.

MacKichan, K.A., 1957, Estimated water use in the United States, 1955: U.S. Geological Survey Circular 398, 18 p.

MacKichan, K.A., and Kammerer, J.C., 1961, Estimated use of water in the United States, 1960: U.S. Geological Survey Circular 456, $26 \mathrm{p}$.

Michelletti, W.C., and Burns, J.M., 2002, Emerging issues and needs in power plant cooling systems in Proceedings of the Workshop on "Electric Utilities and Water: Emerging Issues and R\&D Needs": Pittsburgh, Pennsylvania, July 23-24, 2002, accessed December 1, 2003, at http://www.netl.doe.gov/publications/proceedings 102/EU\&W/Micheletti_JMB.PDF

Murray, C.R., 1968, Estimated use of water in the United States, 1965: U.S. Geological Survey Circular 556, 53 p.

Murray, C.R., and Reeves, E.B., 1972, Estimated use of water in the United States, 1970: U.S. Geological Survey Circular $676,37 \mathrm{p}$.

Murray, C.R., and Reeves, E.B., 1977, Estimated use of water in the United States, 1975: U.S. Geological Survey Circular $765,37 \mathrm{p}$.

National Oceanic and Atmospheric Administration, 2001, Climate of 2000 - Annual review, U.S. Summary: National Climatic Data Center, accessed December 18, 2003, at http://www.ncdc.noaa.gov/oa/climate/research/2000/ ann/us_summary.html

Office of Management and Budget, 1987, Standard industrial classification manual: [Washington, D.C.], 705 p.

Solley, W.B., Chase, E.B., and Mann, W.B., IV, 1983, Estimated use of water in the United States in 1980: U.S. Geological Survey Circular 1001, 56 p.

Solley, W.B., Merk, C.F., and Pierce, R.R., 1988, Estimated use of water in the United States in 1985: U.S. Geological Survey Circular 1004, 82 p.
Solley, W.B., Pierce, R.R., and Perlman, H.A., 1993, Estimated use of water in the United States in 1990: U.S. Geological Survey Circular 1081, 76 p.

Solley, W.B., Pierce, R.R., and Perlman, H.A., 1998, Estimated use of water in the United States in 1995: U.S. Geological Survey Circular 1200, 71 p.

U.S. Congress, 1972, Amendments to the Federal Water Pollution Control Act of 1972, Public Law 92-500, Title 33, Chapter 26, subchapter 111, Section 1326 (a) and 1326 (b), accessed January 22, 2004, at http://www4.law.cornell.edu/ uscode/33/1326.html

U.S. Congress, 1977, Clean Water Act of 1977, Public Law 95-217, Title 33, Chapter 26, Subchapter 1, Section 1251, accessed January 22, 2004, at http://www4.law.cornell.edu/ uscode/33/1251.html

U.S. Department of Agriculture, National Agricultural Statistics Service, 1999, Ranking of States and Counties: 1997 Census of Agriculture, vol. 2, subject series p. 2, 110 p., accessed December 18, 2003, at http://www.nass.usda.gov/ census/census97/rankings/rankings.htm

U.S. Department of Agriculture, National Agricultural Statistics Service, 2000, Drought and Fire Survey 2000, Montana's 2000 Drought/Fire Survey Results: press release, accessed June 25, 2003, at http://www.nass.usda.gov/mt/ pressrls/misc/firesurv.htm

U.S. Department of Commerce, Bureau of Economic Analysis, 2003, report SA25, accessed July 11, 2003, at http: $/ / w w w . b e a . d o c . g o v /$ bea/regional/spi/drill.cfm?table $=S A 25 \&$ $l c=400 \&$ years $=2000,1995,1990,1985,1980,1975,1970,196$ 5,1960\&format $=h t m$

U.S. Department of Commerce, U.S. Census Bureau, 2000, Census 2000 Summary File 1 (SF 1) 100-Percent Data, accessed December 18, 2003, at http://factfinder.census.gov/ servlet/DTGeoSearchByListServlet? ds_name $=$ DEC_2000_ SF1_U\&_lang=en\&_ts=90149465759

U.S. Department of Energy, Energy Information Administration, 2003a, Annual steam-electric plant operation and design data, accessed October 27, 2003, at http: //www.eia.doe.gov/cneaf/electricity/page/eia767.html

U.S. Department of Energy, Energy Information Administration, 2003b, Monthly utility power plant database, accessed October 27, 2003, at http://www.eia.doe.gov/cneaf/ electricity/page/eia906u.html

U.S. Department of Energy, Energy Information Administration, 2003c, Electricity, chap. 8 of Annual Energy Review 2002: U.S. Department of Energy, DOE/EIA-0384 (2002), p. 221-238, accessed October 27, 2003, at http: //www.eia.doe.gov/emeu/aer/pdf/pages/sec8.pdf 


\section{GlOSSARY}

Water-use terminology has changed in this series of water-use Circulars prepared at 5-year intervals. The term water use as initially used for 1950 in the USGS water-use Circulars meant withdrawals of water; in the report for 1960, the term was redefined to include consumptive use of water as well as withdrawals. With the beginning of the USGS National Water Use Information Program in 1978, the term was again redefined to include return flow and offstream and instream uses. In the report for 1985, the term was redefined to include withdrawals plus deliveries from public suppliers. In the water-use Circular for 2000, water use is defined as initially used in 1950 as withdrawals of water. The following terms are referenced in the text and are part of the water-use Circular series.

animal-specialties water use-water use associated with the production of fish in captivity, except for fish hatcheries, and the raising of horses and such fur-bearing animals as rabbits and pets. Animal-specialties water use estimates were included in some previous water-use Circulars, but were combined with the livestock categories or aquaculture categories for 2000. See also aquaculture water use, fish-farm water use, livestock water use, and rural water use.

aquaculture water use-water use associated with the farming of organisms that live in water-such as finfish and shellfish - and offstream water use associated with fish hatcheries. See also fish-farm water use, fish-hatchery water use, animal-specialties water use, and livestock water use.

closed-loop cooling system-cooling systems where water is withdrawn from a source, circulated through heat exchangers, then cooled, and recycled. Subsequent water withdrawals are used to replace water lost to evaporation, blowdown, drift, and leakage and, accordingly, results in a much smaller return flow than once-through cooling. See also cooling system, cooling-system type, industrial water use, and thermoelectricpower water use.

commercial water use - water for motels, hotels, restaurants, office buildings, other commercial facilities, military and nonmilitary institutions - and for 1990 and 1995, water for offstream fish hatcheries. Water may be obtained from a public-supply system or may be self-supplied. Commercial water-use estimates were included in some previous wateruse Circulars but were omitted for 2000. See also fishhatchery water use, public-supply water use, public-supply deliveries, and self-supplied water use.

consumptive use - the part of water withdrawn that is evaporated, transpired, incorporated into products or crops, consumed by humans or livestock, or otherwise removed from the immediate water environment. Consumptive-use estimates were included in some previous water-use Circulars but were omitted for 2000. Also referred to as water consumed. conveyance loss - water that is lost in transit from a pipe, canal, conduit, or ditch by leakage or evaporation. Generally, the water is not available for further use; however, leakage from an irrigation ditch, for example, may percolate to a ground-water source and be available for further use. Conveyance-loss estimates were included in some previous water-use Circulars but were omitted for 2000. See also irrigation water use.

cooling system - an equipment system that provides water for cooling purposes, such as to condensers at power plants or at factories, and includes water intakes and outlets; cooling towers; and ponds, pumps, and pipes. See also cooling-system type, industrial water use, and thermoelectric-power water use.

cooling-system type-See closed-loop cooling system and once-through cooling system.

domestic water use - water used for all such indoor household purposes as drinking, food preparation, bathing, washing clothes and dishes, flushing toilets, and such outdoor purposes as watering lawns and gardens. The term used in previous water-use Circulars described the combined public supply deliveries to residential users and self-supplied domestic withdrawals. For 2000, domestic water use refers only to self-supplied domestic withdrawals. See also public-supply deliveries, public-supply water use, rural water use, and selfsupplied water use.

fish-farm water use - water used for the production of finfish and shellfish under controlled feeding, sanitation, and harvesting procedures for commercial purposes. Water use by fish farms is classified in the aquaculture category. See also animal-specialties water use, aquaculture water use, and fishhatchery water use.

fish-hatchery water use - water used for raising fish for later release and in association with the operation of fish hatcheries or fishing preserves. Fish-hatchery water use is classified in the aquaculture category. See also aquaculture water use, commercial water use, and fish-farm water use.

freshwater-water that contains less than 1,000 milligrams per liter $(\mathrm{mg} / \mathrm{L})$ of dissolved solids; generally, more than $500 \mathrm{mg} / \mathrm{L}$ of dissolved solids is undesirable for drinking and for many industrial uses. See also saline water.

industrial water use - water used for fabrication, processing, washing, and cooling, and includes such industries as chemical and allied products, food, mining, paper and allied products, petroleum refining, and steel. Term used in previous water-use Circulars to describe the combined public-supply deliveries to industrial users and self-supplied industrial withdrawals. For 2000, industrial water use refers only to self-supplied industrial withdrawals. See also cooling system, cooling-system type, mining water use, public-supply deliveries, public-supply water use, self-supplied water use, and thermoelectric-power water use. 
instream use - water that is used, but not withdrawn, from a surface-water source for such purposes as hydroelectricpower generation, navigation, water-quality improvement, fish propagation, and recreation. Instream water-use estimates for hydroelectric power were included in previous water-use Circulars but were omitted for 2000.

irrigation district - a cooperative, self-governing public corporation set up as a subdivision of the State government, with definite geographic boundaries, organized, and having taxing power to obtain and distribute water for irrigation of lands within the district; created under the authority of a State legislature with the consent of a designated fraction of the landowners or citizens. See also irrigation water use.

irrigation water use-water that is applied by an irrigation system to assist in the growing of crops and pastures or to maintain vegetative growth in recreational lands such as parks and golf courses. Irrigation includes water that is applied for pre-irrigation, frost protection, chemical application, weed control, field preparation, crop cooling, harvesting, dust suppression, the leaching of salts from the root zone, and water lost in conveyance. See also conveyance loss, microirrigation system, sprinkler irrigation system, and surface irrigation system.

livestock water use-water for livestock watering, feedlots, dairy operations, and other on-farm needs. Types of livestock include dairy cows and heifers, beef cattle and calves, sheep and lambs, goats, hogs and pigs, horses and poultry. See also animal-specialties water use, aquaculture water use, and rural water use.

microirrigation system - An irrigation system that wets only a discrete portion of the soil surface in the vicinity of the plant by means of applicators (orifices, emitters, porous tubing, perforated pipe, and so forth) operated under low pressure. The applicators can be placed on or below the surface of the ground or can be suspended from supports. See also irrigation water use, sprinkler irrigation system, and surface irrigation system.

mining water use - water used for the extraction of naturally occurring minerals including solids, such as coal, sand, gravel, and other ores; liquids, such as crude petroleum; and gases, such as natural gas. Also includes uses associated with quarrying, milling, and other preparations customarily done at the mine site or as part of a mining activity. Does not include water associated with dewatering of the aquifer that is not put to beneficial use. Also does not include water used in processing, such as smelting, refining petroleum, or slurry pipeline operations. These processing uses are included in industrial water use. See also industrial water use and self-supplied water use.

offstream use - water withdrawn or diverted from a groundwater or surface-water source for aquaculture, commercial, domestic self-supply, industrial, irrigation, livestock, mining, public supply, thermoelectric power, and other uses. See also entries for each of the previously mentioned uses. once-through cooling system-cooling systems in which the water is withdrawn from a source, circulated through heat exchangers, and then returned to a body of water at a higher temperature. Once-through cooling systems may be referred to as open-loop systems. See also cooling system, coolingsystem type, industrial water use, and thermoelectric-power water use.

public-supply deliveries-amount of water delivered from a public supplier to users for domestic, commercial, industrial, thermoelectric-power, or public-use purposes. Delivery estimates were included in some previous water-use Circulars but were omitted for 2000. See also commercial water use, domestic water use, industrial water use, public-supply water use, public water use, and thermoelectric-power water use.

public-supply water use - water withdrawn by public and private water suppliers that furnish water to at least 25 people or have a minimum of 15 connections. Public suppliers provide water for a variety of uses, such as domestic, commercial, industrial, thermoelectric power, and public water use. See also commercial water use, domestic water use, industrial water use, public-supply deliveries, public water use, and thermoelectric-power water use.

public water use - water supplied from a public supplier and used for such purposes as firefighting, street washing, flushing of water lines, and maintaining municipal parks and swimming pools. Generally, public-use water is not billed by the public supplier. See also public-supply deliveries and publicsupply water use.

reclaimed wastewater-wastewater treatment plant effluent that has been diverted for beneficial use before it reaches a natural waterway or aquifer. Term used in previous water-use Circulars. See also water use.

return flow - water that reaches a ground-water or surfacewater source after release from the point of use and thus becomes available for further use. Term used in previous water-use Circulars. See also water use.

rural water use - water used in suburban or farm areas for domestic and livestock needs. The water generally is selfsupplied, and includes domestic use, drinking water for livestock, and other uses, such as dairy sanitation, cleaning, and waste disposal. Term used in previous water-use Circulars. See also animal-specialties water use, domestic water use, livestock water use, and self-supplied water use.

saline water-water that contains $1,000 \mathrm{mg} / \mathrm{L}$ or more of dissolved solids. See also freshwater.

self-supplied water use - water withdrawn from a groundwater or surface-water source by a user rather than being obtained from a public supply.

sprinkler irrigation system - An irrigation system in which water is applied by means of perforated pipes or nozzles operated under pressure so as to form a spray pattern. See also irrigation water use, microirrigation system, and surface irrigation system. 
standard industrial classification (SIC) codes-four-digit codes established by the Office of Management and Budget, published in 1987, and used in the classification of establishments by type of activity in which they are engaged.

surface irrigation system -irrigation by means of flood, furrow, or gravity. Flood irrigation is the application of irrigation water in which the entire soil surface is covered by ponded water. Furrow is a partial surface-flooding method of irrigation normally used with clean-tilled crops in which water is applied in furrows or rows of sufficient capacity to contain the design irrigation stream. Gravity is an irrigation method in which water is not pumped, but flows in ditches or pipes and is distributed by gravity. See also irrigation water use, microirrigation system, and sprinkler irrigation system.

thermoelectric-power water use-water used in the process of generating electricity with steam-driven turbine generators. Term used in previous water-use Circulars to describe the combined public-supply deliveries to thermoelectric-power plants and self-supplied thermoelectric-power withdrawals. For 2000, thermoelectric-power water use refers only to selfsupplied thermoelectric-power withdrawals. See also cooling system, cooling-system type, public-supply water use, and self-supplied water use. wastewater-treatment return flow-term used in previous water-use Circulars to describe water returned to the hydrologic system by wastewater-treatment facilities. See also water use.

water use-(1) In a restrictive sense, the term refers to water that is withdrawn for a specific purpose, such as for public supply, domestic use, irrigation, thermoelectric-power cooling, or industrial processing. In previous water-use Circulars, water use for the domestic, commercial, industrial, and thermoelectric-power categories included both self-supplied withdrawals and deliveries from public supply. (2) More broadly, water use pertains to the interaction of humans with and influence on the hydrologic cycle, and includes elements such as water withdrawal, delivery, consumptive use, wastewater release, reclaimed wastewater, return flow, and instream use. See also offstream use and instream use.

watt-hour (Wh) - an electrical energy unit of measure equal to 1 watt of power supplied to, or taken from, an electric circuit steadily for 1 hour.

water withdrawal-water removed from the ground or diverted from a surface-water source for use. See also offstream use and self-supplied water use.

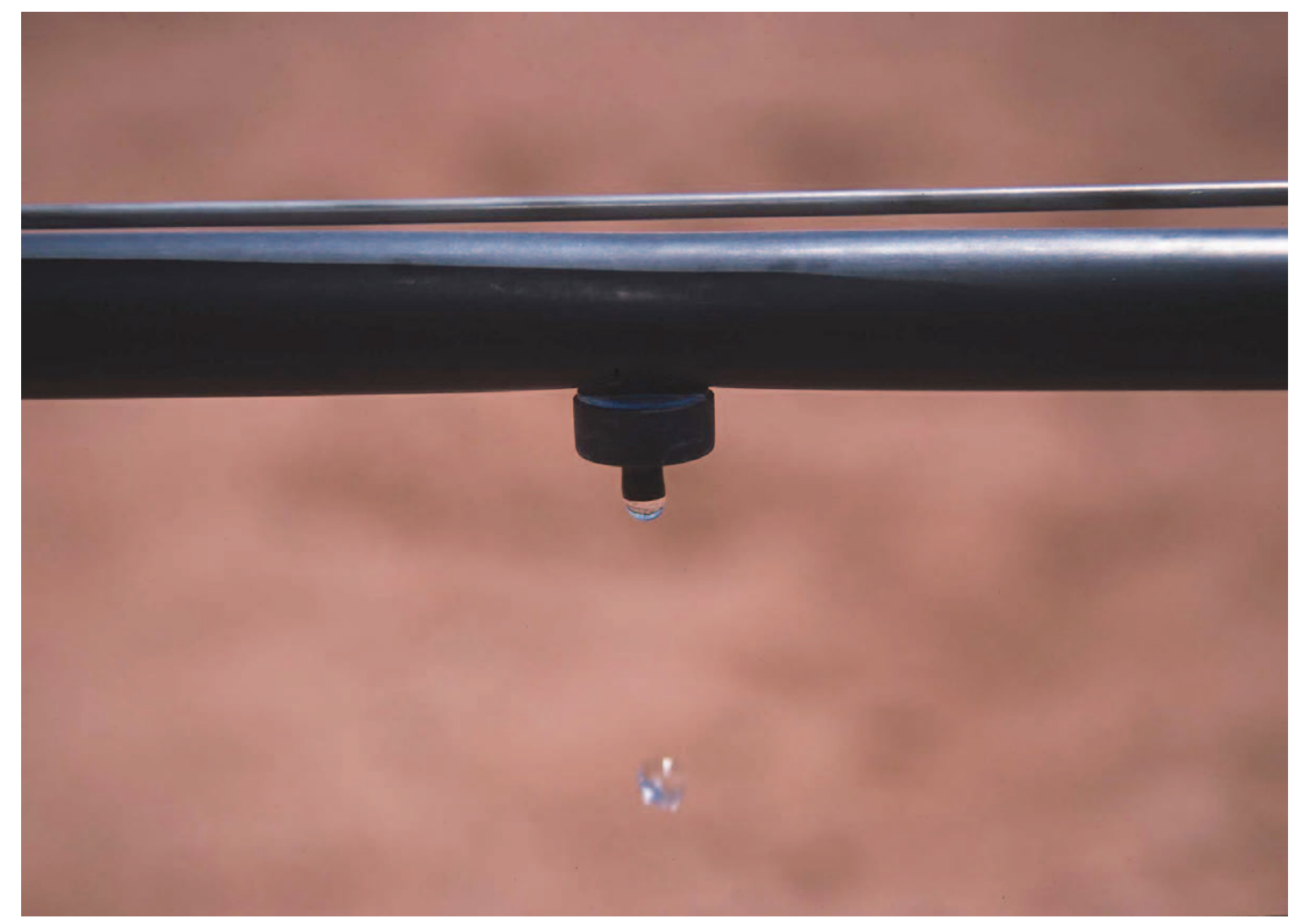

Drip irrigation emitter, New Mexico. (Photo courtesy of Jeff Vanuga, USDA Natural Resources Conservation Service.) 